\title{
Variational formulation and stability analysis of a three dimensional superelastic model for shape memory alloys
}

\author{
Roberto Alessi and Kim Pham
}

\begin{abstract}
This paper presents a variational framework for the three-dimensional macroscopic modeling of superelastic shape memory alloys in an isothermal setting. Phase transformation is accounted through a unique second order tensorial internal variable, acting as the transformation strain. Postulating the total strain energy density as the sum of a free energy and a dissipated energy, the model depends on two material scalar functions of the norm of the transformation strain and a material scalar constant. Appropriate calibration of these material functions allows to render a wide range of constitutive behaviours including stress-softening and stress-hardening. The quasi-static evolution problem of a domain is formulated in terms of two physical principles based on the total energy of the system: a stability criterion, which selects the local minima of the total energy, and an energy balance condition, which ensures the consistency of the evolution of the total energy with respect to the external loadings. The local phase transformation laws in terms of Kuhn-Tucker relations are deduced from the first-order stability condition and the energy balance condition.

The response of the model is illustrated with a numerical traction-torsion test performed on a thinwalled cylinder. Evolutions of homogeneous states are given for proportional and non-proportional loadings. Influence of the stress-hardening/softening properties on the evolution of the transformation domain is emphasized. Finally, in view of an identification process, the issue of stability of homogeneous states in a multi-dimensional setting is answered based on the study of second-order order derivative of the total energy. Explicit necessary and sufficient conditions of stability are provided.
\end{abstract}

Keywords: Shape Memory Alloys, Superelasticity, Stability criterion, Variational methods, Second-order stability condition, Stability of homogeneous states

\section{Contents}

1 Introduction 4

Notations ............................... 7

\begin{tabular}{|lll}
2 & Phenomenological approach & 7
\end{tabular}

2.1 Phenomenological material response . . . . . . . . . . . . . . . . . . . . . . . . . 7 


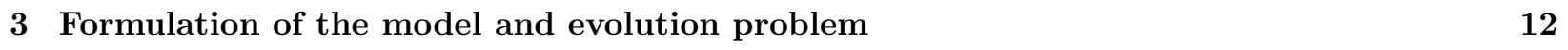

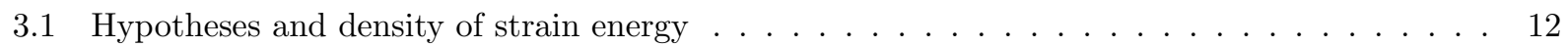

3.2 Evolution problem $\ldots \ldots \ldots \ldots \ldots \ldots \ldots \ldots \ldots$

4 Evolution laws: Phase transformation criteria and consistency conditions 14

$4.1 \quad$ Derivations of phase transformation criteria and consistency conditions . . . . . . . . . . 15

Mechanical Equilibrium . . . . . . . . . . . . . . . 16

Phase transformation criterion in $\Omega_{0} \ldots \ldots \ldots \ldots \ldots \ldots$. . . . . . . . . . . . .

Phase transformation criterion in $\Omega_{\mathrm{p}} \ldots \ldots \ldots \ldots \ldots \ldots \ldots$

Phase transformation criterion in $\Omega_{1}^{+} \cup \Omega_{1}^{-} \ldots \ldots \ldots \ldots \ldots \ldots$

From phase transformation criterion to first-order stability condition . . . . . . . . . 18

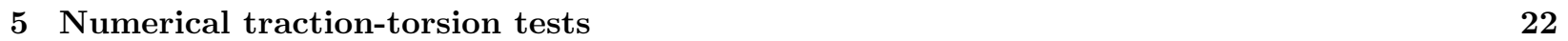

5.1 Non-Proportional loading material response $\ldots \ldots \ldots \ldots \ldots$. . . . . . . . . . . . . 24

5.2 Influence of $\mu(\|\mathbf{p}\|)$ on the transformation domain $\ldots \ldots \ldots \ldots \ldots$

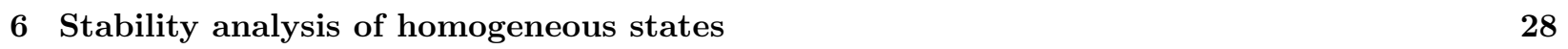

6.1 General statement . . . . . . . . . . . . . . . . . . . . . . . . . . . . 28

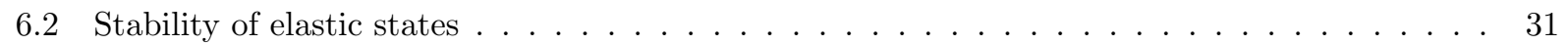

6.3 Stability of phase-transforming states $\ldots \ldots \ldots \ldots \ldots \ldots \ldots$

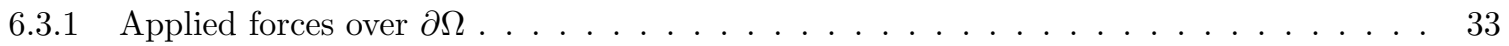

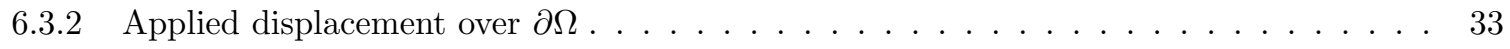

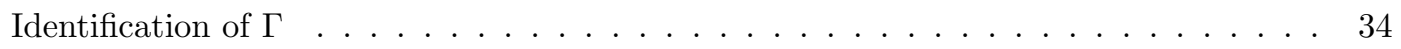

$6.3 .3 \quad$ Combined applied forces and displacements over $\partial \Omega . \ldots \ldots \ldots \ldots \ldots$

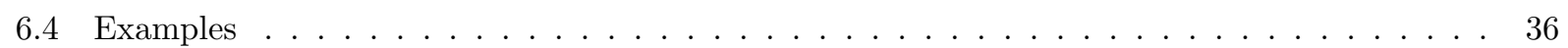

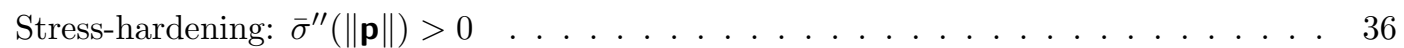

Stress-softening: $\bar{\sigma}^{\prime \prime}(\|\mathbf{p}\|)<0 \ldots \ldots \ldots \ldots \ldots \ldots \ldots$

\begin{tabular}{lll}
\hline 7 & Conclusions & 39 \\
\hline
\end{tabular} 


\begin{tabular}{|c|c|c|}
\hline \multicolumn{3}{|l|}{ Nomenclature } \\
\hline elastic Young's modulus & $\overline{\mathrm{p}}$ & cumulated phase transformation strain \\
\hline$\mu(y) \quad$ elastic shear modulus as material function & $N$ & dimension of the space \\
\hline $\begin{array}{ll}K & \text { elastic bulk modulus } \\
\bar{\sigma}_{0}, \Delta \sigma & \text { material constants }\end{array}$ & $\Omega$ & $\begin{array}{l}\text { reference configuration of the } N \text {-dimensional } \\
\text { body }\end{array}$ \\
\hline$\sigma_{\mathrm{Fw}}, \sigma_{\mathrm{Rv}}$ forward and reverse phase transformation & $W$ & total strain energy density \\
\hline stresses & $\mathcal{E}$ & total energy \\
\hline $\begin{array}{l}\bar{\sigma}(y) \quad \text { critical phase transformation stress as material } \\
\quad \text { function }\end{array}$ & $\mathcal{R}$ & Rayleigh ratio \\
\hline material function & $\mathcal{C}, \mathcal{C}_{0}$ & admissible spaces of displacement and displace- \\
\hline displacement & & ment rate fields \\
\hline$\varepsilon, \varepsilon \quad$ (total) strain & $\mathcal{Z}, \mathcal{Z}_{0}$ & admissible spaces of phase transformation \\
\hline stress & & strain and strain rate fields \\
\hline$\varepsilon^{D}, \sigma^{D}$ deviatoric strain and stress & $\overline{\mathcal{Z}}$ & admissible space of cumulated phase transfor- \\
\hline $\mathrm{p}, \mathbf{p} \quad$ phase transformation strain & & mation strain field \\
\hline
\end{tabular}




\section{Introduction}

Shape memory alloys (SMA) are materials which exhibits non-conventional thermo-mechanical properties, with two of the most remarkable being pseudoelasticity and shape memory effect. While the former refers to the capability of the material to regain the initial strain after a loading-unloading hysteretic cycle, the latter is related to the recovery of initial shape after occurrence of "apparently" permanent strains, simply by heating the material above a critical temperature. Both phenomena are associated to complex phase transitions at both micro- and macro-scale.

Due to these properties, polycrystalline SMA have been extensively studied with a great scientific impact and today are widely used for several advanced engineering applications like, among the others: biomedical devices, aerospace structures, mechanical components, MEMS as well as civil engineering structural elements or devices, (27] for a recent review of SMA about research, applications and opportunities). One of the key requirement of superelastic SMA is the size of its hysteresis loop. Indeed, a large hysteresis loop will lead to a substantial capability to dissipate energy. This is particularly important when SMA are used as dampers in civil engineering. Experimental investigations show that SMA with large hysteresis loop correspond to phase transformation occurring with a stress plateau and a localized phase transformation front [36]. However, a large number of loading cycles leads, as a result, to a smaller hysteresis loop with weakened dissipative properties and to an increasingly stress-hardening behaviour associated to a homogeneous phase transformation, 28].

The origin of macroscopic localization and stress plateau in SMA is related to an intrinsic stress-softening behaviour. Such kind of behaviour can be found in other type of dissipative phenomenon, such as damage, with similar consequences, e.g. macroscopic instabilities and non-homogeneous evolutions, [33. On the other hand, stress-hardening has a stabilizing effect and is usually associated to a unique diffuse response. Experimentally, such stress-softening behaviour leads to important issues in the identification of the material parameters of the model, [10, 20. Indeed, a classical tensile test on a SMA specimen provides direct information only if the response is homogeneous, so that the material behaviour can be read through the structural response, 24, 1. Stress-softening and macroscopic instabilities limit the use of such tensile test and non-conventional experimental setups are required to overcome such issues, [20]. The main idea in the recent experiments of Hallai and Kyriakides [17] is to force a homogeneous transformation, despite stress-softening, by bonding in parallel two strips, properly designed, of stress-hardening stainless steel with a softening SMA core strip. As a result, the global behaviour of the laminate exhibits a structural hardening response which is associated with a diffuse phase transformation. By subtracting the known and well-defined response of the stainless steel, one has access to the intrinsic behaviour of the SMA. The result is an up-and-down material response with a stress-hardening part only for a very short nominal strain range $(<1 \%)$ followed by a large stress-softening part $(\sim 5 \%)$. Due to residual plastic strains in the stainless steel strips, only the forward 
intrinsic phase transformation has been in this work extracted.

Another important feature of SMA is related to their macroscopic response under multi-axial loading. Specifically, although the literature is less dense than when it comes to uniaxial loading, several authors [37, 38, 25, 9, 19, 42, 14, 13, have studied the SMA behaviour in the multi-axial context with traction-torsion tests on thin-walled cylinders, where they highlighted a complex evolution of the specimen due to the reorientation of the martensite variants towards the direction of the mechanical loading. In particular, a dissymmetry in the material response has been observed with a stress-hardening homogeneous response in pure shear and a stress-softening response with localizations in simple tension. Unfortunately, few information is given on the transformation domain and its evolution in terms of shape and size except for the onset of the phase transformation. As in plasticity, the knowledge of such transformation domain is however fundamental for a rigorous construction of a phenomenological model. Another well established experimental evidence is that the mechanical response is almost independent with respect to hydrostatic pressure, hence with respect to the first invariant of the stress tensor. Indeed, as pointed out in the experiments in [16, pure hydrostatic pressure was unable to trigger the martensitic transformation at pressures of up to $700 \mathrm{MPa}$.

A consistent macroscopic model of SMA should then be able to account for all the aforementioned main characteristics, namely stress-softening, reorientation and incompressibility of the phase-transformation. Different classes of macroscopic models exist, either formulated phenomenologically or through a multi-scale approach. Usually two classes of constitutive models that account for superelasticity of SMA are distinguished. The first class of models correspond to constitutive behaviours derived from a micromechanical approach by means of homogenization techniques, [29, 23, 40, 41, 6, 7]. An advantage of these models is to give a direct access to the evolution of the microstructure during the macroscopic phase transformation and to relate the effective properties at the macroscopic scale to physical material parameters at the microscopic scale, 21]. However, these multi-scale approaches require usually too much computational efforts to be used for structural engineering calculations. In these latter cases it is more advisable to rely on macroscopic phenomenological models for which the phase transformation is described through internal variables [11, either scalar (for the martensitic volume fraction) or tensorial (for the martensite orientation strain tensor). Numerous models, based on the definition of a free-energy and a dissipation potential in the spirit of standard generalized materials, [18, have been proposed during the last decades including, for instance, shape memory effect (temperature-induced transformation), cycling effect, finite strains etc. etc., [5, 39, 34, 44, 45, 35]. Despite these models are able to describe a wide range of phenomena (superelasticity, thermo-mechanical coupling, shape memory effect, training), most of them are based on an intrinsic stress-hardening behaviour, hence leading to a homogeneous or diffuse phase transformation evolution.

Premises these considerations, we aim to provide in this paper a phenomenological isothermal 3D model capable of describing superelasticity in a general context where stress-softening, instabilities and martensite 
reorientation can occur. In order to deal with these behaviours, such model has to be formulated in a way to allow complex evolutions for which regularity in time and uniqueness of the response is not ensured. To this end, an ideal framework consists in well-established energetic approaches for the description of rateindependent processes, such as the Energetic Formulation mathematically formalized for standard generalized materials in 26. Once the total energy has been postulated, the quasi-static evolution problem is governed by two physical principles, a stability condition and an energy balance. While the former consists in selecting local (or global) minima of the total energy among admissible functional spaces the latter expresses the first law of thermodynamics by enforcing, with (absolute) continuity, the conservation of the total energy with respect to the loading parameter. In particular, the stability condition is an essential feature to deal with stress-softening behaviour for which non-uniqueness and multiple equilibrium states are the rule rather than the exception. Such kind of variational formulation has been fruitful applied in several contexts where stresssoftening occurs and complex patterns are observed, such as fracture [8], damage mechanics [31, plasticity [12], coupled damage-plasticity models [3] as well as phase transformation in the hardening context [4]. The model presented in this article can be considered as a 3D extension of [30, where a one dimensional local SMA model is formulated and investigated in the same spirit. Here, the model relies on a second-order, symmetric and deviatoric tensorial internal variable interpreted as a transformation strain, as in [39. Given the total energy of the system, the evolution laws will be variationally deduced from the stability criterion and energy balance condition. This approach gives also enough flexibility for future straightforward improvements of the model, such as dissymmetry in torsion-traction and phase transformation regularization, simply by modifying directly the total energy of the system.

The paper is organized as follows. In Section 2 , a phenomenological one-dimensional model is proposed as a prototype in order to highlight some important features and to justify the motivations to the energetic approach. Section 3 presents the formulation of the model and defines the evolution problem. First, state variables, with their associated functional spaces, are introduced and the total strain energy density specified. Then, the associated evolution problem is formulated by means of the stability criterion and energy balance. In Section 4, differential (first- and second-order) stability conditions are derived from the stability criterion by expanding in series the total energy. It is shown that first-order stability condition and time regularity of the energy balance are equivalent to a classical Karush-Kuhn-Tucker (KKT) system, consisting of a transformation domain, consistency condition and normality rule. Section 5 shows several numerical results of a thin-walled cylinder submitted to a traction-torsion test under a non-proportional strain loading path and for different material models. The simulations, based on an incremental energy minimization algorithm, highlight the evolution of both the transformation domain and the phase transformation strain rate direction. For first-order stable states, the second order stability condition is investigated in Section 6. Stability of homogeneous states are explicitly evaluated under different types of loading and closed form results are 
provided. Conclusions are drawn in Section 7

Notations. Throughout the paper, the following notations are used. $N$ denotes the dimension of the space, $1 \leq N \leq 3, N=1$ corresponds to the one-dimensional case and $N>1$ to the multi-dimensional case. In the multi-dimensional case, vectors and second order tensors are denoted by boldface letters, e.g., $\boldsymbol{u}$ for the displacement, $\boldsymbol{\sigma}$ for the stress and $\mathbf{p}$ for the phase transformation strain. Their components are denoted by the respective plain letters with lower indices like $u_{i}$ or $\sigma_{i j}, 1 \leq i, j \leq N$. The second order identity tensor is denoted 1. The summation convention is implicitly used. The inner product between vectors or between second order tensors is indicated by a dot. Accordingly, one reads $\boldsymbol{u} \cdot \boldsymbol{v}=u_{i} v_{i}, \boldsymbol{\sigma} \cdot \boldsymbol{\sigma}=\sigma_{i j} \sigma_{i j}$. The euclidean norm of a vector or a second order tensor is denoted $\|\cdot\|$ and thus $\|\mathbf{p}\|=\sqrt{\mathbf{p} \cdot \mathbf{p}}$. The trace operator for a second order tensor is denoted $\operatorname{Tr}$ and thus $\operatorname{Tr} \boldsymbol{\sigma}=\boldsymbol{\sigma} \cdot \mathbf{1}=\sigma_{i i}$. The deviatoric operator is represented by the superscript $D$ and thus $\boldsymbol{\sigma}^{D}$ denotes the deviatoric part of $\boldsymbol{\sigma}$, i.e.

$$
\boldsymbol{\sigma}^{D}=\boldsymbol{\sigma}-\frac{\operatorname{Tr} \boldsymbol{\sigma}}{N} \mathbf{1}
$$

The dependence on the time parameter $t$ is indicated by a subscript whereas the dependence on the spatial coordinate $\boldsymbol{x}$ is indicated classically by parentheses, e.g. $\boldsymbol{x} \mapsto \boldsymbol{u}_{t}(\boldsymbol{x})$ stands for the displacement field at time $t$. In general, the local state functions or material parameters are represented by serif letters, like $W$ for the total strain energy density and $\mu$ for the shear modulus. The prime denotes either the derivative with respect to phase transformation norm or the directional derivative of a functional with respect to the the specified fields, the dot stands for the time derivative, e.g.

$$
\mu^{\prime}(y)=\frac{\mathrm{d} \mu}{\mathrm{d} y}(y), \quad \mathcal{E}^{\prime}(\boldsymbol{u}, \mathbf{p}, \overline{\mathbf{p}})(\boldsymbol{\delta} \boldsymbol{u}, \boldsymbol{\delta} \mathbf{p},\|\boldsymbol{\delta} \mathbf{p}\|)=\lim _{h \rightarrow 0} \frac{\mathcal{E}(\boldsymbol{u}+h \boldsymbol{\delta} \boldsymbol{u}, \mathbf{p}+h \boldsymbol{\delta} \mathbf{p}, \overline{\mathbf{p}}+h\|\boldsymbol{\delta} \mathbf{p}\|)-\mathcal{E}(\boldsymbol{u}, \mathbf{p}, \overline{\mathbf{p}})}{h}
$$

and

$$
\dot{\mathbf{u}}_{t}(\mathbf{x})=\lim _{h \rightarrow 0} \frac{1}{h}\left(\mathbf{u}_{t+h}(\mathbf{x})-\mathbf{u}_{t}(\mathbf{x})\right)
$$

The symbol $\boldsymbol{\nabla}$ and $\nabla_{s}$ will be respectively used to indicate the spatial gradient and its symmetric part when this last makes sense. Accordingly, the total strain tensor field reads as $\varepsilon=\nabla_{s} \mathbf{u}$. The Nomenclature table summarizes the main symbols used in this article.

\section{Phenomenological approach}

\subsection{Phenomenological material response}

To justify the motivations to our energetic approach, let us start with a simple phenomenological model in a one-dimensional setting $(N=1)$ able to describe superelasticity and phase transformation. The material, initially unstressed and in a pure austenite state, undergoes a martensitic phase transformation when submitted to a strain loading path. The forward and reverse phase transformation occur at constant stresses 
$\sigma_{\mathrm{Fw}}$ and $\sigma_{\mathrm{Rv}}$, with $\sigma_{\mathrm{Fw}}>\sigma_{\mathrm{Rv}}$, and are here, for sake of simplicity, symmetric in traction and compression. The phase transformation is described through a scalar internal variable $p$, which accounts for the phase transformation strain and is assumed to be bounded by a material constant $\mathrm{p}_{1}>0$ such that $|\mathrm{p}| \leq \mathrm{p}_{1}$. A pure austenite state corresponds to $\mathrm{p}=0$ while a pure martensite state corresponds to $|\mathrm{p}|=\mathrm{p}_{1}$. For a prescribed strain $\varepsilon$ and a given value of the phase transformation strain $\mathrm{p}$, the elastic strain is given, in a small strain setting, by $\varepsilon_{e}=\varepsilon-\mathrm{p}$. The stress-strain relation at fixed $\mathrm{p}$ is simply linear elastic and reads

$$
\sigma=\mathrm{E}_{0} \varepsilon_{e}=\mathrm{E}_{0}(\varepsilon-\mathrm{p})
$$

where $\mathrm{E}_{0}$ is the Young's modulus of the material, taken as a constant in this example. The material response under a strain path is depicted schematically in Figure 1, In relation with superelasticity, we require that once the phase transformation starts $(|\mathrm{p}|>0)$, the unstressed state $(\sigma=0)$ cannot be retrieved elastically i.e. a backward phase transformation must occur. For such perfect phase transformation model, this leads to $\sigma_{\mathrm{Rv}}>0$. In a rate-independent setting, we suppose that the phase transformation laws are described by

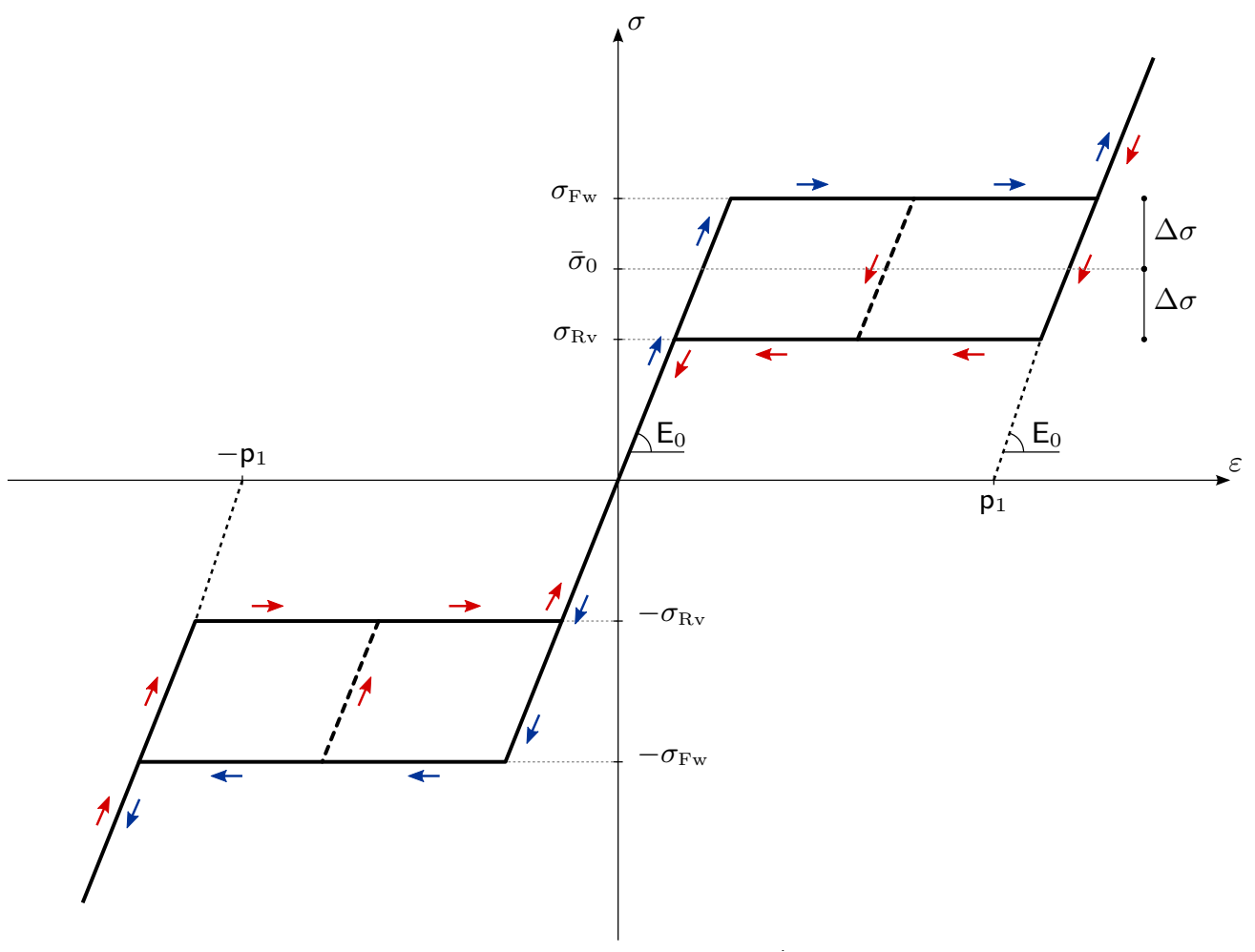

Figure 1: Schematic representation of the stress-strain response for the perfect and symmetric SMA mechanical model in the one-dimensional setting.

the following set of Karush-Kuhn-Tucker relations: 
- $\mathrm{p}=0$ :

$$
\left\{\begin{array}{l}
|\sigma| \leq \sigma_{\mathrm{Fw}}, \\
\dot{\mathrm{p}}>0 \Rightarrow \sigma=\sigma_{\mathrm{Fw}}, \\
\dot{\mathrm{p}}<0 \Rightarrow \sigma=-\sigma_{\mathrm{FW}}
\end{array}\right.
$$

- $0<\mathrm{p}<\mathrm{p}_{1}$ :

$$
\left\{\begin{array}{l}
\sigma_{\mathrm{Rv}} \leq \sigma \leq \sigma_{\mathrm{Fw}} \\
\dot{\mathbf{p}}>0 \Rightarrow \sigma=\sigma_{\mathrm{Fw}} \\
\dot{\mathbf{p}}<0 \Rightarrow \sigma=\sigma_{\mathrm{Rv}}
\end{array}\right.
$$

- $-\mathrm{p}_{1}<\mathrm{p}<0$ :

$$
\left\{\begin{array}{l}
-\sigma_{\mathrm{Fw}} \leq \sigma \leq-\sigma_{\mathrm{Rv}}, \\
\dot{\mathrm{p}}>0 \Rightarrow \sigma=-\sigma_{\mathrm{Rv}}, \\
\dot{\mathrm{p}}<0 \Rightarrow \sigma=-\sigma_{\mathrm{Fw}}
\end{array}\right.
$$

- $\mathrm{p}=\mathrm{p}_{1}$ :

$$
\left\{\begin{array}{l}
\sigma \geq \sigma_{\mathrm{Rv}}, \\
\dot{\mathbf{p}} \neq 0 \Rightarrow \dot{\mathbf{p}}<0, \sigma=\sigma_{\mathrm{Rv}}
\end{array}\right.
$$

- $\mathrm{p}=-\mathrm{p}_{1}$ :

$$
\left\{\begin{array}{l}
\sigma \leq-\sigma_{\mathrm{Rv}}, \\
\dot{\mathbf{p}} \neq 0 \Rightarrow \dot{\mathbf{p}}>0, \sigma=-\sigma_{\mathrm{Rv}}
\end{array}\right.
$$

The set of (large) inequalities in the aforementioned phase transformation laws defines the transformation domain, a closed evolving set in which the current stress must always belong to. Accordingly to the consistency conditions, the phase transformation strain $\mathrm{p}$ can evolve only if the stress is on the boundary of the transformation domain, that is, only if a stress equality holds. For rate-independent materials, the KKT relations (2)-(6) are equivalent to an associative flow-rule which defines the direction of $\dot{p}, 26$.

An important feature of such rate-independent constitutive behaviour is the possibility to reformulate the problem in a variational way. In that perspective, let us introduce the cumulated phase transformation strain $\overline{\mathrm{p}}=\overline{\mathrm{p}}_{0}+\int_{0}^{t}|\dot{\mathrm{p}}| d \tau$ from 0 to time $t$, with $\overline{\mathrm{p}}_{0}$ being the initial cumulated phase transformation strain, as well as the two material constants:

$$
\bar{\sigma}_{0}=\frac{\sigma_{\mathrm{Fw}}+\sigma_{\mathrm{Rv}}}{2}, \quad \Delta \sigma=\frac{\sigma_{\mathrm{Fw}}-\sigma_{\mathrm{Rv}}}{2}
$$

Since we assumed that $\sigma_{\mathrm{Fw}}>\sigma_{\mathrm{Rv}}$ and $\sigma_{\mathrm{Rv}}>0$, the following inequalities hold

$$
\bar{\sigma}_{0}>\Delta \sigma>0
$$


Given a triple $(\varepsilon, \mathrm{p}, \overline{\mathrm{p}})$, let us introduce the state function $W(\varepsilon, \mathrm{p}, \overline{\mathrm{p}})$ as

$$
W(\varepsilon, \mathrm{p}, \overline{\mathrm{p}})=\frac{1}{2} \mathrm{E}_{0}(\varepsilon-\mathrm{p})^{2}+\bar{\sigma}_{0}|\mathrm{p}|+\Delta \sigma \overline{\mathrm{p}}
$$

The first and the last term of the right-hand side in (9) are equal, respectively, to the elastic strain energy and the dissipation energy of a perfect-plasticity model. Instead, the second term is an additional simplified free-energy term representative of both the phase interaction energy among the phases and the effective chemical energy, [6]. The physical meanings of $\mathrm{E}_{0}, \bar{\sigma}_{0}$ and $\Delta \sigma$ are highlighted in Figure 1 . Then, we are able to reformulate (1)-(6) in the following variational form:

Proposition 2.1. The evolution problem formulated in terms of the stress-strain relation and the phase transformation laws (1)-(6) is equivalent to the variational problem

$$
\begin{aligned}
\text { Stress-strain relation: } & \sigma=\frac{\partial W}{\partial \varepsilon}(\varepsilon, \mathrm{p}, \overline{\mathrm{p}}) \\
\text { Material stability: } & \forall \mathrm{p}^{*} \quad \text { with } \quad\left|\mathrm{p}^{*}\right| \leq \mathrm{p}_{1}: \quad W(\varepsilon, \mathrm{p}, \overline{\mathrm{p}}) \leq W\left(\varepsilon, \mathrm{p}^{*}, \overline{\mathrm{p}}+\left|\mathrm{p}^{*}-\mathrm{p}\right|\right) \\
\text { Energy balance: } & W(\varepsilon, \mathrm{p}, \overline{\mathrm{p}})=W\left(\varepsilon_{0}, \mathrm{p}_{0}, \overline{\mathrm{p}}_{0}\right)+\int_{0}^{t} \sigma \dot{\varepsilon} \mathrm{d} \tau
\end{aligned}
$$

Proof. See Appendix 1.

The evolution laws of a material point are hence characterized by two physical principles:

- a material stability criterion which selects the minima of $W$ with respect to $(\mathrm{p}, \overline{\mathrm{p}})$ for a given $\varepsilon$.

- an energy balance condition which defines $W$ as the sum of the total strain work $\int_{0}^{t} \sigma \dot{\varepsilon} d \tau$ and the total strain energy density at initial time $W\left(\varepsilon_{0}, \mathrm{p}_{0}, \overline{\mathrm{p}}_{0}\right)$. Thus, $W$ correspond to the total strain energy density of the system at time $t$.

In particular, such variational formulation allows to write in a more condensed way the several cases in (2)-(6) that has to be distinguished depending on the value of $\mathrm{p}$.

Remark 1. Such kind of total strain energy density has strong analogies with those investigated by the coauthors [32, 3] about damage and coupled damage-plasticity models. But above all, the strongest analogies are with the classical multi-dimensional Von-Mises model of perfect incompressible plasticity, which is built on the following total strain energy density, [12],

$$
W(\varepsilon, \mathbf{p}, \overline{\mathbf{p}})=\mu\left(\varepsilon^{D}-\mathbf{p}\right) \cdot\left(\varepsilon^{D}-\mathbf{p}\right)+\frac{1}{2} K(\operatorname{Tr}(\varepsilon))^{2}+\sigma_{e} \overline{\mathbf{p}}
$$

where $\sigma_{e}>0, \mathbf{p}$ and $\overline{\mathbf{p}}$ are respectively the Von Mises yield stress, the plastic strain and the cumulated plastic strain. In the perfect Von-Mises plasticity model the dissipated energy is due only to plasticity and reads $\sigma_{e} \overline{\mathbf{p}}$. 
The above example is a simplified model which aims to introduce and motivate, in addition to what has been already stated in the introduction, our choice to work with the total strain energy of the system and deal with energetic variational concepts. The perfect phase transformation behaviour has obvious limitations in terms of possible choices of stress-strain responses. Specifically, one-dimensional experiments on polycrystalline SMA exhibits a more complex macroscopic behaviour with a loss of stiffness along with a stress-hardening and/or stress-softening behaviour, [17. To broaden the class of phenomena that can be captured, a more general model than (9) should be considered. In this energetic framework, a way to extend the model is to modify directly the strain energy density, on which the stability criterion and energy balance conditions are based. A possible choice for an enriched strain energy density $W$, able to describe both stiffness variations and hardening/softening behaviours, is the following:

$$
W(\varepsilon, \mathrm{p}, \overline{\mathrm{p}})=\frac{1}{2} \mathrm{E}(|\mathrm{p}|)(\varepsilon-\mathrm{p})^{2}+\bar{\sigma}(|\mathrm{p}|)+\Delta \sigma \overline{\mathrm{p}}
$$

where $\mathrm{E}:\left[0, \mathrm{p}_{1}\right] \mapsto \mathbb{R}^{+}$and $\bar{\sigma}:\left[0, \mathrm{p}_{1}\right] \mapsto \mathbb{R}^{+}$are now material functions. The loss of stiffness during martensitic transformation is then understood as

$$
\mathrm{E}^{\prime}(|\mathrm{p}|)<0 \quad \forall \mathrm{p} \in\left[0, \mathrm{p}_{1}\right]
$$

Moreover, we recall that the zero-stress state must be necessarily retrieved through a dissipative process in superelastic SMA once the martensitic transformation has been triggered. In other words, $\sigma=0$ must not belong to the transformation domain when $\mathrm{p}>0$. Based on a direct calculation of the transformation domain based on 11 , such condition leads to

$$
\bar{\sigma}^{\prime}(|\mathrm{p}|)>\Delta \sigma \quad \forall \mathrm{p} \in\left[0, \mathrm{p}_{1}\right]
$$

which is a generalization of [8] for a non-perfect behaviour.

The purpose of next sections is to derive the evolution laws in a multi-dimensional setting, starting from a strain energy of the kind of 14, with a systematic use of a stability criterion and an energy balance condition.

Remark 2. To identify the two material functions $y \mapsto \mathrm{E}(y)$ and $y \mapsto \bar{\sigma}(y)$ as well as the material constant $\Delta \sigma$, one needs at least two experimental curves. From a uniaxial tensile test on a material point submitted to a loading-unloading, two stress-strain curves can be extracted, namely the forward stress-strain response $\sigma=\mathcal{F}(\varepsilon)$ and the backward stress-strain response $\sigma=\mathcal{B}(\varepsilon)$. Such curves allow to identify the material functions. Indeed, a direct calculation (see [30] for the detailed calculation) of the forward and backward material response of the model (14) shows that the strain and the stress during the forward transformation are given by in terms of the transformation strain $\mathrm{p}$ by

$$
\varepsilon_{F}(p)=p-\frac{E(p)}{E^{\prime}(p)}\left(\sqrt{1-2 \frac{E^{\prime}(p)\left(\bar{\sigma}^{\prime}(p)+\Delta \sigma\right)}{(E(p))^{2}}}-1\right), \quad \sigma_{F}(p)=E(p)\left(\varepsilon_{F}(p)-p\right)
$$


while the strain and the stress during the backward transformation are given by

$$
\varepsilon_{B}(p)=p-\frac{E(p)}{E^{\prime}(p)}\left(\sqrt{1-2 \frac{E^{\prime}(p)\left(\bar{\sigma}^{\prime}(p)-\Delta \sigma\right)}{(E(p))^{2}}}-1\right), \quad \sigma_{B}(p)=E(p)\left(\varepsilon_{B}(p)-p\right) .
$$

Therefore we deduce that the $\mathcal{F}=\sigma_{F} \circ \varepsilon_{F}^{-1}$ and $\mathcal{B}=\sigma_{B} \circ \varepsilon_{B}^{-1}$. As a result, one have to choose optimally $y \mapsto \mathrm{E}(y)$ and $y \mapsto \bar{\sigma}(y)$ to ensure such relations. In addition, $\Delta \sigma$ can be identified by computing to the total dissipated energy in a strain cycle which corresponds to the hysteresis loop area in the stress-strain diagram i.e. $\oint \sigma \mathrm{d} \varepsilon=2 \Delta \sigma \mathrm{p}_{1}$. This identification procedure is possible provided that one can extract such material response from a structural test. This is straightforward if the response of the body is homogeneous. However, for SMA with a stress-softening behavior, experiments show that the homogeneous response under uniaxial loading are unobservable and require special apparatus to stabilize it [17]. This is confirmed theoretically by a stability analysis in a one-dimensional setting [30, 1]. In Section 6, we will show through a stability analysis in the multi-dimensional setting that a way to overcome such instabilities issues in uniaxial tests is to choose instead non-proportional loading path for which the homogeneous response is shown to be stable.

\section{Formulation of the model and evolution problem}

\subsection{Hypotheses and density of strain energy}

Generalizing the previous one-dimensional example, we suppose that a material point, in a $N$-dimensional setting $(N=2$ or 3$)$, is described at time $t$ by the following three state variables:

- a total strain variable $\varepsilon \in \mathbb{M}_{s}^{N}$;

- a phase transformation strain variable $\mathbf{p} \in \mathbb{M}_{s}^{N}$ bounded by a maximum value $\mathrm{p}_{1}>0$ i.e. $\|\mathbf{p}\| \leq \mathrm{p}_{1}$. Since the hydrostatic pressure has little influence on the phase transformation in SMA [16], we also assume that $\mathbf{p}$ is deviatoric i.e. $\operatorname{Tr}(\mathbf{p})=0$;

- a hardening variable $\overline{\mathrm{p}} \in \mathbb{R}^{+}$defined as the cumulated phase transformation strain: $\overline{\mathrm{p}}=\overline{\mathrm{p}}_{0}+\int_{0}^{t}\|\dot{\mathbf{p}}\| d \tau$ for a process occurring from 0 to time $t$ with $\overline{\mathrm{p}}_{0}$ being the initial cumulated phase transformation strain.

Motivated by the variational setting of the one-dimensional model, we directly postulate the total strain energy of the three-dimensional model generalizing 14 as a function of the state $(\varepsilon, \mathbf{p}, \overline{\mathbf{p}})$ :

$$
W(\varepsilon, \mathbf{p}, \overline{\mathbf{p}})=\mu(\|\mathbf{p}\|)\left(\varepsilon^{D}-\mathbf{p}\right) \cdot\left(\varepsilon^{D}-\mathbf{p}\right)+\frac{1}{2} K(\operatorname{Tr}(\varepsilon))^{2}+\bar{\sigma}(\|\mathbf{p}\|)+\Delta \sigma \overline{\mathbf{p}}
$$

Such model depends on four scalar material parameters, namely:

- a shear modulus $\mu:\left[0, \mathrm{p}_{1}\right] \mapsto \mathbb{R}^{+}$taken as a material function with $\mu^{\prime}<0$; 
- a bulk modulus $K$ taken as a material constant because of the phase transformation incompressibility, [16];

- a material constant $\Delta \sigma>0$;

- a material function $\bar{\sigma}:\left[0, \mathrm{p}_{1}\right] \mapsto \mathbb{R}$ with $\bar{\sigma}^{\prime}>\Delta \sigma$;

\subsection{Evolution problem}

We consider the quasi-static time evolution problem of an open bounded domain $\Omega \in \mathbb{R}^{n}$ in absence of body forces and obeying to a phase transformation model characterized by the total strain energy density (19). The time evolution parameter is denoted by $t$ with $t=0$ being the initial time instant. Such domain is submitted to time-dependent prescribed displacement $\boldsymbol{U}_{\boldsymbol{d}}$ on a part $\partial_{D} \Omega$ and prescribed tractions $\boldsymbol{T}_{\boldsymbol{d}}$ on the remaining part $\partial_{T} \Omega$. Let us first introduce the admissible spaces of displacement $\mathcal{C}$ and displacement rate $\mathcal{C}_{0}$ which respectively read as

$$
\mathcal{C}=\left\{\boldsymbol{u} \in H^{1}\left(\Omega, \mathbb{R}^{n}\right): \boldsymbol{u}=\boldsymbol{U}_{\boldsymbol{d}} \text { on } \partial_{D} \Omega\right\}, \quad \mathcal{C}_{0}=\left\{\boldsymbol{u} \in H^{1}\left(\Omega, \mathbb{R}^{n}\right): \boldsymbol{u}=0 \text { on } \partial_{D} \Omega\right\}
$$

with $H^{1}$ being the Sobolev space of functions which are square integrable and the weak derivative of which is also square integrable. The admissible spaces of phase transformation strain $\mathcal{Z}$ and phase transformation strain rate $\mathcal{Z}_{0}(\mathbf{p})$ are respectively defined as

$$
\begin{aligned}
& \mathcal{Z}=\left\{\mathbf{p} \in L^{\infty}\left(\Omega, \mathbb{M}_{s}^{N}\right): \operatorname{Tr}(\mathbf{p})=0 ; \quad\|\mathbf{p}\| \leq \mathrm{p}_{1}\right\} \\
& \mathcal{Z}_{0}(\mathbf{p})=\left\{\boldsymbol{\delta} \mathbf{p} \in L^{\infty}\left(\Omega, \mathbb{M}_{s}^{N}\right): \operatorname{Tr}(\boldsymbol{\delta} \mathbf{p})=0 ; \quad \boldsymbol{\delta} \mathbf{p} \cdot \mathbf{p} \leq 0 \text { if }\|\mathbf{p}\|=\mathrm{p}_{1}\right\}
\end{aligned}
$$

while the admissible space of cumulated phase transformation strain reads

$$
\overline{\mathcal{Z}}=L^{1}\left(\Omega, \mathbb{R}^{+}\right)
$$

with $L^{1}$ and $L^{\infty}$ being, respectively, the space of integrable functions and the space of essentially bounded integrable functions.

At initial time $t=0$, the domain is supposed to be in an admissible given state $\left(\boldsymbol{u}_{0}, \mathbf{p}_{0}, \overline{\mathrm{p}}_{0}\right) \in \mathcal{C} \times \mathcal{Z} \times \overline{\mathcal{Z}}$. For a given set of admissible variables $(\boldsymbol{u}, \mathbf{p}, \overline{\mathrm{p}}) \in \mathcal{C} \times \mathcal{Z} \times \overline{\mathcal{Z}}$ at time $t$, the total energy of the system is given by

$$
\mathcal{E}(\boldsymbol{u}, \mathbf{p}, \overline{\mathrm{p}})=\int_{\Omega} W(\varepsilon(\boldsymbol{u})(x), \mathbf{p}(x), \overline{\mathrm{p}}(x)) \mathrm{d} x-\int_{\partial_{T} \Omega} \boldsymbol{T}_{\boldsymbol{d}}(x) \cdot \boldsymbol{u}(x) \mathrm{d} x
$$

where $\boldsymbol{\varepsilon}(\boldsymbol{u})=\nabla_{s} \boldsymbol{u}$. The quasi-static evolution problem of the domain $\Omega$ is formulated energetically at the global level. Following the material variational formulation (11)-(12), the structural evolution problem is

\footnotetext{
${ }^{1} \boldsymbol{\delta} \mathbf{p}$ will be an admissible test direction from $\mathbf{p}$ when $\|\mathbf{p}\|=\mathrm{p}_{1}$ if and only if $\lim _{h \rightarrow 0^{+}} \frac{\|\mathbf{p}+h \boldsymbol{\delta} \mathbf{p}\|-\|\mathbf{p}\|}{h} \leq 0$
} 
based on two physical principles, namely a stability criterion and an energy balance condition. Here, the stability criterion consists in selecting the directional local minima of the total energy at each time and is formally defined as

Definition 3.1 (Stability criterion). A state $(\boldsymbol{u}, \mathbf{p}, \overline{\mathrm{p}}) \in \mathcal{C} \times \mathcal{Z} \times \overline{\mathcal{Z}}$ will be locally directionally stable if for a given set of test directions $(\boldsymbol{\delta} \boldsymbol{u}, \boldsymbol{\delta} \mathbf{p}) \in \mathcal{C}_{0} \times \mathcal{Z}_{0}$, there exists $r>0$ such that for any $h \leq r$ we have

$$
\mathcal{E}(\boldsymbol{u}, \mathbf{p}, \overline{\mathrm{p}}) \leq \mathcal{E}(\boldsymbol{u}+h \boldsymbol{\delta} \boldsymbol{u}, \mathbf{p}+h \boldsymbol{\delta} \mathbf{p}, \overline{\mathrm{p}}+h\|\boldsymbol{\delta} \mathbf{p}\|)
$$

Note that compared to the material stability criterion (11), the structural stability criterion (24) is slightly different. For instance, it is more selective in the sense that it looks for local minima with respect to the triplet $(\boldsymbol{u}, \mathbf{p}, \overline{\mathbf{p}})$ and not the pair $(\mathbf{p}, \overline{\mathbf{p}})$. On the other hand it is less restrictive in the sense that we choose to select directional local minima instead of global minima. Alternative criteria based on global minima or local minima with a different topology can be considered, but are here not investigated.

The energy balance condition enforces the time evolution to be consistent with the applied loading in terms of energy exchanges, [26, 15, 31, 2]. In a quasi-static setting, it reads as

Definition 3.2 (Energy balance condition). The total energy of the system satisfies the energy balance condition if the following equality holds at any instant $t$

$$
\mathcal{E}(\boldsymbol{u}, \mathbf{p}, \overline{\mathrm{p}})=\mathcal{E}\left(\boldsymbol{u}_{0}, \mathbf{p}_{0}, \overline{\mathrm{p}}_{0}\right)+\int_{0}^{t}\left(\int_{\partial_{D} \Omega} \boldsymbol{T} \cdot \dot{\boldsymbol{U}}_{\boldsymbol{d}} \mathrm{d} x-\int_{\partial_{T} \Omega} \dot{\boldsymbol{T}}_{\boldsymbol{d}} \cdot \boldsymbol{u} \mathrm{d} x\right) \mathrm{d} \tau
$$

where $\boldsymbol{T}$ is the force vector on the boundary $\partial_{D} \Omega$.

Similarly to the stability criterion, the energy balance condition is a global version of the material energy balance condition introduced in 12 .

\section{Evolution laws: Phase transformation criteria and consistency conditions}

In this section, we derive the local evolution laws based on the stability criterion and the energy balance condition. For a given state $(\boldsymbol{u}, \mathbf{p}, \overline{\mathrm{p}}) \in \mathcal{C} \times \mathcal{Z} \times \overline{\mathcal{Z}}$ and test direction $(\boldsymbol{\delta} \boldsymbol{u}, \boldsymbol{\delta} \mathbf{p}) \in \mathcal{C}_{0} \times \mathcal{Z}_{0}$, the series expansion of the total energy $\mathcal{E}(\boldsymbol{u}+h \boldsymbol{\delta} \boldsymbol{u}, \mathbf{p}+h \boldsymbol{\delta} \mathbf{p}, \overline{\mathrm{p}}+h\|\boldsymbol{\delta} \mathbf{p}\|)$, for $h>0$ sufficiently small, gives

$$
\begin{aligned}
\mathcal{E}(\boldsymbol{u}+h \boldsymbol{\delta} \boldsymbol{u}, \mathbf{p}+h \boldsymbol{\delta} \mathbf{p}, \overline{\mathrm{p}}+h\|\boldsymbol{\delta} \mathbf{p}\|)= & \mathcal{E}(\boldsymbol{u}, \mathbf{p}, \overline{\mathrm{p}}) \\
& +h \mathcal{E}^{\prime}(\boldsymbol{u}, \mathbf{p}, \overline{\mathrm{p}})(\boldsymbol{\delta} \boldsymbol{u}, \boldsymbol{\delta} \mathbf{p},\|\boldsymbol{\delta} \mathbf{p}\|) \\
& +\frac{h^{2}}{2} \mathcal{E}^{\prime \prime}(\boldsymbol{u}, \mathbf{p}, \overline{\mathrm{p}})(\boldsymbol{\delta} \boldsymbol{u}, \boldsymbol{\delta} \mathbf{p},\|\boldsymbol{\delta} \mathbf{p}\|)+o\left(h^{2}\right)
\end{aligned}
$$

Based on such expansion, let us introduce 
Definition 4.1 (First- and Second-order stability condition). A state $(\boldsymbol{u}, \mathbf{p}, \overline{\mathbf{p}}) \in \mathcal{C} \times \mathcal{Z} \times \overline{\mathcal{Z}}$ is said to satisfy the First-order condition of stability if the first-order derivative of the total energy is positive in any test direction:

$$
\forall(\boldsymbol{\delta} \boldsymbol{u}, \boldsymbol{\delta} \mathbf{p}) \in \mathcal{C}_{0} \times \mathcal{Z}_{0}, \quad \mathcal{E}^{\prime}(\boldsymbol{u}, \mathbf{p}, \overline{\mathbf{p}})(\boldsymbol{\delta} \boldsymbol{u}, \boldsymbol{\delta} \mathbf{p},\|\boldsymbol{\delta} \mathbf{p}\|) \geq 0
$$

A state will satisfy the Second-order condition of stability if for any test direction such that the first-order derivative is zero, the second-order derivative of the total energy is positive:

$$
\forall(\boldsymbol{\delta} \boldsymbol{u}, \boldsymbol{\delta} \mathbf{p}) \in \mathcal{C}_{0} \times \mathcal{Z}_{0}, \quad \mathcal{E}^{\prime}(\boldsymbol{u}, \mathbf{p}, \overline{\mathrm{p}})(\boldsymbol{\delta} \boldsymbol{u}, \boldsymbol{\delta} \mathbf{p},\|\boldsymbol{\delta} \mathbf{p}\|)=0 \quad \Rightarrow \quad \mathcal{E}^{\prime \prime}(\boldsymbol{u}, \mathbf{p}, \overline{\mathrm{p}})(\boldsymbol{\delta} \boldsymbol{u}, \boldsymbol{\delta} \mathbf{p},\|\boldsymbol{\delta} \mathbf{p}\|) \geq 0
$$

Upon the introduction of such definitions, we readily obtain

Proposition 4.2 (Necessary and sufficient conditions of directional stability). A state $(\boldsymbol{u}, \mathbf{p}, \overline{\mathrm{p}}) \in \mathcal{C} \times \mathcal{Z} \times \overline{\mathcal{Z}}$ will be directionally stable only if it fulfills the first- and second-order conditions of stability. Reciprocally, a state will be directionally stable if for any test direction $(\boldsymbol{\delta} \boldsymbol{u}, \boldsymbol{\delta} \mathbf{p}) \in \mathcal{C}_{0} \times \mathcal{Z}_{0}$, either

$$
\mathcal{E}^{\prime}(\boldsymbol{u}, \mathbf{p}, \overline{\mathbf{p}})(\boldsymbol{\delta} \boldsymbol{u}, \boldsymbol{\delta} \mathbf{p},\|\boldsymbol{\delta} \mathbf{p}\|)>0
$$

or

$$
\mathcal{E}^{\prime \prime}(\boldsymbol{u}, \mathbf{p}, \overline{\mathrm{p}})(\boldsymbol{\delta} \boldsymbol{u}, \boldsymbol{\delta} \mathbf{p},\|\boldsymbol{\delta} \mathbf{p}\|)>0 \quad \text { if } \quad \mathcal{E}^{\prime}(\boldsymbol{u}, \mathbf{p}, \overline{\mathrm{p}})(\boldsymbol{\delta} \boldsymbol{u}, \boldsymbol{\delta} \mathbf{p},\|\boldsymbol{\delta} \mathbf{p}\|)=0
$$

hold.

The condition $\mathcal{E}^{\prime}=0$ is also generally known as a stationary condition.

\subsection{Derivations of phase transformation criteria and consistency conditions}

We derive in this section phase transformation criteria from the first-order stability condition.

Proposition 4.3 (Phase transformation criteria). A state $(\boldsymbol{u}, \mathbf{p}, \overline{\mathrm{p}}) \in \mathcal{C} \times \mathcal{Z} \times \overline{\mathcal{Z}}$ will satisfy the first-order condition of stability if and only if it satisfies the stress-strain constitutive relation

$$
\boldsymbol{\sigma}=2 \mu(\|\mathbf{p}\|)\left(\varepsilon^{D}-\mathbf{p}\right)+K \operatorname{Tr}(\varepsilon) \mathbf{1}
$$

the mechanical equilibrium

$$
\boldsymbol{\nabla} \cdot \boldsymbol{\sigma}=\mathbf{0}, \quad \text { in } \Omega \quad \text { and } \quad \boldsymbol{\sigma} \boldsymbol{n}=\boldsymbol{T}_{\boldsymbol{d}} \quad \text { on } \partial_{T} \Omega
$$

as well as the following phase transformation criteria:

- on the subset $\Omega_{0}$ of $\Omega$ where $\|\mathbf{p}\|=0$ :

$$
\left\|\boldsymbol{\sigma}^{D}\right\| \leq \Delta \sigma+R\left(0,\left\|\boldsymbol{\sigma}^{D}\right\|\right)
$$


- on the subset $\Omega_{\mathrm{p}}$ of $\Omega$ where $0<\|\mathbf{p}\|<\mathrm{p}_{1}$ :

$$
\left\|\boldsymbol{\sigma}^{D}-R\left(\|\mathbf{p}\|,\left\|\boldsymbol{\sigma}^{D}\right\|\right) \boldsymbol{n}_{\mathbf{p}}\right\| \leq \Delta \sigma
$$

- on the subset $\Omega_{1}^{-}$of $\Omega$ where $\|\mathbf{p}\|=\mathrm{p}_{1}$ and $\mathbf{p} \cdot\left(\boldsymbol{\sigma}^{D}-R\left(\mathbf{p}_{1},\left\|\boldsymbol{\sigma}^{D}\right\|\right) \boldsymbol{n}_{\mathbf{p}}\right) \leq 0$ :

$$
\left\|\boldsymbol{\sigma}^{D}-R\left(\mathbf{p}_{1},\left\|\boldsymbol{\sigma}^{D}\right\|\right) \boldsymbol{n}_{\mathbf{p}}\right\| \leq \Delta \sigma
$$

- on the subset $\Omega_{1}^{+}$of $\Omega$ where $\|\mathbf{p}\|=\mathrm{p}_{1}$ and $\mathbf{p} \cdot\left(\boldsymbol{\sigma}^{D}-R\left(\mathrm{p}_{1},\left\|\boldsymbol{\sigma}^{D}\right\|\right) \boldsymbol{n}_{\mathbf{p}}\right)>0$ :

$$
\left\|\operatorname{proj}_{\perp \mathbf{p}}\left(\boldsymbol{\sigma}^{D}-R\left(\mathrm{p}_{1},\left\|\boldsymbol{\sigma}^{D}\right\|\right) \boldsymbol{n}_{\mathbf{p}}\right)\right\| \leq \Delta \sigma
$$

with $\boldsymbol{n}_{\mathbf{p}}=\mathbf{p} /\|\mathbf{p}\|$, the scalar function $R\left(y_{1}, y_{2}\right)=\bar{\sigma}^{\prime}\left(y_{1}\right)-\left(\left(y_{2}\right)^{2} / 4\right)(1 / \mu)^{\prime}\left(y_{1}\right)$ and $\operatorname{proj}_{\perp \mathbf{p}}(\cdot)$ being the orthogonal projection to $\mathbf{p}$.

Proof. We first assume that the first-order stability condition holds and deduce the phase transformation criteria. A direct calculation of the first-order derivative of the total energy $(19)$ and 23$]$ in the test direction $(\boldsymbol{\delta} \boldsymbol{u}, \boldsymbol{\delta} \mathbf{p}) \in \mathcal{C}_{0} \times \mathcal{Z}_{0}$ gives

$$
\begin{aligned}
\mathcal{E}^{\prime}(\boldsymbol{u}, \mathbf{p}, \overline{\mathrm{p}})(\boldsymbol{\delta} \boldsymbol{u}, \boldsymbol{\delta} \mathbf{p},\|\boldsymbol{\delta} \mathbf{p}\|)= & \mathcal{E}^{\prime}(\boldsymbol{u}, \mathbf{p}, \overline{\mathrm{p}})(\boldsymbol{\delta} \boldsymbol{u}, \mathbf{0}, \mathbf{0})+\mathcal{E}^{\prime}(\boldsymbol{u}, \mathbf{p}, \overline{\mathrm{p}})(\mathbf{0}, \boldsymbol{\delta} \mathbf{p},\|\boldsymbol{\delta} \mathbf{p}\|)= \\
& \int_{\Omega} \boldsymbol{\sigma} \cdot \boldsymbol{\varepsilon}(\boldsymbol{\delta} \boldsymbol{u}) d x-\int_{\partial_{T} \Omega} \boldsymbol{T}_{\boldsymbol{d}} \cdot \boldsymbol{\delta} \boldsymbol{u} \mathrm{d} x \\
& +\int_{\Omega_{0}}\left(-\boldsymbol{\sigma}^{D} \cdot \boldsymbol{\delta} \mathbf{p}+\left(\Delta \sigma+R\left(0,\left\|\boldsymbol{\sigma}^{D}\right\|\right)\right)\|\boldsymbol{\delta} \mathbf{p}\|\right) \mathrm{d} x \\
& +\int_{\Omega_{\mathrm{p}}}\left(-\boldsymbol{\sigma}^{D} \cdot \boldsymbol{\delta} \mathbf{p}+R\left(\|\mathbf{p}\|,\left\|\boldsymbol{\sigma}^{D}\right\|\right) \boldsymbol{n}_{\mathbf{p}} \cdot \boldsymbol{\delta} \mathbf{p}+\Delta \sigma\|\boldsymbol{\delta} \mathbf{p}\|\right) \mathrm{d} x \\
& +\int_{\Omega_{1}^{+} \cup \Omega_{1}^{-}}\left(-\boldsymbol{\sigma}^{D} \cdot \boldsymbol{\delta} \mathbf{p}+R\left(\mathrm{p}_{1},\left\|\boldsymbol{\sigma}^{D}\right\|\right) \boldsymbol{n}_{\mathbf{p}} \cdot \boldsymbol{\delta} \mathbf{p}+\Delta \sigma\|\boldsymbol{\delta} \mathbf{p}\|\right) \mathrm{d} x
\end{aligned}
$$

with $\boldsymbol{\sigma}$ given by (31) and $\boldsymbol{\sigma} \cdot \boldsymbol{\delta} \mathbf{p}=\boldsymbol{\sigma}^{D} \cdot \boldsymbol{\delta} \mathbf{p}$ since $\boldsymbol{\delta} \mathbf{p}$ is deviatoric.

Mechanical Equilibrium. By setting $\boldsymbol{\delta} \mathbf{p}=\mathbf{0}$, the first-order stability condition reduces to

$$
\forall \boldsymbol{\delta} \boldsymbol{u} \in \mathcal{C}_{0}, \quad \mathcal{E}^{\prime}(\boldsymbol{u}, \mathbf{p}, \overline{\mathrm{p}})(\boldsymbol{\delta} \boldsymbol{u}, \mathbf{0}, \mathbf{0})=\int_{\Omega} \boldsymbol{\sigma} \cdot \boldsymbol{\varepsilon}(\boldsymbol{\delta} \boldsymbol{u}) \mathrm{d} x-\int_{\partial_{T} \Omega} \boldsymbol{T}_{\boldsymbol{d}} \cdot \boldsymbol{\delta} \boldsymbol{u} \mathrm{d} x \geq 0
$$

Having the choice of the sign of the test direction $\boldsymbol{\delta} \boldsymbol{u}$, inequality 38 is in fact an equality valid for any $\boldsymbol{\delta} \boldsymbol{u} \in \mathcal{C}_{0}$. It is precisely the weak formulation of the mechanical equilibrium 32 . This leads to

$$
\forall \boldsymbol{\delta} \boldsymbol{u} \in \mathcal{C}_{0}, \quad \mathcal{E}^{\prime}(\boldsymbol{u}, \mathbf{p}, \bar{p})(\boldsymbol{\delta} \boldsymbol{u}, \boldsymbol{\delta} \mathbf{p},\|\boldsymbol{\delta} \mathbf{p}\|)=\mathcal{E}^{\prime}(\boldsymbol{u}, \mathbf{p}, \overline{\mathbf{p}})(\mathbf{0}, \boldsymbol{\delta} \mathbf{p},\|\boldsymbol{\delta} \mathbf{p}\|)
$$


Phase transformation criterion in $\Omega_{0}$. We now consider test directions $\boldsymbol{\delta} \mathbf{p}$ which are zero on $\Omega_{\mathrm{p}} \cup \Omega_{1}^{+} \cup \Omega_{1}^{-}$. The first-order stability condition reads for such $\boldsymbol{\delta} \mathbf{p}$

$$
\mathcal{E}^{\prime}(\boldsymbol{u}, \mathbf{p}, \overline{\mathbf{p}})(\mathbf{0}, \boldsymbol{\delta} \mathbf{p},\|\boldsymbol{\delta} \mathbf{p}\|)=\int_{\Omega_{0}}\left(-\boldsymbol{\sigma}^{D} \cdot \boldsymbol{\delta} \mathbf{p}+\left(\Delta \sigma+R\left(0,\left\|\boldsymbol{\sigma}^{D}\right\|\right)\right)\|\boldsymbol{\delta} \mathbf{p}\|\right) \mathrm{d} x \geq 0
$$

Such variational inequality enforces $(33)$ in $\Omega_{0}$. Indeed, by contradiction, let us assume that it exists a subset $\Omega_{0}^{*}$ of $\Omega_{0}$ of non-zero measure where $\left\|\boldsymbol{\sigma}^{D}\right\|>\Delta \sigma+R\left(0,\left\|\boldsymbol{\sigma}^{D}\right\|\right)$ (for sake of clarity, we omit the spatial dependence in the variable $x$ of $\boldsymbol{\sigma}^{D}(x)$ and $\mathbf{p}(x)$ ). We necessarily have $\left\|\boldsymbol{\sigma}^{D}\right\|>0$ since $\bar{\sigma}^{\prime}(0) \geq-\Delta \sigma$ by hypothesis. Then by taking the particular test direction $\delta \mathbf{p}^{*}$ as equals to $\boldsymbol{\sigma}^{D} /\left\|\boldsymbol{\sigma}^{D}\right\|$ (hence being deviatoric) in $\Omega_{0}^{*}$ and zero elsewhere, we would have

$$
\mathcal{E}^{\prime}(\boldsymbol{u}, \mathbf{p}, \overline{\mathbf{p}})\left(\mathbf{0}, \boldsymbol{\delta} \mathbf{p}^{*},\left\|\boldsymbol{\delta} \mathbf{p}^{*}\right\|\right)=\int_{\Omega_{0}^{*}}\left(-\left\|\boldsymbol{\sigma}^{D}\right\|+\left(\Delta \sigma+R\left(0,\left\|\boldsymbol{\sigma}^{D}\right\|\right)\right)\right) \mathrm{d} x<0
$$

which is in contradiction with 40.

Phase transformation criterion in $\Omega_{\mathbf{p}}$. We now consider test directions $\boldsymbol{\delta} \mathbf{p}$ which are zero on $\Omega_{0} \cup \Omega_{1}^{+} \cup \Omega_{1}^{-}$. The first-order stability condition reads for such $\delta \mathbf{p}$

$$
\mathcal{E}^{\prime}(\boldsymbol{u}, \mathbf{p}, \overline{\mathbf{p}})(\mathbf{0}, \boldsymbol{\delta} \mathbf{p},\|\boldsymbol{\delta} \mathbf{p}\|)=\int_{\Omega_{\mathrm{p}}}\left(-\boldsymbol{\sigma}^{D} \cdot \boldsymbol{\delta} \mathbf{p}+R\left(\|\mathbf{p}\|,\left\|\boldsymbol{\sigma}^{D}\right\|\right) \boldsymbol{n}_{\mathbf{p}} \cdot \boldsymbol{\delta} \mathbf{p}+\Delta \sigma\|\boldsymbol{\delta} \mathbf{p}\|\right) \mathrm{d} x \geq 0
$$

As previously, we show that this variational inequality leads to 34 in $\Omega_{\mathrm{p}}$. Indeed, let us proceed again by contradiction by assuming the existence of a non-empty subset $\Omega_{\mathrm{p}}^{*}$ of $\Omega_{\mathrm{p}}$ where $\left\|\boldsymbol{\sigma}^{D}-R\left(\|\mathbf{p}\|,\left\|\boldsymbol{\sigma}^{D}\right\|\right) \boldsymbol{n}_{\mathbf{p}}\right\|>$ $\Delta \sigma$. Then, by taking the particular test direction $\delta \mathbf{p}^{*}$ as equals to $\boldsymbol{\sigma}^{D}-R\left(\|\mathbf{p}\|,\left\|\boldsymbol{\sigma}^{D}\right\|\right) \boldsymbol{n}_{\mathbf{p}}$ divided by its norm (which is non-zero as its norm is greater that $\Delta \sigma>0$ by hypothesis) on $\Omega_{\mathrm{p}}^{*}$ and zero elsewhere, we would also have

$$
\mathcal{E}^{\prime}(\boldsymbol{u}, \mathbf{p}, \overline{\mathrm{p}})\left(\mathbf{0}, \boldsymbol{\delta} \mathbf{p}^{*},\left\|\boldsymbol{\delta} \mathbf{p}^{*}\right\|\right)=\int_{\Omega_{\mathrm{p}}^{*}}\left(-\left\|\boldsymbol{\sigma}^{D}-R\left(\|\mathbf{p}\|,\left\|\boldsymbol{\sigma}^{D}\right\|\right) \boldsymbol{n}_{\mathbf{p}}\right\|+\Delta \sigma\right) \mathrm{d} x<0
$$

which is in contradiction with 42 .

Phase transformation criterion in $\Omega_{1}^{+} \cup \Omega_{1}^{-}$. Finally, we consider test directions $\delta \mathbf{p}$ which are zero on $\Omega_{0} \cup \Omega_{\mathrm{p}}$. In $\Omega_{1}^{+} \cup \Omega_{1}^{-}$, due to the constraint $\|\mathbf{p}\| \leq \mathrm{p}_{1}$, we must have $\boldsymbol{\delta} \mathbf{p} \cdot \mathbf{p} \leq 0$ whenever $\|\mathbf{p}\|=\mathrm{p}_{1}$. The first-order stability condition reads for such $\delta \mathbf{p}$

$$
\mathcal{E}^{\prime}(\boldsymbol{u}, \mathbf{p}, \overline{\mathrm{p}})(\mathbf{0}, \boldsymbol{\delta} \mathbf{p},\|\boldsymbol{\delta} \mathbf{p}\|)=\int_{\Omega_{1}^{+} \cup \Omega_{1}^{-}}\left(-\boldsymbol{\sigma}^{D} \cdot \boldsymbol{\delta} \mathbf{p}+R\left(\mathrm{p}_{1},\left\|\boldsymbol{\sigma}^{D}\right\|\right) \boldsymbol{n}_{\mathbf{p}} \cdot \boldsymbol{\delta} \mathbf{p}+\Delta \sigma\|\boldsymbol{\delta} \mathbf{p}\|\right) \mathrm{d} x \geq 0
$$

To derive the criterion (35) in $\Omega_{1}^{-}$, the same proof by contradiction as in $\Omega_{\mathrm{p}}$ applies.

It remains to investigate the case of the subset $\Omega_{1}^{+}$. The vectorial space of deviatoric tensors can be decomposed as the direct sum of the vectorial space generated by $\mathbf{p}$ and its orthogonal part. The field $\boldsymbol{\sigma}^{D}-R\left(\mathrm{p}_{1},\left\|\boldsymbol{\sigma}^{D}\right\|\right) \boldsymbol{n}_{\mathbf{p}}$ is then written as $B \boldsymbol{n}_{\mathbf{p}}+\boldsymbol{C}_{\perp}$ with $B \in \mathbb{R}^{+}$and $\boldsymbol{C}_{\perp}=\operatorname{proj}_{\perp \mathbf{p}}\left(\boldsymbol{\sigma}^{D}-R\left(\mathrm{p}_{1},\left\|\boldsymbol{\sigma}^{D}\right\|\right) \boldsymbol{n}_{\mathbf{p}}\right)$ 
being the orthogonal projection to $\mathbf{p}$. Now let us suppose that $\left\|\boldsymbol{C}_{\perp}\right\|>\Delta \sigma$ on a subset $\Omega_{1}^{+*}$ of $\Omega_{1}^{+}$. We define the particular test direction $\delta \mathbf{p}^{*}$ as $\boldsymbol{C}_{\perp}$ normalized by its norm on $\Omega_{1}^{+*}$ and zero elsewhere. Such $\boldsymbol{\delta} \mathbf{p}^{*}$ is admissible by construction. Taking the scalar product between $\boldsymbol{\sigma}^{D}-R\left(\mathrm{p}_{1},\left\|\boldsymbol{\sigma}^{D}\right\|\right) \boldsymbol{n}_{\mathbf{p}}$ and $\boldsymbol{\delta} \mathbf{p}^{*}$, we find

$$
\left(\boldsymbol{\sigma}^{D}-R\left(\mathrm{p}_{1},\left\|\boldsymbol{\sigma}^{D}\right\|\right) \boldsymbol{n}_{\mathbf{p}}\right) \cdot \boldsymbol{\delta} \mathbf{p}^{*}=\left\|\boldsymbol{C}_{\perp}\right\|
$$

Then we deduce that

$$
\mathcal{E}^{\prime}(\boldsymbol{u}, \mathbf{p}, \overline{\mathbf{p}})\left(\mathbf{0}, \boldsymbol{\delta} \mathbf{p}^{*},\left\|\boldsymbol{\delta} \mathbf{p}^{*}\right\|\right)=\int_{\Omega_{1}^{+*}}\left(-\left\|\boldsymbol{C}_{\perp}\right\|+\Delta \sigma\right) \mathrm{d} x<0
$$

which is in contradiction with 42 .

From phase transformation criterion to first-order stability condition. It remains to prove that the phase transformation criteria are sufficient conditions for first-order stability. This task is achieved by making use of the Cauchy-Schwarz inequality on each subset $\Omega_{0}, \Omega_{\mathrm{p}}$ and $\Omega_{1}$. Given a test direction $\delta \mathbf{p} \in \mathcal{Z}_{0}$, we have, in virtue of the Cauchy-Schwarz inequality and the phase transformation criterion $(33)$ in $\Omega_{0}$, that

$$
\int_{\Omega_{0}}(\underbrace{-\boldsymbol{\sigma}^{D} \cdot \boldsymbol{\delta} \mathbf{p}}_{\geq-\left\|\boldsymbol{\sigma}^{D}\right\|\|\boldsymbol{\delta} \mathbf{p}\|}+\left(\Delta \sigma+R\left(0,\left\|\boldsymbol{\sigma}^{D}\right\|\right)\right)\|\boldsymbol{\delta} \mathbf{p}\|) \mathrm{d} x \geq 0
$$

The same argument applies in $\Omega_{\mathrm{p}}$ and $\Omega_{1}^{-}$. Let us notice in $\Omega_{1}^{+}$that

$$
\begin{aligned}
\int_{\Omega_{1}^{+}}\left(-\left(\boldsymbol{\sigma}^{D}-R\left(\|\mathbf{p}\|,\left\|\boldsymbol{\sigma}^{D}\right\|\right) \boldsymbol{n}_{\mathbf{p}}\right) \cdot \boldsymbol{\delta} \mathbf{p}+\Delta \sigma\|\boldsymbol{\delta} \mathbf{p}\|\right) \mathrm{d} x= \\
\int_{\Omega_{1}^{+}}\left(-\left(\left(\boldsymbol{\sigma}^{D}-R\left(\|\mathbf{p}\|,\left\|\boldsymbol{\sigma}^{D}\right\|\right) \boldsymbol{n}_{\mathbf{p}}\right) \cdot \boldsymbol{n}_{\mathbf{p}}\right)\left(\boldsymbol{n}_{\mathbf{p}} \cdot \boldsymbol{\delta} \mathbf{p}\right)\right. \\
\left.\quad-\operatorname{proj}_{\perp \mathbf{p}}\left(\boldsymbol{\sigma}^{D}-R\left(\|\mathbf{p}\|,\left\|\boldsymbol{\sigma}^{D}\right\|\right) \boldsymbol{n}_{\mathbf{p}}\right) \cdot \operatorname{proj}_{\perp \mathbf{p}}(\boldsymbol{\delta} \mathbf{p})+\Delta \sigma\|\boldsymbol{\delta} \mathbf{p}\|\right) \mathrm{d} x \geq 0
\end{aligned}
$$

Since $\|\mathbf{p}\|=\mathrm{p}_{1}$ in $\Omega_{1}^{+}$, we have $\boldsymbol{\delta} \mathbf{p} \cdot \boldsymbol{n}_{\mathbf{p}} \leq 0$. Moreover, we also have $\left(\boldsymbol{\sigma}^{D}-R\left(\|\mathbf{p}\|,\left\|\boldsymbol{\sigma}^{D}\right\|\right) \boldsymbol{n}_{\mathbf{p}}\right) \cdot \boldsymbol{n}_{\mathbf{p}} \geq 0$ in $\Omega_{1}^{+}$. Then we deduce, in virtue of criterion (36) and Cauchy-Schwarz inequality, that the right-hand side is necessarily positive. Finally, by summing each contribution in $\Omega_{0}, \Omega_{\mathrm{p}}, \Omega_{1}^{-}$and $\Omega_{1}^{+}$, in addition to the weak form of the mechanical equilibrium (38), we obtain the first-order stability condition (27).

The set of criteria given by (33)- 36 ) define a set of transformation domains in the deviatoric stress-space. To illustrate the shape of such domains, let us consider a model in dimension $N=2$ with $\mu: y \mapsto \mu_{0}$ being a material constant. Figures 2, 4 depict the transformation domains respectively for $\|\mathbf{p}\|=0,0<\|\mathbf{p}\|<\mathrm{p}_{1}$ and $\|\mathbf{p}\|=\mathrm{p}_{1}$ in the orthonormal basis $\left\{\boldsymbol{a}_{1}, \boldsymbol{a}_{2}\right\}$ of deviatoric space with

$$
\boldsymbol{a}_{1}=\frac{1}{\sqrt{2}}\left(\begin{array}{ll}
0 & 1 \\
1 & 0
\end{array}\right), \quad \boldsymbol{a}_{2}=\frac{1}{\sqrt{2}}\left(\begin{array}{cc}
1 & 0 \\
0 & -1
\end{array}\right)
$$




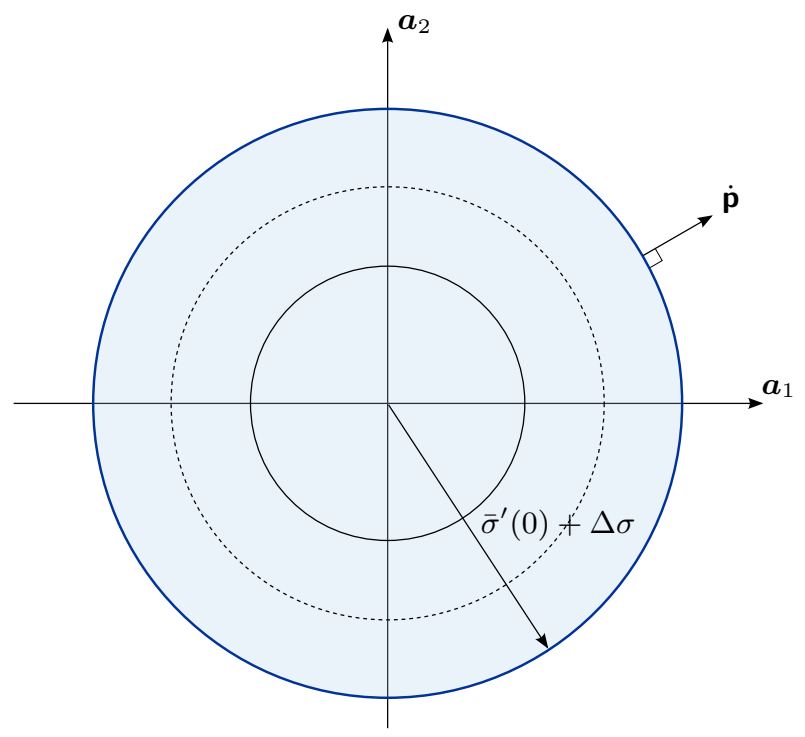

Figure 2: Transformation domain (blue area) in the deviatoric space $(N=2)$ for $\|\mathbf{p}\|=0$.

When the material is in its austenitic state with $\|\mathbf{p}\|=0$, the transformation domain is a disk centered at $(0,0)$ of radius $\bar{\sigma}^{\prime}(0)+\Delta \sigma$ (similar to the elastic domain of a Von-Mises plasticity model). However, as soon as the transformation is triggered with $0<\|\mathbf{p}\|<\mathrm{p}_{1}$, the transformation domain shifts to a smaller disk centered at the deviatoric stress $\bar{\sigma}(\|\mathbf{p}\|) \boldsymbol{n}_{\mathbf{p}}$ and of constant radius $\Delta \sigma$. As a result, the transformation domain moves when the direction of $\mathbf{p}$ evolves. When the martensitic transformation is complete with $\|\mathbf{p}\|=\mathrm{p}_{1}$, the transformation domain is unbounded in the direction $\boldsymbol{n}_{\mathbf{p}}$. Moreover, depending on the sign of $y \mapsto \bar{\sigma}^{\prime \prime}(y)$, transformation domains can move away $\left(\bar{\sigma}^{\prime \prime}>0\right)$ or get closer $\left(\bar{\sigma}^{\prime \prime}<0\right)$ to $\boldsymbol{\sigma}=\mathbf{0}$ as qualitatively depicted in Figure 11. Such stress-hardening and stress-softening behaviours have a strong impact on stability results, as investigated in Section 6 .

In addition to phase transformation criteria, consistency conditions must be supplied. In particular, those are fundamental to determine how transformation domains move in the deviatoric space when the direction of $\mathbf{p}$ evolves. These consistency conditions are not postulated but directly derived from the energy balance condition 25) and obey to a normality rule. Indeed we have

Proposition 4.4 (Consistency conditions). Providing regularity in time of an evolution path that satisfies the first-order stability conditions, the energy balance (25) holds if and only if the phase transformation rate $\dot{\mathbf{p}} \in \mathcal{Z}_{0}$ satisfies the following normality rules 


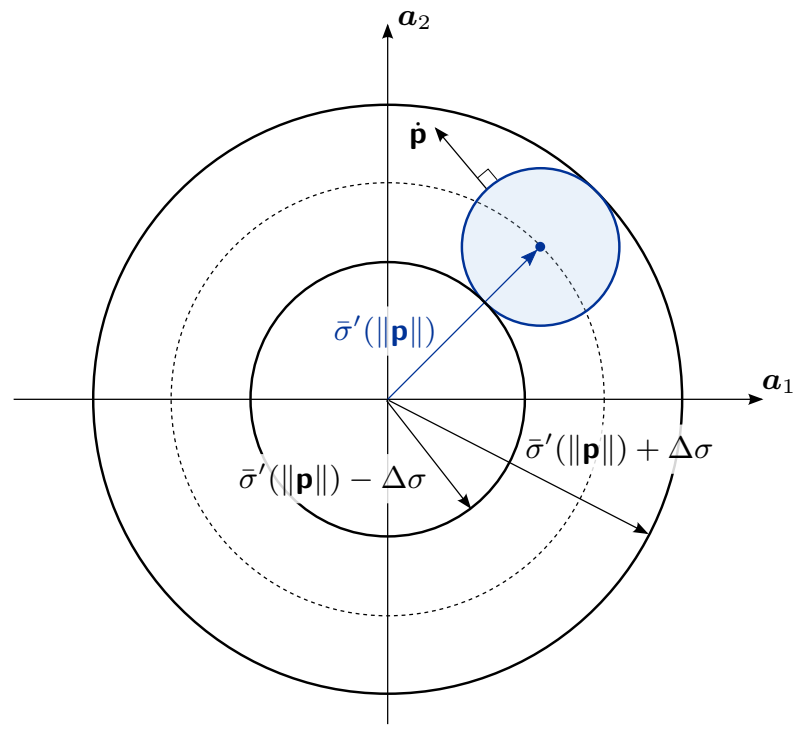

Figure 3: Transformation domain (blue area) in the deviatoric space $(N=2)$ for $0<\|\mathbf{p}\|<\mathbf{p}_{1}$ with constant $\mu$.

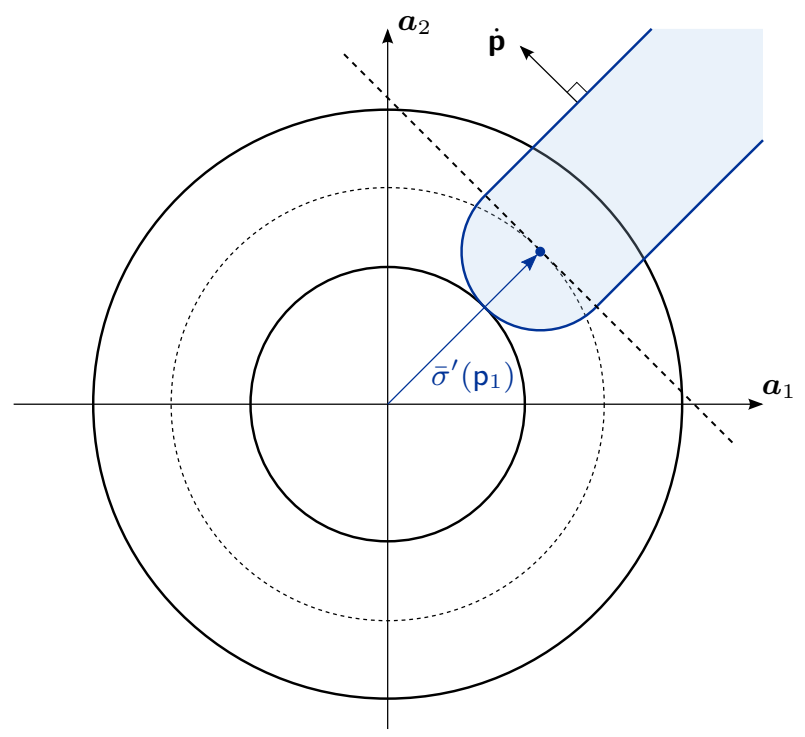

Figure 4: Transformation domain (blue area) in the deviatoric space $(N=2)$ for $\|\mathbf{p}\|=\mathrm{p}_{1}$ with constant $\mu$. 
- on the subset of $\Omega_{0}$ where $\|\mathbf{p}\|=0$ :

$$
\begin{cases}\left\|\boldsymbol{\sigma}^{D}\right\|=\Delta \sigma+R\left(0,\left\|\boldsymbol{\sigma}^{D}\right\|\right) & \Rightarrow \quad \exists \eta \geq 0, \quad \dot{\mathbf{p}}=\eta \boldsymbol{\sigma}^{D} \\ \left\|\boldsymbol{\sigma}^{D}\right\|<\Delta \sigma+R\left(0,\left\|\boldsymbol{\sigma}^{D}\right\|\right) & \Rightarrow \quad \dot{\mathbf{p}}=\mathbf{0}\end{cases}
$$

- on the subset of $\Omega_{\mathrm{p}}$ where $0<\|\mathbf{p}\|<\mathrm{p}_{1}$ :

$$
\left\{\begin{array}{lll}
\left\|\boldsymbol{\sigma}^{D}-R\left(\|\mathbf{p}\|,\left\|\boldsymbol{\sigma}^{D}\right\|\right) \boldsymbol{n}_{\mathbf{p}}\right\|=\Delta \sigma & \Rightarrow & \exists \eta \geq 0, \quad \dot{\mathbf{p}}=\eta\left(\boldsymbol{\sigma}^{D}-R\left(\|\mathbf{p}\|,\left\|\boldsymbol{\sigma}^{D}\right\|\right) \boldsymbol{n}_{\mathbf{p}}\right) \\
\left\|\boldsymbol{\sigma}^{D}-R\left(\|\mathbf{p}\|,\left\|\boldsymbol{\sigma}^{D}\right\|\right) \boldsymbol{n}_{\mathbf{p}}\right\|<\Delta \sigma & \Rightarrow & \dot{\mathbf{p}}=\mathbf{0}
\end{array}\right.
$$

- on the subset of $\Omega_{1}^{-}$where $\|\mathbf{p}\|=\mathrm{p}_{1}$ and $\mathbf{p} \cdot\left(\boldsymbol{\sigma}^{D}-R\left(\left\|\mathrm{p}_{1}\right\|,\left\|\boldsymbol{\sigma}^{D}\right\|\right) \boldsymbol{n}_{\mathbf{p}}\right)<0$ :

$$
\left\{\begin{array}{lll}
\left\|\boldsymbol{\sigma}^{D}-R\left(\mathrm{p}_{1},\left\|\boldsymbol{\sigma}^{D}\right\|\right) \boldsymbol{n}_{\mathbf{p}}\right\|=\Delta \sigma & \Rightarrow & \exists \eta \geq 0, \quad \dot{\mathbf{p}}=\eta\left(\boldsymbol{\sigma}^{D}-R\left(\mathrm{p}_{1},\left\|\boldsymbol{\sigma}^{D}\right\|\right) \boldsymbol{n}_{\mathbf{p}}\right) \\
\left\|\boldsymbol{\sigma}^{D}-R\left(\mathrm{p}_{1},\left\|\boldsymbol{\sigma}^{D}\right\|\right) \boldsymbol{n}_{\mathbf{p}}\right\|<\Delta \sigma & \Rightarrow & \dot{\mathbf{p}}=\mathbf{0}
\end{array}\right.
$$

- on the subset of $\Omega_{1}^{+}$where $\|\mathbf{p}\|=\mathrm{p}_{1}$ and $\mathbf{p} \cdot\left(\boldsymbol{\sigma}^{D}-R\left(\mathrm{p}_{1},\left\|\boldsymbol{\sigma}^{D}\right\|\right) \boldsymbol{n}_{\mathbf{p}}\right) \geq 0$ :

$$
\begin{cases}\left\|\operatorname{proj}_{\perp \mathbf{p}}\left(\boldsymbol{\sigma}^{D}-R\left(\mathrm{p}_{1},\left\|\boldsymbol{\sigma}^{D}\right\|\right) \boldsymbol{n}_{\mathbf{p}}\right)\right\|=\Delta \sigma & \Rightarrow \quad \exists \eta \geq 0, \quad \dot{\mathbf{p}}=\eta \operatorname{proj}_{\perp \mathbf{p}}\left(\boldsymbol{\sigma}^{D}-R\left(\mathrm{p}_{1},\left\|\boldsymbol{\sigma}^{D}\right\|\right) \boldsymbol{n}_{\mathbf{p}}\right), \\ \left\|\operatorname{proj}_{\perp \mathbf{p}}\left(\boldsymbol{\sigma}^{D}-R\left(\mathrm{p}_{1},\left\|\boldsymbol{\sigma}^{D}\right\|\right) \boldsymbol{n}_{\mathbf{p}}\right)\right\|<\Delta \sigma & \Rightarrow \quad \dot{\mathbf{p}}=\mathbf{0}\end{cases}
$$

Proof. First we assume that the energy balance holds. Taking the time derivative of (25), we find

$$
\frac{d \mathcal{E}}{d t}(\boldsymbol{u}, \mathbf{p}, \overline{\mathbf{p}})=\mathcal{E}^{\prime}(\boldsymbol{u}, \mathbf{p}, \overline{\mathbf{p}})(\dot{\boldsymbol{u}}, \dot{\mathbf{p}},\|\dot{\mathbf{p}}\|)=\int_{\partial_{D} \Omega}(\boldsymbol{\sigma} \cdot \mathbf{n}) \cdot \dot{U}_{\boldsymbol{d}} d x-\int_{\partial_{T} \Omega} \dot{\boldsymbol{T}}_{\boldsymbol{d}} \cdot \boldsymbol{u} d x
$$

With computations similar to (37) and making use of the mechanical equilibrium (32), as a necessary condition of first-order stability, we obtain

$$
\begin{aligned}
& 0=\int_{\Omega_{0}}\left(-\boldsymbol{\sigma}^{D} \cdot \dot{\mathbf{p}}+\left(\Delta \sigma+\bar{\sigma}^{\prime}(0)-\frac{1}{4}\left(\frac{1}{\mu}\right)^{\prime}(0)\left\|\boldsymbol{\sigma}^{D}\right\|^{2}\right)\|\dot{\mathbf{p}}\|\right) \mathrm{d} x \\
& +\int_{\Omega_{\mathrm{p}}}\left(-\left(\boldsymbol{\sigma}^{D}-R\left(\|\mathbf{p}\|,\left\|\boldsymbol{\sigma}^{D}\right\|\right) \boldsymbol{n}_{\mathbf{p}}\right) \cdot \dot{\mathbf{p}}+\Delta \sigma\|\dot{\mathbf{p}}\|\right) \mathrm{d} x \\
& +\int_{\Omega_{1}^{-}}\left(-\left(\boldsymbol{\sigma}^{D}-R\left(\mathrm{p}_{1},\left\|\boldsymbol{\sigma}^{D}\right\|\right) \boldsymbol{n}_{\mathbf{p}}\right) \cdot \dot{\mathbf{p}}+\Delta \sigma\|\dot{\mathbf{p}}\|\right) \mathrm{d} x \\
& +\int_{\Omega_{1}^{+}}\left(-\left(\boldsymbol{\sigma}^{D}-R\left(\mathrm{p}_{1},\left\|\boldsymbol{\sigma}^{D}\right\|\right) \boldsymbol{n}_{\mathbf{p}}\right) \cdot \dot{\mathbf{p}}+\Delta \sigma\|\dot{\mathbf{p}}\|\right) \mathrm{d} x
\end{aligned}
$$

Due to the phase transformation criteria (33), (34), (35) and as well as the Cauchy-Schwarz inequality, the integrand of each of the first three integrals of the right-hand side in 55 is positive. For the last integral 
over $\Omega_{1}^{+}$, given the equality

$$
\begin{aligned}
&-\left(\boldsymbol{\sigma}^{D}-R\left(\|\mathbf{p}\|,\left\|\boldsymbol{\sigma}^{D}\right\|\right) \boldsymbol{n}_{\mathbf{p}}\right) \cdot \dot{\mathbf{p}}+\Delta \sigma\|\dot{\mathbf{p}}\|= \\
&-\underbrace{\left(\left(\boldsymbol{\sigma}^{D}-R\left(\|\mathbf{p}\|,\left\|\boldsymbol{\sigma}^{D}\right\|\right) \boldsymbol{n} \mathbf{p}\right) \cdot \mathbf{p}\right)}_{\geq 0} \underbrace{(\mathbf{p} \cdot \dot{\mathbf{p}})}_{\leq 0}-\operatorname{proj}_{\perp \mathbf{p}}\left(\boldsymbol{\sigma}^{D}-R\left(\|\mathbf{p}\|,\left\|\boldsymbol{\sigma}^{D}\right\|\right) \boldsymbol{n}_{\mathbf{p}}\right) \cdot \dot{\mathbf{p}}+\Delta \sigma\|\dot{\mathbf{p}}\|
\end{aligned}
$$

the criterion (36) also ensures that the integrand of the last integral in the right-hand side of (55) is positive. As the sum of the four integrals has to be zero, we then deduce that the four positive integrands must necessarily be zero on their respective domain of integration. The nullity of each integrand in combination with the criteria (33)- 36 leads to a normality rule in each domain when $\dot{\mathbf{p}} \neq \mathbf{0}$ in virtue of the CauchySchwarz inequality.

Reciprocally, to retrieve the energy balance, it suffices to integrate the normality rules 50, 51, 53, and 52 over each domain respectively. By adding each contribution and making use of the mechanical equilibrium, the energy balance is reciprocally obtained.

In this Section, we have shown that first-order stability condition and energy balance are equivalent to a set of phase transformation criteria with appropriate consistency conditions. However, since the first-order stability condition is only a necessary condition of stability, a state might satisfy the phase transformation criteria without being directionally stable. Therefore, the second-order stability condition must be investigated. This task is accomplished in Section 6 after having discussed in the next section the mechanical properties of the model through some meaningful numerical examples.

\section{Numerical traction-torsion tests}

In this section we show some numerical results of the mechanical response of a thin-walled cylinder subjected to a combined three-dimensional traction-torsion test $([25,9])$ with the aim to provide more a qualitative illustration of the model behaviour rather than a quantitative comparison, which is out of scope of this work. Specifically, a non-proportional loading path is considered and different material models investigated, which describe both stress-hardening and stress-softening regimes. These analyses allow to understand and visualize not only the evolving and shifting transformation domain, predicted in Section 4 but also the orthogonality of the phase transformation strain rate with respect to the transformation domain. A full and exhaustive comparison with experiments is impossible due to the few experimental data concerning non-proportional loadings and the objective difficulty to characterize stress-softening behaviours. In this case, indeed, localizations are triggered and little information of the material response can be deduced from the structural response. Hence, only qualitative simulations are here considered with a homogeneous material response satisfying the first order stability condition and energy balance condition. The investigation and discussion of the second-order stability condition, which is rather technical and furnishes only upper and 
lower bounds for the three-dimensional traction-torsion experiment since it is associated to mixed forcedisplacement loadings, is postponed in the next section.

An engineering notation is here used where generic strain components are labeled as $\varepsilon_{i i} \rightarrow \varepsilon_{i}$ and $\varepsilon_{i j} \rightarrow \gamma_{i j} / 2$ when $i \neq j$, while the stress components as $\sigma_{i i} \rightarrow \sigma_{i}$ and $\sigma_{i j} \rightarrow \tau_{i j}$ when $i \neq j$. The problem is described through cylindrical coordinates $(r, \theta, z)$, due to the specific geometric configuration. The numerical simulations are strain driven by prescribing both $\varepsilon(t)=\varepsilon_{z}$ and $\gamma(t)=\gamma_{z \theta}=2 \varepsilon_{z \theta}$, with $t$ being the evolution time parameter. Moreover, accordingly to the boundary conditions of the traction-torsion test, the stress is such that $\sigma_{r}=\sigma_{z}=\tau_{r \theta}=\tau_{r z}=0$. Then, the general structure of the total strain, the phase transformation strain and the stress are equal, in matrix form, to

$$
\boldsymbol{\varepsilon}=\left(\begin{array}{ccc}
\varepsilon_{r} & 0 & 0 \\
0 & \varepsilon_{\theta} & \gamma(t) / 2 \\
0 & \gamma(t) / 2 & \varepsilon(t)
\end{array}\right), \quad \mathbf{p}=\left(\begin{array}{ccc}
-p_{z}-p_{\theta} & 0 & 0 \\
0 & p_{\theta} & p_{\theta z} \\
0 & p_{\theta z} & p_{z}
\end{array}\right), \quad \boldsymbol{\sigma}=\left(\begin{array}{ccc}
0 & 0 & 0 \\
0 & 0 & \tau \\
0 & \tau & \sigma
\end{array}\right)
$$

where $\sigma=\sigma_{z}, \tau=\tau_{\theta z}$, since no ambiguity can occur. The strain components $\varepsilon_{r}$ and $\varepsilon_{\theta}$ can be make explicit through the boundary conditions $\sigma_{r}=0, \sigma_{\theta}=0$ and the stress-strain law (31) as a function of the the phase transformation strain and the applied strains,

$$
\varepsilon_{r}=-\frac{3 K\left(p_{z}+2 p_{\theta}+\varepsilon\right)+2 \mu(\|\mathbf{p}\|)\left(2 p_{z}+p_{\theta}-\varepsilon\right)}{2(3 K+\mu(\|\mathbf{p}\|))} \quad \varepsilon_{\theta}=\frac{3 K\left(p_{z}+2 p_{\theta}-\varepsilon\right)-2 \mu(\|\mathbf{p}\|)\left(p_{z}-p_{\theta}-\varepsilon\right)}{2(3 K+\mu(\|\mathbf{p}\|))}
$$

Then, the only unknown of the problem remains the phase transformation strain $\mathbf{p}$.

With the aim of keeping the analysis simple, but sufficiently general, the constitutive functions in 19 . are chosen as

$$
\bar{\sigma}: y \mapsto \sqrt{\frac{2}{3}}\left(\bar{\sigma}_{0}\left(y-y^{2} / 2\right)+\bar{\sigma}_{1} y^{2} / 2\right)
$$

and

$$
\mu: y \mapsto \mu_{0}(1-y)+\mu_{1} y
$$

which are able to describe both hardening and softening behaviours as well as changes of the material stiffness. The constitutive parameters, which are common to all examined material models, are specified in Table 1. The remaining constitutive parameters, which are specific to the four different constitutive material models, namely the perfect model (PM), the hardening model (HM), the softening model 1 and 2 (SM1, SM2) are listed in Table 2. Furthermore, the material is initially assumed unstressed and purely austenitic, for all numerical simulations. 


\begin{tabular}{ll}
\hline$K$ & 1 \\
$\mu_{0}$ & $K / 2$ \\
$\bar{\sigma}_{0}$ & 0.75 \\
$\Delta \sigma$ & 0.20 \\
$\mathrm{p}_{1}$ & 1 \\
\hline
\end{tabular}

Table 1: Constitutive parameters common to all examined material models.

\begin{tabular}{lll}
\hline perfect model $(\mathrm{PM})$ & $\mu_{1}=\mu_{0}$ & $\bar{\sigma}_{1}=\bar{\sigma}_{0}$ \\
hardening model (HM) & $\mu_{1}=\mu_{0}$ & $\bar{\sigma}_{1}=1.2 \bar{\sigma}_{0}$ \\
softening model 1 (SM1) & $\mu_{1}=\mu_{0}$ & $\bar{\sigma}_{1}=0.8 \bar{\sigma}_{0}$ \\
& & \\
softening model 2 (SM2) & $\mu_{1}=\mu_{0} / 2$ & $\bar{\sigma}_{1}=0.8 \bar{\sigma}_{0}$ \\
\hline
\end{tabular}

Table 2: Examined material models with their specific material parameters.

\subsection{Non-Proportional loading material response}

The non-proportional (circular) strain loading path $t \mapsto(\varepsilon(t), \gamma(t))$, considered in this simulations, is represented in Figure 5a, accordingly to the experimental setup of [25], and is the same for the four compared material models of Table 2, To highlight and describe the material response of the present model, it is useful to introduce five stages in the strain space $(\varepsilon, \gamma / \sqrt{3})$, pointed out by the 5 (strain) state markers in Figure 5. O, A, B, C and D.

In stage OA, the material is elastically loaded in pure tension from the unstressed austenitic state O to the first transformation state A where the material reaches the boundary of the transformation domain, Figure 2, and the phase transformation is triggered. From state A, where the transformation domain reduces instantaneously its size as in Figure 3, to state B, the material continues to be radially loaded, i.e. stage AB. In this stage, the phase transformation strain increases on a fixed direction such that the norm of the phase transformation strain $\|\mathbf{p}\|$ stays below its critical value $\mathrm{p}_{1}$. Then, the material is enforced to follow a nonproportional circular strain path, i.e. stage BC, where the phase transformation strain mostly changes its orientation instead of its norm and the transformation domain turns around the origin accordingly to the phase transformation strain rate. At state $\mathrm{C}$, the material starts to be proportionally unloaded with the same initial radial strain loading direction until state D, where the transformation strain becomes again $\mathbf{0}$, 


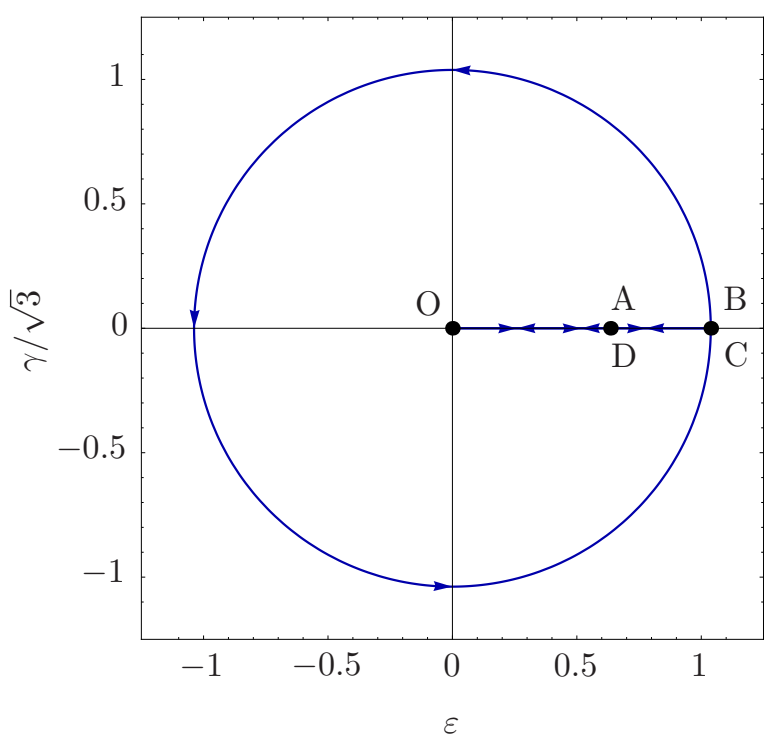

(a)

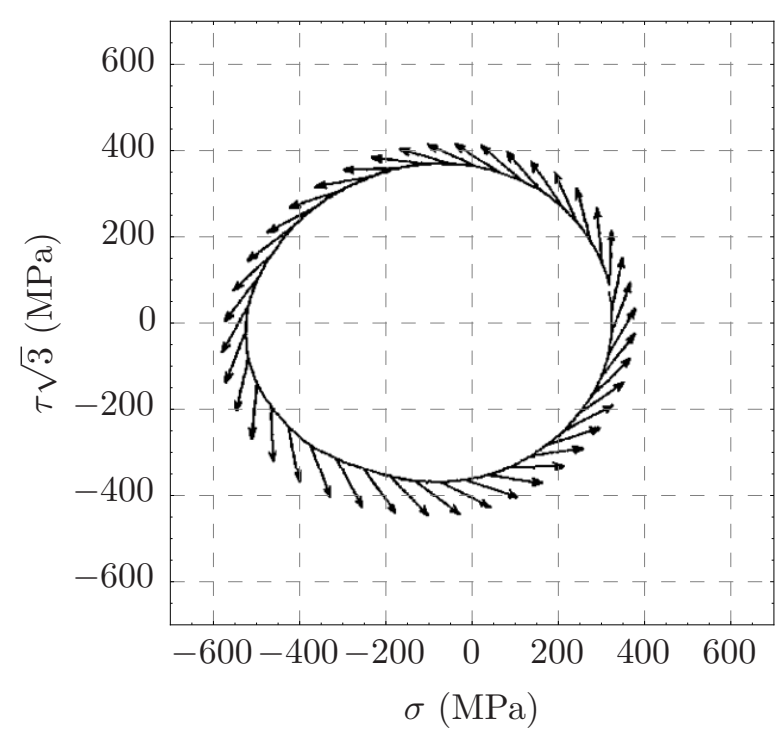

(b)

Figure 5: (a) The strain load path. (b) An experimental material response for a qualitatively equal circular strain loading path after some cycles, 25.

i.e. stage CD. The last stage DO corresponds to a simple elastic tensile radial unloading up to the initial unstressed and pure austenitic state.

The evolution problem of the material response is determined by an incremental minimization procedure. The strain load path is discretized in several steps. At each step the actual phase transformation strain is evaluated as the minimum of the total strain energy $(19)$. Such simple and straightforward solution procedure has been implemented within the software Mathematica, [4] taking full advantage of the built-in function NMinimize for constrained optimization.

The four material responses of Table 2 are represented in Figure 6, 7, 8, 9, and are described in their respective captions. Common features to all models are: (i) the shifting and turning of the transformation domain (gray closed paths) for $0<\|\mathbf{p}\|<\mathrm{p}_{1}$ accordingly to direction of the transformation strain rate and always inside an outer and inner transformation stress envelopes; (ii) the phase transformation strain rate following the normality rule as a result (black arrows); (iii) the distortion of the transformation domain from a disk for a non-constant $\mu$.

The hardening model (HM) in Figure $5 \mathrm{~b}$ is in good agreement with the experimental evidence of 25], although a precise comparison is impossible for several reasons as the material response of the experiment corresponds to a cycled material and is not enough detailed compared to our numerical simulation. 


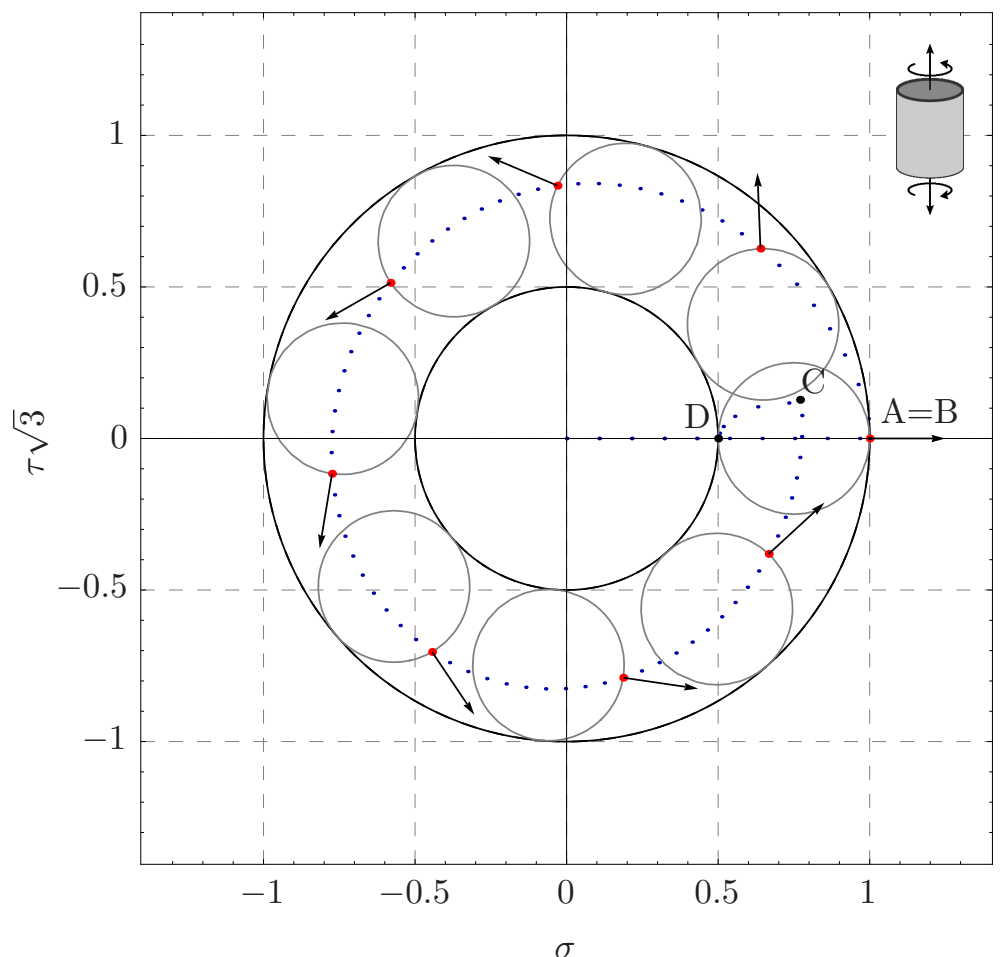

(a)

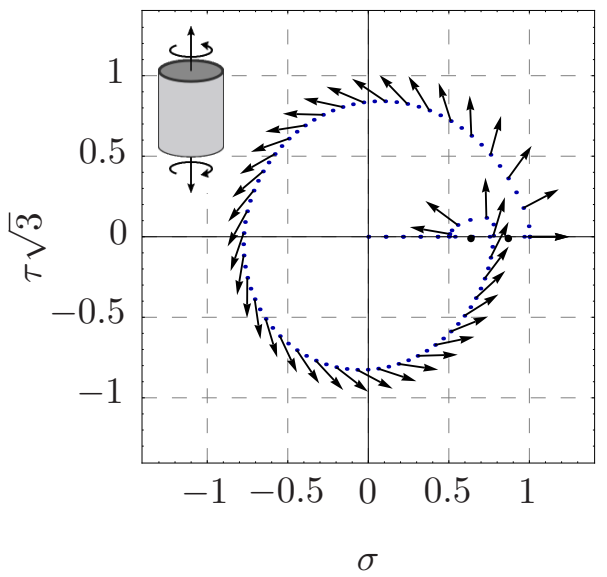

(b)

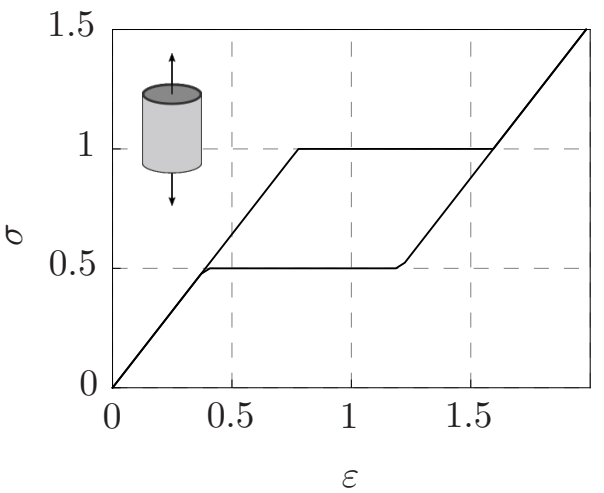

(c)

Figure 6: (a) Stress response of the perfect model (PM). The blue dots represent the stress response of the material point. Since $\bar{\sigma}^{\prime}$ and $\mu$ are constants, no hardening or softening occurs (indeed $\mathrm{A} \equiv \mathrm{B}$ ). The black circles represent the fixed inner and outer transformation stress envelopes for $0<\|\mathbf{p}\|<\mathrm{p}_{1}$. Some specific stress states are highlighted with red points. At each of these states, the corresponding evolving transformation domain is depicted in gray as well as the associated phase transformation strain rate direction, black arrow. (b) Phase transformation strain rate directions. (c) Stress response for the same material model for a proportional tensile loading-unloading strain path.

\subsection{Influence of $\mu(\|\mathbf{p}\|)$ on the transformation domain}

The aim of this section is to investigate the influence of the shear modulus $\mu(\|\mathbf{p}\|)$ on the transformation domain. More specifically, we wish to highlight only the role of the term $\mu^{\prime}$ in defining the shape of the transformation domain described by (34) in the stress space. For this purpose, first we only consider the softening model 2 (SM2) with a phase transformation strain that has just occurred, consequently a pure torsion test, namely $\mathbf{p}=\eta\left(\boldsymbol{a}_{\theta} \otimes \boldsymbol{a}_{z}+\boldsymbol{a}_{z} \otimes \boldsymbol{a}_{\theta}\right)$ with $0<\eta \ll 1$. Then, the first derivative of the shear modulus is left to vary with respect to the parameter $n \geq 0$ accordingly to the following law

$$
\mu: y \mapsto\left(\mu_{0}-\mu_{1}\right)\left(\mathrm{p}_{1}-y\right)^{n}+\mu_{1}
$$




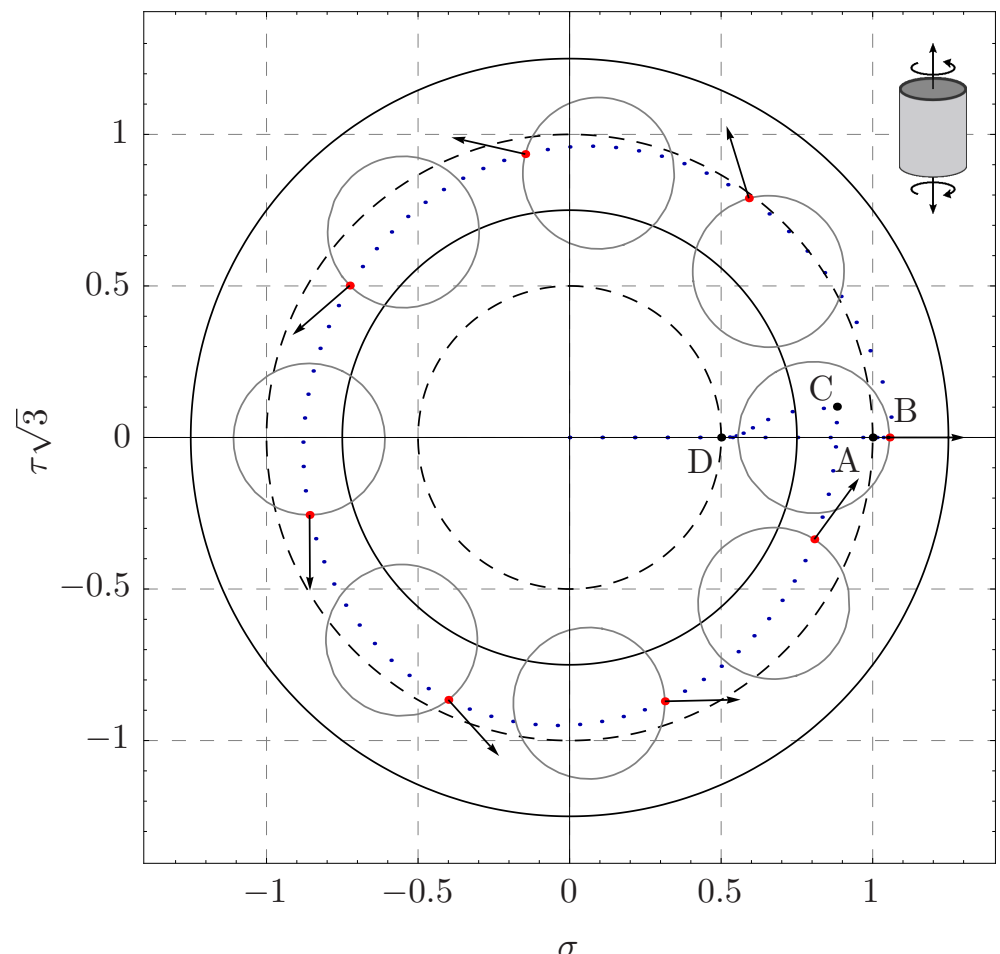

(a)

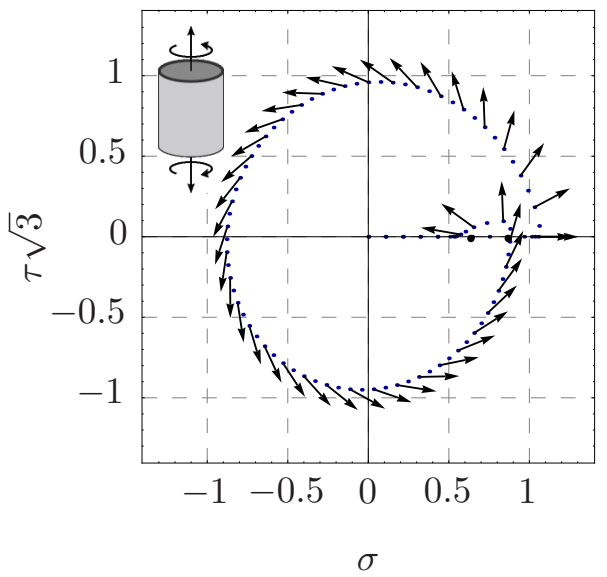

(b)

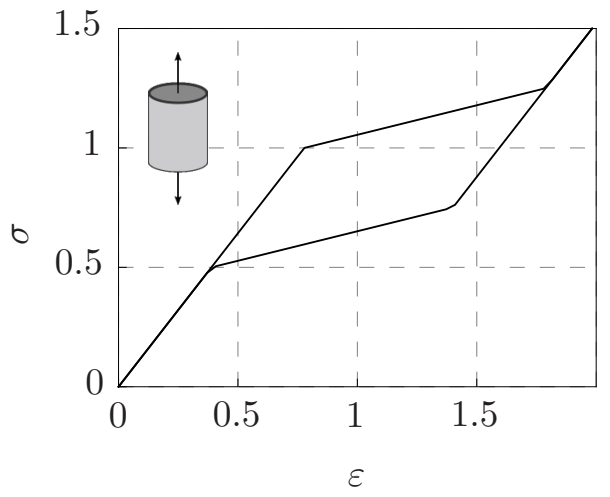

(c)

Figure 7: (a) Stress response of the hardening model (HM). The blue dots represent the stress response of the material point. Since $\bar{\sigma}^{\prime}$ is increasing, a hardening effect, in sense of Figure 11 is remarkable by the increasing stress level from state A to state B. Some specific stress states are highlighted with red points. At each of these states, the corresponding evolving transformation domain is depicted in gray as well as the associated phase transformation strain rate direction, black arrow. (b) Phase transformation strain rate directions. (c) Stress response for the same material model for a proportional tensile loading-unloading strain path with a hardening behaviour.

and the transformation domain derived accordingly to the phase transformation criterion. The main result of such analysis is shown in Figure 10. The initial round transformation domain, corresponding to $n=0$, tends to a non-convex domain with a shape of a lens by increasing $n$. Actually, the transformation domain remains always convex with respect to the driving force of the phase transformation. 


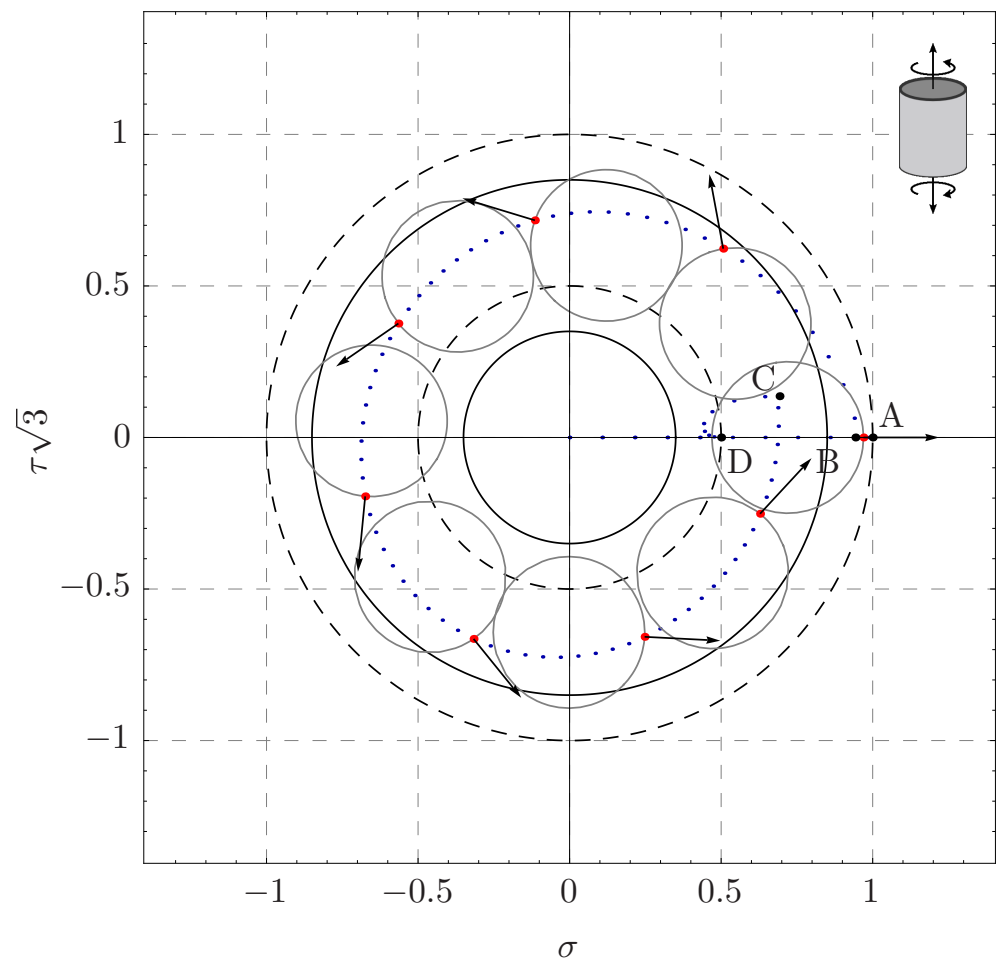

(a)

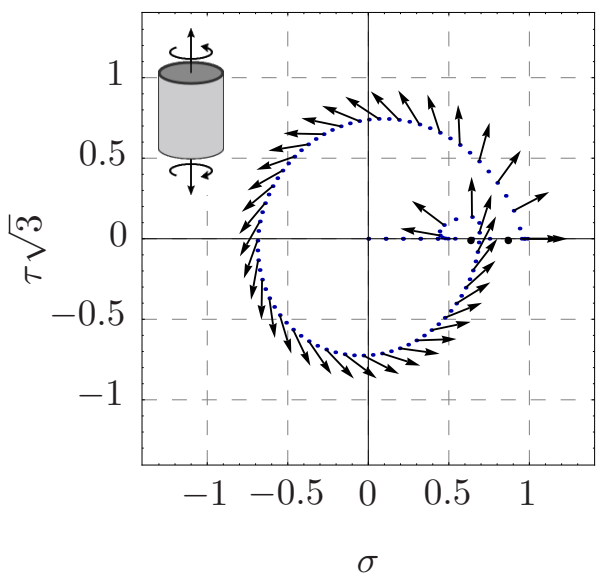

(b)

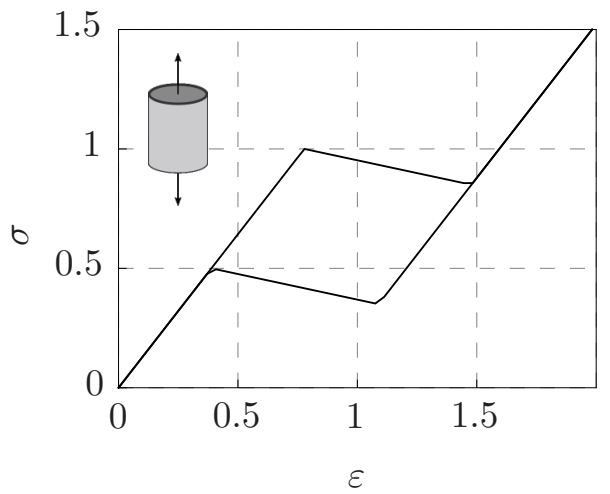

(c)

Figure 8: (a) Stress response of the softening model 1 (SM1). The blue dots represent the stress response of the material point. Since $\bar{\sigma}^{\prime}$ is decreasing, a softening effect, in sense of Figure 11 is remarkable by the decreasing stress level from state A to state B. Some specific stress states are highlighted with red points. At each of these states, the corresponding evolving transformation domain is depicted in gray as well as the associated phase transformation strain rate direction, black arrow. (b) Phase transformation strain rate directions. (c) Stress response for the same material model for a proportional tensile loading-unloading strain path with a softening behaviour.

\section{Stability analysis of homogeneous states}

\subsection{General statement}

We provide in this section a full stability analysis of homogeneous states. Those latter correspond to states for which the strain field $\varepsilon$, the phase transformation strain field $\mathbf{p}$ and hence the stress field $\boldsymbol{\sigma}$ are homogeneous in the body. As a result, the displacement $\boldsymbol{u}$ is linear and is equal to $\varepsilon x$ up to a rigid body motion. Such homogeneous states are of key importance in an identification procedure of a mechanical model and its material parameters as those states allow to directly link the material behavior to the global behavior of the body. Specifically, elastic properties e.g. Young modulus, Poisson ratio; material's yield strength or hardening parameters in plasticity models are identified from such homogeneous states. However, depending 


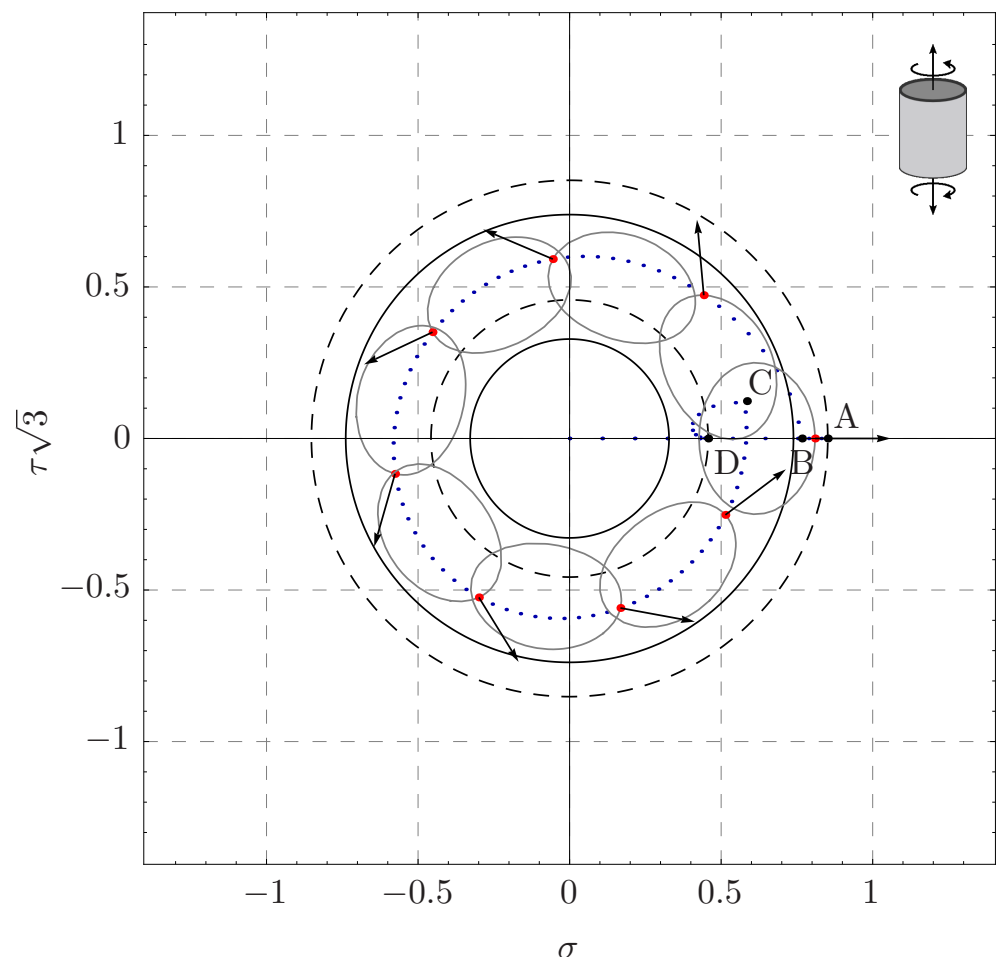

(a)

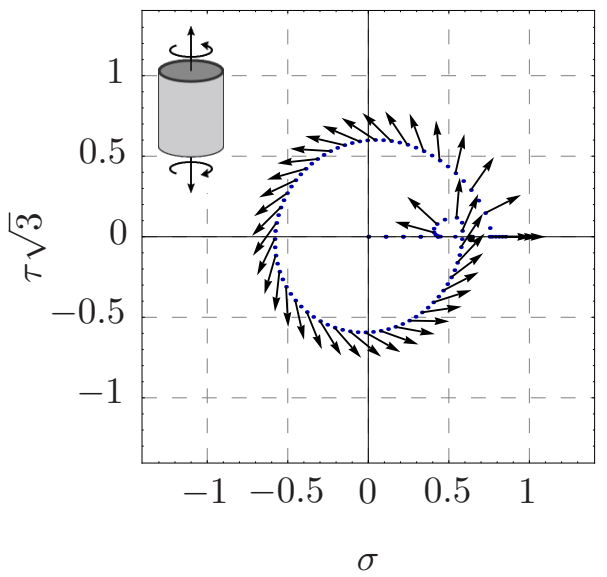

(b)

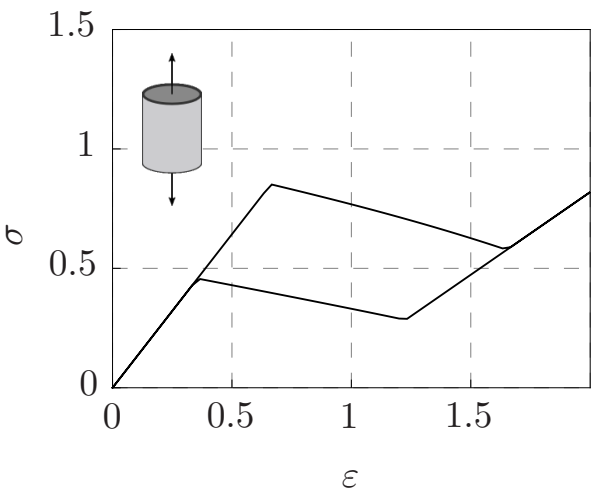

(c)

Figure 9: (a) Stress response of the softening model 2 (SM2). The blue dots represent the stress response of the material point. Since both $\bar{\sigma}^{\prime}$ and $\mu$ are decreasing with the increasing of the phase transformation strain norm, a softening effect, in sense of Figure 11 is remarkable by the decreasing stress level from state A to state B together with a distorted transformation domain, Section 5.2 Some specific stress states are highlighted with red points. At each of these states, the corresponding evolving transformation domain is depicted in gray as well as the associated phase transformation strain rate direction, black arrow. (b) Phase transformation strain rate directions. (c) Stress response for the same material model for a proportional tensile loading-unloading strain path with a softening behaviour and decreasing stiffness.

on the material behavior, observability of such states might not always be possible. For SMA such as NiTi under a classical uniaxial tension test, the elastic phase is usually followed by a localized evolution, hence making sensitive the use of such test for an appropriate identification of material parameters. Recently, Hallai and Kyriakydes have shown that such localized evolutions highlight an underlying stress-softening material behavior of the NiTi [17. Such experimental evidence is in accordance with recent one dimensional stability analysi: ${ }^{2}$ of softening SMA models in which stress-softening behavior is shown to lead to instability of a

\footnotetext{
${ }^{2}$ in terms of local minimality of the total energy
} 


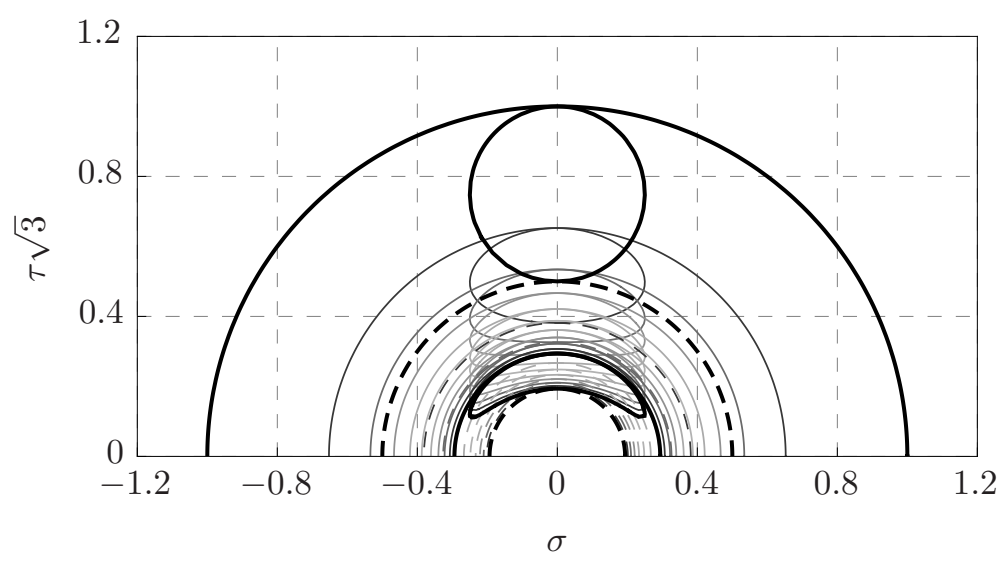

(a)

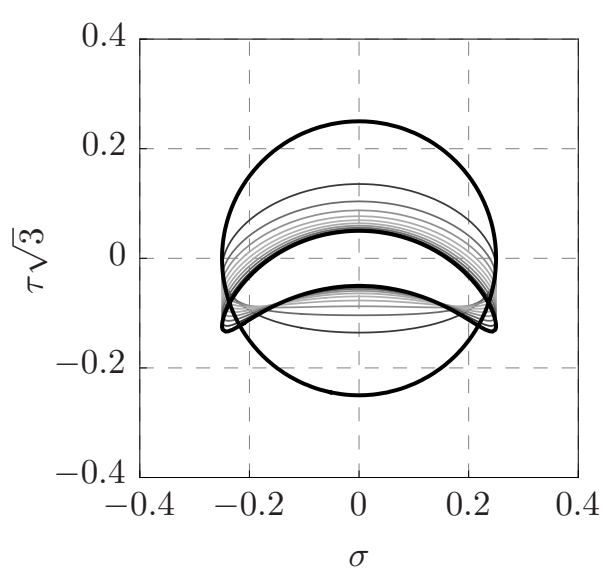

(b)

Figure 10: Influence of $\mu^{\prime}$ on the transformation domain accordingly to 61. (a) The round, thick, black domain corresponds to $n=0$ while the lenticular, thick, black domain to $n=5$. Transition curves from the limit domains are in gray. (b) The same domains shifted and centered to each other.

homogeneous phase-transformation [30, 1]. We propose in this section to expand such stability analysis to the three-dimensional case and investigate under which boundary conditions and for which material parameters a SMA model allows for stable and hence observable homogeneous states in view of model identification.

Let $(\boldsymbol{u}, \mathbf{p}, \overline{\mathbf{p}}) \in \mathcal{C} \times \mathcal{Z} \times \overline{\mathcal{Z}}$ be a homogeneous state in equilibrium ${ }^{3}$ and for which the phase transformation criterion is satisfied. According to Proposition 4.3 , the first-order derivative of the total energy of such state is then positive. The homogeneous state will be stable if the first-order derivative of the total energy is strictly positive i.e. $\mathcal{E}^{\prime}(\boldsymbol{u}, \mathbf{p}, \overline{\mathrm{p}})(\boldsymbol{\delta} \boldsymbol{u}, \boldsymbol{\delta} \mathbf{p},\|\boldsymbol{\delta} \mathbf{p}\|)>0$ for any test direction $(\boldsymbol{\delta} \boldsymbol{u}, \boldsymbol{\delta} \mathbf{p})$. Otherwise, if there exists a test direction $(\boldsymbol{\delta} \boldsymbol{u}, \boldsymbol{\delta} \mathbf{p})$ for which the first-order derivative is zero, then the sign of the second-order derivative must be investigated. Such situations are given by the following lemma

Lemma 6.1. For a homogeneous state $(\boldsymbol{u}, \mathbf{p}, \overline{\mathbf{p}}) \in \mathcal{C} \times \mathcal{Z} \times \overline{\mathcal{Z}}$ in equilibrium and satisfying the phasetransformation criterion, we have

$$
\mathcal{E}^{\prime}(\boldsymbol{u}, \mathbf{p}, \overline{\mathbf{p}})(\boldsymbol{\delta} \boldsymbol{u}, \boldsymbol{\delta} \mathbf{p},\|\boldsymbol{\delta} \mathbf{p}\|)=0
$$

if and only if a) $\boldsymbol{\delta} \mathbf{p}=\mathbf{0}$ when the corresponding phase transformation criterion is a strict inequality, b) there exists a positive scalar field $\eta: \Omega \mapsto \mathbb{R}^{+}$such that the following normality rules hold when the corresponding phase transformation criterion is an equality:

\footnotetext{
${ }^{3}$ with consistent homogeneous force or displacement prescribed at the boundary to ensure such equilibrium
} 
- when $\|\mathbf{p}\|=0$ and $\left\|\boldsymbol{\sigma}^{D}\right\|=\Delta \sigma+R\left(0,\left\|\boldsymbol{\sigma}^{D}\right\|\right)$ :

$$
\delta \mathbf{p}(x)=\eta(x) \boldsymbol{\sigma}^{D}
$$

- when $0<\|\mathbf{p}\|<\mathrm{p}_{1}$ and $\left\|\boldsymbol{\sigma}^{D}-R\left(\|\mathbf{p}\|,\left\|\boldsymbol{\sigma}^{D}\right\|\right) \boldsymbol{n}_{\mathbf{p}}\right\|=\Delta \sigma$ :

$$
\delta \mathbf{p}(x)=\eta(x)\left(\boldsymbol{\sigma}^{D}-R\left(\|\mathbf{p}\|,\left\|\boldsymbol{\sigma}^{D}\right\|\right) \boldsymbol{n}_{\mathbf{p}}\right)
$$

- when $\|\mathbf{p}\|=\mathrm{p}_{1}, \mathbf{p} \cdot\left(\boldsymbol{\sigma}^{D}-R\left(\left\|\mathrm{p}_{1}\right\|,\left\|\boldsymbol{\sigma}^{D}\right\|\right) \boldsymbol{n}_{\mathbf{p}}\right)<0$ and $\left\|\boldsymbol{\sigma}^{D}-R\left(\mathrm{p}_{1},\left\|\boldsymbol{\sigma}^{D}\right\|\right) \boldsymbol{n}_{\mathbf{p}}\right\|=\Delta \sigma$ :

$$
\boldsymbol{\delta} \mathbf{p}(x)=\eta(x)\left(\boldsymbol{\sigma}^{D}-R\left(\mathrm{p}_{1},\left\|\boldsymbol{\sigma}^{D}\right\|\right) \boldsymbol{n}_{\mathbf{p}}\right) ;
$$

- when $\|\mathbf{p}\|=\mathrm{p}_{1}, \mathbf{p} \cdot\left(\boldsymbol{\sigma}^{D}-R\left(\mathrm{p}_{1},\left\|\boldsymbol{\sigma}^{D}\right\|\right) \boldsymbol{n}_{\mathbf{p}}\right) \geq 0$ and $\left\|\operatorname{proj}_{\perp \mathbf{p}}\left(\boldsymbol{\sigma}^{D}-R\left(\mathrm{p}_{1},\left\|\boldsymbol{\sigma}^{D}\right\|\right) \boldsymbol{n}_{\mathbf{p}}\right)\right\|=\Delta \sigma$ :

$$
\delta \mathbf{p}(x)=\eta(x) \operatorname{proj}_{\perp \mathbf{p}}\left(\boldsymbol{\sigma}^{D}-R\left(\mathbf{p}_{1},\left\|\boldsymbol{\sigma}^{D}\right\|\right) \boldsymbol{n}_{\mathbf{p}}\right) .
$$

Proof. The proof is directly based on the proof of Proposition 4.4 as $(\boldsymbol{\delta} \mathbf{p},\|\boldsymbol{\delta} \mathbf{p}\|)$ play a similar role as $(\dot{\mathbf{p}},\|\dot{\mathbf{p}}\|)$ in (54).

\subsection{Stability of elastic states}

In the case where the phase transformation criterion is a strict inequality i.e. elastic states, the sign of the second-order derivative must be investigated only in the test direction $\boldsymbol{\delta} \mathbf{p}=\mathbf{0}$, see Lemma 6.1. It reads

$$
\mathcal{E}^{\prime \prime}(\boldsymbol{u}, \mathbf{p}, \overline{\mathbf{p}})(\boldsymbol{\delta} \boldsymbol{u}, \mathbf{0}, 0)=\int_{\Omega}\left(2 \mu(\|\mathbf{p}\|)\left\|\boldsymbol{\varepsilon}^{D}(\boldsymbol{\delta} \boldsymbol{u})\right\|^{2}+K(\operatorname{Tr}(\boldsymbol{\varepsilon}(\boldsymbol{\delta} \boldsymbol{u})))^{2}\right) \mathrm{d} x
$$

This is a positive-definitive quadratic form over $\mathcal{C}_{0}$ quotiented by the rigid body motions. Therefore, we deduce

Proposition 6.2. A homogeneous elastic state $(\boldsymbol{u}, \mathbf{p}, \overline{\mathrm{p}}) \in \mathcal{C} \times \mathcal{Z} \times \overline{\mathcal{Z}}$ in equilibrium is stable.

\subsection{Stability of phase-transforming states}

We now investigate the main case of the study which correspond to phase-transforming states for which the corresponding phase-transformation criterion is an equality. The sign of the second-order derivative must be evaluated only in test direction $\boldsymbol{\delta} \mathbf{p}$ satisfying the normality rule in this case, see Lemma 6.1. A direct calculation gives $4^{4}$

\footnotetext{
${ }^{4}$ we use the fact that up to the second-order in $h>0$, we have

$$
\|\mathbf{p}+h \boldsymbol{\delta} \mathbf{p}\|=\left\{\begin{array}{l}
h\|\boldsymbol{\delta} \mathbf{p}\| \quad \text { if } \mathbf{p}=\mathbf{0} \\
\|\mathbf{p}\|+h \frac{\mathbf{p} \cdot \boldsymbol{\delta} \mathbf{p}}{\|\mathbf{p}\|}+\frac{h^{2}}{2}\left(\frac{\|\boldsymbol{\delta} \mathbf{p}\|^{2}}{\|\mathbf{p}\|}-\frac{(\mathbf{p} \cdot \boldsymbol{\delta} \mathbf{p})^{2}}{\|\mathbf{p}\|^{3}}\right)+o\left(h^{2}\right) \quad \text { if } \mathbf{p} \neq \mathbf{0}
\end{array}\right.
$$
}


- if $\mathbf{p}=\mathbf{0}$ :

$$
\begin{aligned}
\mathcal{E}^{\prime \prime}(\boldsymbol{u}, \mathbf{p}, \overline{\mathbf{p}})(\boldsymbol{\delta} \boldsymbol{u}, \boldsymbol{\delta} \mathbf{p},\|\boldsymbol{\delta} \mathbf{p}\|)=\int_{\Omega}\left(2 \mu(\|\mathbf{p}\|)\left\|\boldsymbol{\varepsilon}^{D}(\boldsymbol{\delta} \boldsymbol{u})-\boldsymbol{\delta} \mathbf{p}\right\|^{2}+K(\operatorname{Tr}(\boldsymbol{\varepsilon}(\boldsymbol{\delta} \boldsymbol{u})))^{2}\right) \mathrm{d} x \\
\quad+\int_{\Omega}\left(2 \frac{\mu^{\prime}(0)}{\mu(0)} \boldsymbol{\sigma}^{D} \cdot\left(\varepsilon^{D}(\boldsymbol{\delta} \boldsymbol{u})-\boldsymbol{\delta} \mathbf{p}\right)\|\boldsymbol{\delta} \mathbf{p}\|+\left(\frac{\mu^{\prime \prime}(0)}{4(\mu(0))^{2}}\left\|\boldsymbol{\sigma}^{D}\right\|^{2}+\bar{\sigma}^{\prime \prime}(0)\right)\|\boldsymbol{\delta} \mathbf{p}\|^{2}\right) \mathrm{d} x
\end{aligned}
$$

- if $0<\|\mathbf{p}\| \leq \mathrm{p}_{1}$ :

$$
\begin{aligned}
& \mathcal{E}^{\prime \prime}(\boldsymbol{u}, \mathbf{p}, \overline{\mathbf{p}})(\boldsymbol{\delta} \boldsymbol{u}, \boldsymbol{\delta} \mathbf{p},\|\boldsymbol{\delta} \mathbf{p}\|)=\int_{\Omega}\left(2 \mu(\|\mathbf{p}\|)\left\|\boldsymbol{\varepsilon}^{D}(\boldsymbol{\delta} \boldsymbol{u})-\boldsymbol{\delta} \mathbf{p}\right\|^{2}+K(\operatorname{Tr}(\boldsymbol{\varepsilon}(\boldsymbol{\delta} \boldsymbol{u})))^{2}\right) \mathrm{d} x \\
& +\int_{\Omega}\left(2 \frac{\mu^{\prime}(\|\mathbf{p}\|)}{\mu(\|\mathbf{p}\|)} \boldsymbol{\sigma}^{D} \cdot\left(\varepsilon^{D}(\boldsymbol{\delta} \boldsymbol{u})-\boldsymbol{\delta} \mathbf{p}\right) \frac{\mathbf{p} \cdot \boldsymbol{\delta} \mathbf{p}}{\|\mathbf{p}\|}+\left(\frac{\mu^{\prime}(\|\mathbf{p}\|)}{4(\mu(\|\mathbf{p}\|))^{2}}\left\|\boldsymbol{\sigma}^{D}\right\|^{2}+\bar{\sigma}^{\prime}(\|\mathbf{p}\|)\right)\left(\frac{\|\boldsymbol{\delta} \mathbf{p}\|^{2}}{\|\mathbf{p}\|}-\frac{(\mathbf{p} \cdot \boldsymbol{\delta} \mathbf{p})^{2}}{\|\mathbf{p}\|^{3}}\right)\right. \\
& \left.+\left(\frac{\mu^{\prime \prime}(\|\mathbf{p}\|)}{4(\mu(\|\mathbf{p}\|))^{2}}\left\|\boldsymbol{\sigma}^{D}\right\|^{2}+\bar{\sigma}^{\prime \prime}(\|\mathbf{p}\|)\right) \frac{(\mathbf{p} \cdot \boldsymbol{\delta} \mathbf{p})^{2}}{\|\mathbf{p}\|^{2}}\right) \mathrm{d} x
\end{aligned}
$$

According to Lemma 6.1, the test direction $\boldsymbol{\delta} \mathbf{p}$ in which the second-order derivative of the total energy must satisfy the normality rule and hence be proportional to a fixed direction through a positive scalar field $\eta: \Omega \mapsto \mathbb{R}^{+}$. This leads to simplifications in the expression of $\mathcal{E}^{\prime \prime}(\boldsymbol{u}, \mathbf{p}, \overline{\mathbf{p}})(\boldsymbol{\delta} \boldsymbol{u}, \boldsymbol{\delta} \mathbf{p},\|\boldsymbol{\delta} \mathbf{p}\|)$ which becomes a bilinear function of $(\boldsymbol{u}, \eta)$ and which can be put under the following condensed form:

$$
\mathcal{E}^{\prime \prime}(\boldsymbol{u}, \mathbf{p}, \overline{\mathbf{p}})(\boldsymbol{\delta} \boldsymbol{u}, \boldsymbol{\delta} \mathbf{p},\|\boldsymbol{\delta} \mathbf{p}\|)=\int_{\Omega} \boldsymbol{A}(\boldsymbol{\varepsilon}(\boldsymbol{\delta} \boldsymbol{u})-\eta \boldsymbol{e}) \cdot(\boldsymbol{\varepsilon}(\boldsymbol{\delta} \boldsymbol{u})-\eta \boldsymbol{e}) \mathrm{d} x+\mathrm{G} \int_{\Omega} \eta^{2} \mathrm{~d} x
$$

where $\boldsymbol{A}, \boldsymbol{e}$ and $\mathrm{G}$ are respectively a positive definite homogeneous forth-order tensor, a homogeneous secondorder deviatoric tensor and a homogeneous scalar. All these quantities, which are functions of the current state $(\boldsymbol{u}, \mathbf{p}, \overline{\mathrm{p}})$, are given explicitly in the Appendix 2. Therefore, the study the sign of the second-order derivative consists in analyzing the following Rayleigh ratio defined over $\mathcal{C}_{0} \times L^{2}\left(\Omega, \mathbb{R}^{+}\right) \backslash(\mathbf{0}, 0)$ as

$$
\mathcal{R}:(\boldsymbol{u}, \eta) \mapsto \frac{\int_{\Omega} \boldsymbol{A}(\boldsymbol{\varepsilon}(\boldsymbol{\delta} \boldsymbol{u})-\eta \boldsymbol{e}) \cdot(\boldsymbol{\varepsilon}(\boldsymbol{\delta} \boldsymbol{u})-\eta \boldsymbol{e}) \mathrm{d} x}{\int_{\Omega} \eta^{2} \mathrm{~d} x} .
$$

and the stability of a phase-transforming homogeneous state is given by

Lemma 6.3. A homogeneous phase-transforming state $(\boldsymbol{u}, \mathbf{p}, \overline{\mathrm{p}}) \in \mathcal{C} \times \mathcal{Z} \times \overline{\mathcal{Z}}$ in equilibrium is stable if (resp. only if)

$$
\inf _{\mathcal{C}_{0} \times L^{2}\left(\Omega, \mathbb{R}^{+}\right) \backslash(\mathbf{0}, 0)} \mathcal{R}(\boldsymbol{u}, \eta)>(\text { resp. } \geq)-\mathrm{G} .
$$

We remark that the infimum of the Rayleigh ratio depends on the applied boundary conditions to ensure a homogeneous stress-strain field $(\boldsymbol{\sigma}, \boldsymbol{\varepsilon})$ as those boundary conditions define the admissible displacement space $\mathcal{C}_{0}$ over which the minimization is performed. We will explore successively the the following situations: homogeneous applied forces $\boldsymbol{\sigma} \mathbf{n}$ over $\partial \Omega$, homogeneous applied displacement $\boldsymbol{\varepsilon} x$ over $\partial \Omega$ and a combination of both. 


\subsubsection{Applied forces over $\partial \Omega$}

In this case, there is no constraint on the displacement test direction $\boldsymbol{\delta} \boldsymbol{u}$ on the boundary $\partial \Omega$ and the admissible displacement space reads

$$
\mathcal{C}_{0}=H^{1}\left(\Omega, \mathbb{R}^{n}\right)
$$

The minimization of the Rayleigh ratio over such space is straightforward and we obtain

Proposition 6.4. Under applied forces over $\partial \Omega$, a homogeneous phase transforming state in equilibrium is stable if (resp. only if)

$$
\text { G }>\text { (resp. } \geq) 0
$$

Proof. It suffices to remark that the infimum of the Rayleigh ratio is reached and is zero by taking the linear test function $\delta \boldsymbol{u}^{*}=\boldsymbol{e x}$ (hence $\boldsymbol{\varepsilon}(\boldsymbol{\delta} \boldsymbol{u})^{*}=\boldsymbol{e}$ ) and the test function $\eta^{*}=1$. Such arbitrary choice gives $\mathcal{R}\left(\boldsymbol{\delta} \boldsymbol{u}^{*}, \boldsymbol{e}\right)=0$ and thus, the stability of the state is given by the sign of the scalar G.

\subsubsection{Applied displacement over $\partial \Omega$}

In this case, as the displacement is constraint at the boundary $\partial \Omega$, then the admissible displacement space reads

$$
\mathcal{C}_{0}=\left\{\boldsymbol{u} \in H^{1}\left(\Omega, \mathbb{R}^{n}\right): \boldsymbol{u}=0 \text { on } \partial \Omega\right\} .
$$

The minimization of the Rayleigh ratio over such space in this case is not trivial and requires advanced results of calculus of variations. Such minimization of a similar Rayleigh ratio has been achieved in the context of damage by Pham and Marigo [32] based on a mathematical result established by Kohn [22] on quasi-convexification of a double well energy. Therefore, we encourage the interested reader to refer to these two references for a detailed proof of such minimization. Here, we only recall the main result

Proposition 6.5. Under applied displacement on $\partial \Omega$, a homogeneous phase transforming state in equilibrium is stable if (resp. only if)

$$
\boldsymbol{A} \boldsymbol{e} \cdot \boldsymbol{e}-\Gamma+\mathrm{G}>(\text { resp. } \geq) 0
$$

where $\Gamma$ is a positive scalar such that $\boldsymbol{A} \boldsymbol{e} \cdot \boldsymbol{e} \geq \Gamma$ and given by

$$
\Gamma=\max _{\|\boldsymbol{k}\|=1} \boldsymbol{A} \boldsymbol{\xi}(\boldsymbol{k}) \cdot \boldsymbol{\xi}(\boldsymbol{k})
$$

with $\boldsymbol{\xi}(\boldsymbol{k})$ being defined as

$$
\boldsymbol{\xi}(\boldsymbol{k})=\underset{\boldsymbol{w} \in \mathbb{V}(\boldsymbol{k})}{\operatorname{argmin}} \boldsymbol{A}(\boldsymbol{e}-\boldsymbol{w}) \cdot(\boldsymbol{e}-\boldsymbol{w})
$$

where $\mathbb{V}(\boldsymbol{k})$ is the space of compatible tensors generated by $\boldsymbol{k} \in \mathbb{R}^{n}$ i.e.

$$
\mathbb{V}(\boldsymbol{k})=\left\{\boldsymbol{w} \otimes \boldsymbol{k}+\boldsymbol{k} \otimes \boldsymbol{w}, \quad \boldsymbol{w} \in \mathbb{R}^{n}\right\}
$$


Proof. It consists in proving that the infimum of the Rayleigh ratio is given by

$$
\inf _{\mathcal{C}_{0} \times L^{2}\left(\Omega, \mathbb{R}^{+}\right) \backslash(0,0)} \mathcal{R}(\boldsymbol{u}, \eta)=\boldsymbol{A} \boldsymbol{e} \cdot \boldsymbol{e}-\Gamma
$$

Thus, we invite the reader to refer to Lemma 1 in 32 for a sketch of the proof based on Kohn's result $22]$.

As a result, compared to the previous case of prescribed forces on the boundary, the stability under prescribed displacement involves the additional calculation of a parameter $\Gamma$ which only depends on the stiffness tensor. In a general context where $\boldsymbol{A}$ can be anisotropic, no explicit value can be found to our knowledge. However, for the limiting case of isotropic constitutive law $\boldsymbol{A}$, which is precisely our case of interest, Kohn established in [22] an explicit procedure to obtain its value. We recall here briefly the result.

Identification of $\Gamma$. Starting from Lemma 4.3 in [22, the minimizer $\boldsymbol{\xi}(\boldsymbol{k})$ in 278 can be explicitly computed. Then, (77) can be simplified as

$$
\Gamma=\mu(\|\mathbf{p}\|) \max _{\|\boldsymbol{k}\|=1} F(\boldsymbol{k})
$$

with the function $F: \mathbb{M}_{s}^{N} \mapsto \mathbb{R}$ given by

$$
F: \boldsymbol{k} \mapsto 4\left(\|\boldsymbol{e} \boldsymbol{k}\|^{2}-(\boldsymbol{e} \boldsymbol{k} \cdot \boldsymbol{k})^{2}\right)+\frac{4}{2+\gamma}(\boldsymbol{e} \boldsymbol{k} \cdot \boldsymbol{k})^{2}, \quad \gamma=\frac{N K-2 \mu(\|\mathbf{p}\|)}{N \mu(\|\mathbf{p}\|)}
$$

The maximization of 81 is achieved upon the introduction of a Lagrange multiplier. Specifically, the maximizer $\boldsymbol{k}$ is among the states that satisfy the first variation of 81) i.e. for which there exists a $\lambda$ such that

$$
F^{\prime}(\boldsymbol{k})=\lambda \boldsymbol{k} \quad \Leftrightarrow \quad \boldsymbol{e}^{2} \boldsymbol{k}+\beta \boldsymbol{e} \boldsymbol{k}=\lambda \boldsymbol{k}
$$

with

$$
\beta=-\left(1+\frac{\gamma}{2+\gamma}\right) \boldsymbol{e} \boldsymbol{k} \cdot \boldsymbol{k}
$$

Calling respectively $\left(e_{i}\right)_{1 \leq i \leq N}$ and $\left(\boldsymbol{e}_{i}^{*}\right)_{1 \leq i \leq N}$ the eigenvalues and the eigenvectors of the symmetric tensor $\boldsymbol{e}$ and rewriting 83 in the eigenspace with $\boldsymbol{k}=\sum_{i=1}^{N} k_{i} \boldsymbol{e}_{i}^{*}$ gives

$$
e_{i}^{2}+\beta e_{i}=\lambda \quad \text { or } \quad k_{i}=0, \quad 1 \leq i \leq N
$$

For a given couple $(\beta, \lambda)$, the polynomial equation of degree two $X^{2}+\beta X-\lambda=0$ can have at most two distinct solutions. Therefore, as all the eigenvalues of $\boldsymbol{e}$ must satisfy that polynomial equation, one deduces that two situations can only occur: i) either $\boldsymbol{k}$ is an eigenvector of $\boldsymbol{e}$ or ii) $\boldsymbol{k}$ is a linear combination of two eigenvectors with two distinct eigenvalues.

- Case $i$ ): If $\boldsymbol{k}$ is an eigenvector of $\boldsymbol{e}$ associated to an eigenvalue $e_{i}$, then the value $F(\boldsymbol{k})$ is directly deduced from 82 and reads

$$
F(\boldsymbol{k})=\frac{4 e_{i}^{2}}{2+\gamma}
$$


- Case $i$ i): Otherwise $\boldsymbol{k}$ is a linear combination of two eigenvectors of $\boldsymbol{e}$, say $\left(\boldsymbol{e}_{1}^{*}, \boldsymbol{e}_{2}^{*}\right)$ of eigenvalues $\left(e_{1}, e_{2}\right)$ with $e_{1}<e_{2}$, that satisfies the following conditions

$$
\boldsymbol{k}=\boldsymbol{e}_{1}^{*}+\boldsymbol{e}_{2}^{*}, \quad\left\|\boldsymbol{e}_{1}^{*}\right\|^{2}+\left\|\boldsymbol{e}_{2}^{*}\right\|^{2}=1, \quad\left\|\boldsymbol{e}_{1}^{*}\right\|>0, \quad\left\|\boldsymbol{e}_{2}^{*}\right\|>0
$$

As $e_{1}$ and $e_{2}$ solves $X^{2}+\beta X-\lambda=0$, we have necessarily $\beta=-\left(e_{1}+e_{2}\right)$ and $\lambda=-e_{1} e_{2}$. From 84, one then obtains

$$
\boldsymbol{e} \boldsymbol{k} \cdot \boldsymbol{k}=e_{1}\left\|\boldsymbol{e}_{1}^{*}\right\|^{2}+e_{2}\left\|\boldsymbol{e}_{2}^{*}\right\|^{2}=\frac{\left(e_{1}+e_{2}\right)(2+\gamma)}{2(1+\gamma)}
$$

The system (87) and 88 admits a solution if and only if

$$
e_{1}<\frac{\left(e_{1}+e_{2}\right)(2+\gamma)}{2(1+\gamma)}<e_{2}
$$

Provided that 89 holds, taking the scalar product between 83 and $\boldsymbol{k}$ gives

$$
\|\boldsymbol{e} \boldsymbol{k}\|^{2}=-e_{1} e_{2}+\left(e_{1}+e_{2}\right) \boldsymbol{e} \boldsymbol{k} \cdot \boldsymbol{k}
$$

Combining (88) and (90) allows the computation of $F(\boldsymbol{k})$ for this case.

$\Gamma$ is finally obtained by taking the largest value of $F(\boldsymbol{k})$ among all the possibilities given by Case $i$ ) and Case ii) through the Kohn's procedure.

Remark 3. In the two-dimensional case $N=2$, the deviatoric tensor $\boldsymbol{e}$ has two eigenvalues $\left(e_{1}, e_{2}\right)$ with $e_{1}=-e_{2}$. While the Case i) leads to $F(\boldsymbol{k})=4 e_{1}^{2} /(2+\gamma)$, Case ii) gives $F(\boldsymbol{k})=4 e_{1}^{2}>4 e_{1}^{2} /(2+\gamma)$. As a result we have $\Gamma=4 \mu e_{1}^{2}=A \boldsymbol{e} \cdot \boldsymbol{e}$. We deduce that for $N=2$ the minimization of the Rayleigh ration gives $\inf _{\mathcal{C}_{0} \times L^{2}\left(\Omega, \mathbb{R}^{+}\right) \backslash(\mathbf{0}, 0)} \mathcal{R}(\boldsymbol{u}, \eta)=0$. As a result, the stability under prescribed displacement on $\partial \Omega$ is identical as under prescribed forces and hence is given by the sign of $\mathrm{G}$.

\subsubsection{Combined applied forces and displacements over $\partial \Omega$.}

In the general case where a part of the boundary $\partial_{D} \Omega$ is submitted to prescribed displacements and the remaining part $\partial_{T} \Omega$ is submitted to prescribed forces, the infimum of the Rayleigh ratio cannot be computed explicitly to our knowledge for any situation. However, upper and lower bounds can be found based on the two previous cases. Indeed, the admissible displacement space $\mathcal{C}_{0}$ always satisfies in this general case the following inequalities in terms of subsets

$$
H^{1}\left(\Omega, \mathbb{R}^{n}\right) \subset \mathcal{C}_{0} \subset H_{0}^{1}\left(\Omega, \mathbb{R}^{n}\right)
$$

where $H^{1}\left(\Omega, \mathbb{R}^{n}\right)$ and $H_{0}^{1}\left(\Omega, \mathbb{R}^{n}\right)$ correspond to the admissible displacement spaces for prescribed forces and prescribed displacement over $\partial \Omega$ respectively. Therefore, the previous cases can be used as lower and upper bounds for the minimizer of the Rayleigh ratio and we find

$$
\mathrm{G} \leq \inf _{\mathcal{C}_{0} \times L^{2}\left(\Omega, \mathbb{R}^{+}\right) \backslash(\mathbf{0}, 0)} \mathcal{R}(\boldsymbol{u}, \eta)+\mathrm{G} \leq \boldsymbol{A} \boldsymbol{e} \cdot \boldsymbol{e}-\Gamma+\mathrm{G}
$$

As a result, we obtain the following stability criterion for homogeneous states 
Proposition 6.6. Under mixed boundary conditions over $\partial \Omega$, a homogeneous phase transforming state in equilibrium is stable if $\mathrm{G}>0$ and unstable if $\boldsymbol{A} \boldsymbol{e} \cdot \boldsymbol{e}-\Gamma+\mathrm{G}<0$.

Remark 4. In the two-dimensional case, we know from Remark 3 that the infimum of the Rayleigh ratio under prescribed forces over $\partial \Omega$ or prescribed displacements over $\partial \Omega$ is zero. Then, a straightforward application of Proposition 6.6 in dimension $N=2$ shows that a homogeneous phase-transforming state in equilibrium is directionally stable under any kind of combined applied forces and displacements over $\partial \Omega$ if (resp. only if) $\mathrm{G}>($ resp. $\geq) 0$.

\subsection{Examples}

We illustrate here the stability results with a specific phase-transformation law for which $\mu: y \mapsto \mu_{0}$ is a material constant, leaving $\bar{\sigma}: y \mapsto \bar{\sigma}(y)$ as the only material function. For such law, a uniaxial tension test exhibits stress-hardening or stress-softening depending respectively if $\bar{\sigma}^{\prime \prime}>0$ or $\bar{\sigma}^{\prime \prime}<0$, see Figure 11 . The goal is to show that, despite stress-softening, it is possible to stabilize homogeneous phase-transforming states and avoid localized states. From Proposition 6.2, we know that elastic states are unconditionally stable so we only have to look at the stability of phase-transforming states for which the transformation criterion is an equality.

Stress-hardening: $\bar{\sigma}^{\prime \prime}(\|\mathbf{p}\|)>0$. In this case, one can immediately deduce from 110)-111] that $G>0$ when $\bar{\sigma}^{\prime \prime}>0$. As a result, given Proposition 6.6. homogeneous phase-transforming state will always be unconditionally stable in this case, regarding the kind of applied boundary conditions.

Stress-softening: $\bar{\sigma}^{\prime \prime}(\|\mathbf{p}\|)<0$. For sake of simplicity, we limit our study to dimension $N=2$ for which the stability of the state depends only on the sign of $\mathrm{G}$ and not on the kind of applied boundary conditions (force, displacement or mixed), see Remark 4

First, suppose that $\|\mathbf{p}\|=0$, then a calculation shows that $G=(\Delta \sigma)^{2} \bar{\sigma}^{\prime \prime}(0)<0$ and thus, the homogeneous state is unstable.

Now suppose that $0<\|\mathbf{p}\|<\mathbf{p}_{1}$. In virtue of the phase-transformation criterion, we have $\left\|\boldsymbol{\sigma}^{D}-\bar{\sigma}^{\prime}(\|\mathbf{p}\|) \boldsymbol{n}_{\mathbf{p}}\right\|=$ $\Delta \sigma$. Let us introduce the angle $\theta \in[0, \pi]$ between $\boldsymbol{\sigma}^{D}-\bar{\sigma}^{\prime}(\|\mathbf{p}\|) \boldsymbol{n}_{\mathbf{p}}$ and $\boldsymbol{n}_{\mathbf{p}}$ such that

$$
\cos \theta=\frac{\left(\boldsymbol{\sigma}^{D}-\bar{\sigma}^{\prime}(\|\mathbf{p}\|) \boldsymbol{n}_{\mathbf{p}}\right) \cdot \boldsymbol{n}_{\mathbf{p}}}{\Delta \sigma}
$$

A direct computation of $\mathrm{G}$ gives

$$
\frac{\mathrm{G}}{(\Delta \sigma)^{2}}=\frac{\bar{\sigma}^{\prime}(\|\mathbf{p}\|)}{\|\mathbf{p}\|}\left(1-(\cos \theta)^{2}\right)+\bar{\sigma}^{\prime \prime}(\|\mathbf{p}\|)(\cos \theta)^{2} .
$$

Therefore, we deduce that the homogeneous state will be stable for $0<\|\mathbf{p}\|<\mathrm{p}_{1}$ if (resp. only if) $\theta$ is such that

$$
|\cos \theta|<(\text { resp. } \leq) \cos \theta_{c}
$$


with $\theta_{c} \in\left[0, \frac{\pi}{2}\right]$ being a critical stability angle defined as

$$
\theta_{c}=\arccos \left(\sqrt{\frac{\bar{\sigma}^{\prime}(\|\mathbf{p}\|)}{\bar{\sigma}^{\prime}(\|\mathbf{p}\|)-\bar{\sigma}^{\prime \prime}(\|\mathbf{p}\|)\|\mathbf{p}\|}}\right)
$$

Finally suppose that $\|\mathbf{p}\|=\mathbf{p}_{1}$. On the one hand, if $\left(\boldsymbol{\sigma}^{D}-\bar{\sigma}^{\prime}(\|\mathbf{p}\|) \boldsymbol{n}_{\mathbf{p}}\right) \cdot \boldsymbol{n}_{\mathbf{p}} \geq 0$, then the normality rule 66 gives $\delta \mathbf{p} \cdot \mathbf{p}=0$. Therefore, $G=\bar{\sigma}^{\prime}(\|\mathbf{p}\|) \frac{|\Delta \sigma|}{\|\mathbf{p}\|}>0$ and the state is stable. On the other hand, if $\left(\boldsymbol{\sigma}^{D}-\bar{\sigma}^{\prime}(\|\mathbf{p}\|) \boldsymbol{n}_{\mathbf{p}}\right) \cdot \boldsymbol{n}_{\mathbf{p}}<0$, by analogy with the case $0<\|\mathbf{p}\|<\mathrm{p}_{1}$, the state will be stable if (resp. only if) 95) holds with $\|\mathbf{p}\|=\mathrm{p}_{1}$.

Figure 12 gives a geometric interpretation of such stability results in the deviatoric space. Blue lines and blue areas correspond to stable states while red lines correspond to unstable states. Such stability analysis gives some interesting perspectives on how to ensure homogeneous transformation in stress-softening SMA in view of a more consistent identification of the material parameters. First our stability analysis confirms that uniaxial tension experiments lead to inhomogeneous phase-transformation. Indeed, in a uniaxial test, the transformation strain $\mathbf{p}$ and the deviatoric stress $\boldsymbol{\sigma}^{D}$ evolve in such a way that they stay collinear to a fixed direction $\boldsymbol{n}_{\mathbf{p}}$. As a result $\boldsymbol{\sigma}^{D}$ will always stay on the red line in the deviatoric space on Figure 12 and the homogeneous state will be unstable. However, Figure 12 also suggests the existence of alternative evolution path to overcome such unstability issues. By changing the loading direction so that, in the deviatoric stress space, $\sigma^{D}$ always stays on the blue line delimiting the stable area, the homogeneous state will be stable. Such non-proportional loading will change the direction of $\dot{\mathbf{p}}$, hence changing the direction $\boldsymbol{n}_{\mathbf{p}}$ and shifting the transformation domain. Provided that $\left(\boldsymbol{\sigma}^{D}-\bar{\sigma}^{\prime}(\|\mathbf{p}\|) \boldsymbol{n}_{\mathbf{p}}\right) \cdot \boldsymbol{n}_{\mathbf{p}}>0$, such loading path will increase $\|\mathbf{p}\|$ until it reaches $\mathrm{p}_{1}$, hence completing the transformation. 

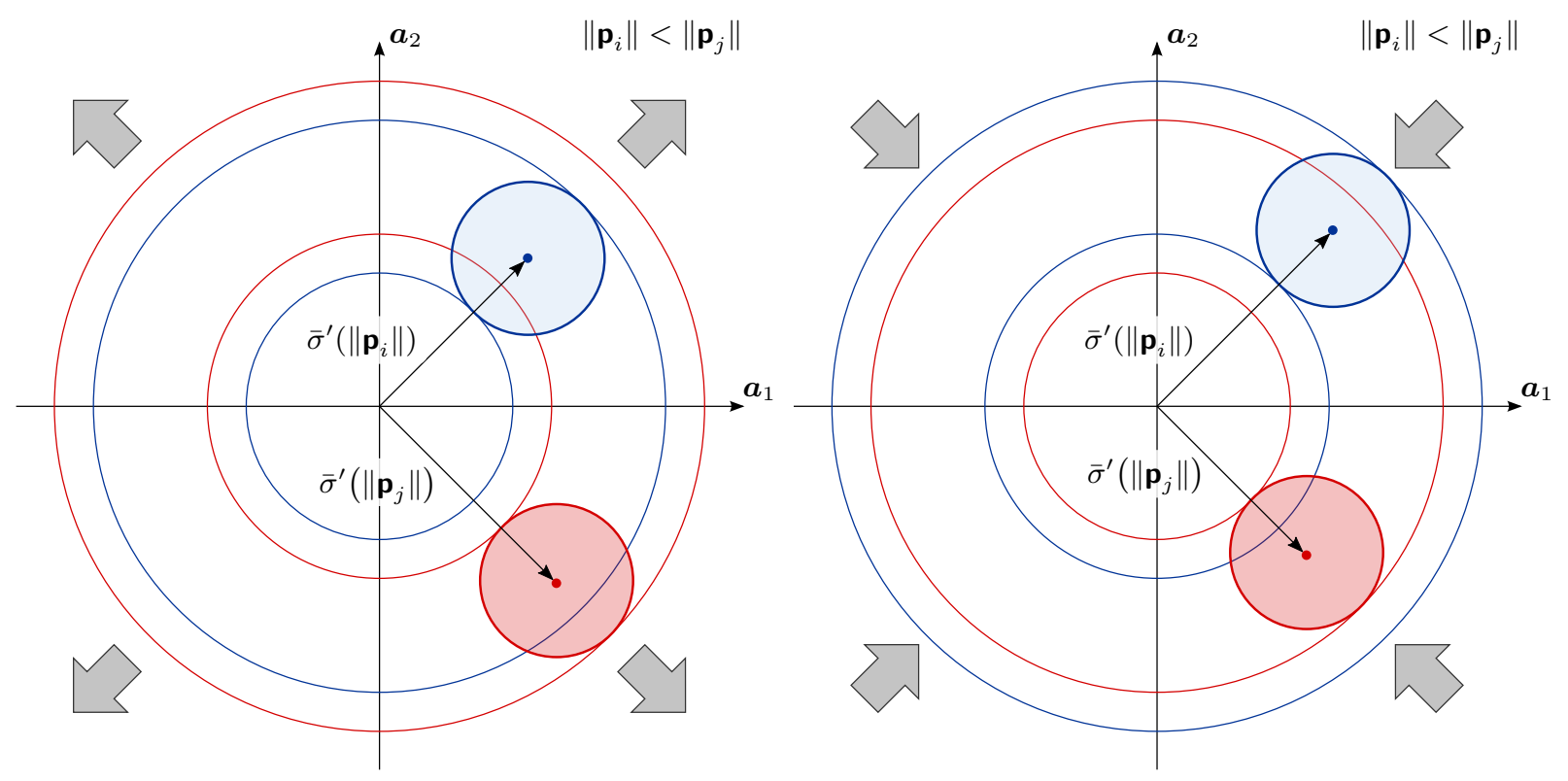

Figure 11: (left) Stress-hardening and (right) stress-softening behaviour of the transformation domain in the deviatoric space $(N=2)$ with constant $\mu$ and, respectively, $\bar{\sigma}^{\prime \prime}>0$ and $\bar{\sigma}^{\prime \prime}<0$.
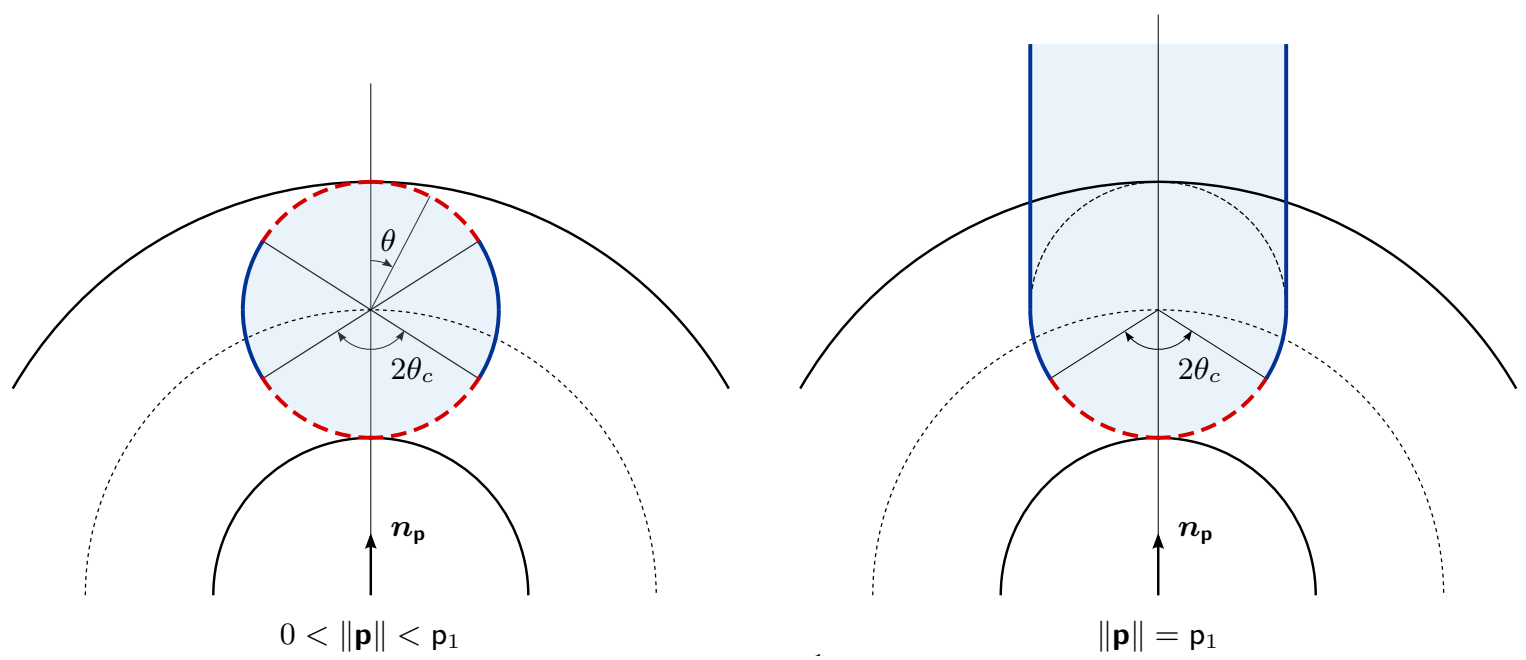

Figure 12: Stability results $(N=2)$ for stress-softening SMA model with constant $\mu$ and $\bar{\sigma}^{\prime \prime}<0$ in the deviatoric plane. The blue boundary lines denote stable states while the red-dashed boundary lines denote the unstable states. 


\section{Conclusions}

A three dimensional superelastic model for SMA has been derived in a consistent energetic framework. The isotropic model is defined from its strain energy density which is the sum of a free energy and a dissipated energy. Phase-transformation is assumed to be incompressible and is accounted through a unique internal tensorial variable $\mathbf{p}$. The model rely on two material functions of the norm of $\mathbf{p}$ as well as a material constant. As a result, we have departed from most simple hypothesis and main characteristic of SMA to build the constitutive behaviour of this three dimensional model. The richness of the model comes from the rigorous derivation of the evolution laws and second-order stability conditions in a general context where stress-softening and thus instabilities may occur.

Following previous works in fracture, damage, plasticity and more generally in the context of rateindependent materials, the quasi-static evolution problem of a domain is formulated compactly in terms of two physical principles written at the global level: a stability criterion and an energy balance condition. While the stability criterion selects the local minima of the total energy, the energy balance condition enforces the evolution of the total energy to be consistent with the work of external loading. First-order condition of stability leads to local phase transformation criteria with appropriate consistency conditions derived from the energy balance condition. Those phase transformation criteria define transformation domains, with clear geometric interpretation, in which the deviatoric stress must always belong. Instead, the consistency conditions gives the direction of rate $\dot{\mathbf{p}}$ according to a normality rule. It is worth noting that all these conditions are deduced and not postulated.

We have illustrated the numerical response of the model on a combined traction-torsion problem for a thin-walled cylinder for which homogeneous evolutions are investigated. The cylinder has been subjected to a circular strain loading path in order to highlight the main features of the three dimensional model. Indeed, we have observed that the transformation domain in the stress space moves accordingly to the transformation strain rate direction which, in turn, always follows the normality rule. These properties of the model are qualitatively in agreement with traction-torsion experimental results for SMA. Moreover, the analysis has taken into account the influence of stress-hardening and stress-softening behaviours. For instance it has been shown that stress-softening can lead to non-convex transformation domains in the stress space, in the common case of a shear modulus strongly dependent on the phase transformation strain.

Finally, we have studied the stability of homogeneous states which are classically used for identification

of the model parameters. As the study of first-order condition of stability are not sufficient to ensure local minimality of a phase-transforming homogeneous state, one has to focus on second-order conditions of stability which naturally arise in this energetic framework. The stability analysis has been performed in a multi-dimensional setting. While a stress-hardening model ensures stability of homogeneous states, a stresssoftening model might lead to macroscopic instabilities depending on the applied boundary conditions. These 
instabilities of homogeneous states tend to develop when proportional loading are considered. However, by considering specific non-proportional loading, we showed that homogeneous can be stable, hence allowing for a more consistent identification of the model. Necessary and sufficient conditions have been provided to compute such stabilizing loading directions and have been fully illustrated in a two-dimensional context.

For future works, regularization of the model to deal with macroscopic instabilities as well as a modification of the constitutive behaviour to capture the dissymmetry in traction and shear will be investigated. Those two issues should be tackled consistently in the current energetic framework by doing an appropriate modification of the density of strain energy. Evolution laws at the local level should be deduced as in this article directly from the stability criterion and energy balance condition.

\section{Acknowledgement}

The support provided by the "Istituto Nazionale di Alta Matematica Francesco Severi" (INdAM), through the grant "Progetto Giovani 2014 - GNFM", is gratefully acknowledged.

\section{Appendix 1: Proof of Proposition 2.1}

We have to prove an equivalence between the energetic formulation and the constitutive behavior written in terms of Kuhn-Tucker conditions.

Energetic formulation $10-(12) \Rightarrow$ Constitutive behaviour of the SMA (1) $-(6)$.

Proof. We assume that the energetic formulation 10-12 holds. We first prove the inequalities in the phase-transformation criteria (1)-(6). For a given state $(\varepsilon, p, \bar{p})$ with $|\mathrm{p}|<\mathrm{p}_{1}$, we consider a test direction $\mathrm{p}^{*}=\mathrm{p}+h \delta \mathrm{p}$ with $\delta \mathrm{p} \in \mathbb{R}$ and $0<h \ll 1$. Since the martensite strain is bounded, we have $\delta \mathrm{p} \leq 0$ if $\mathrm{p}=\mathrm{p}_{1}$ and $\delta \mathrm{p} \geq 0$ if $\mathrm{p}=-\mathrm{p}_{1}$. Expanding the total strain work around the state $(\varepsilon, \mathrm{p}, \overline{\mathrm{p}})$ up to the first order in $h$, we find

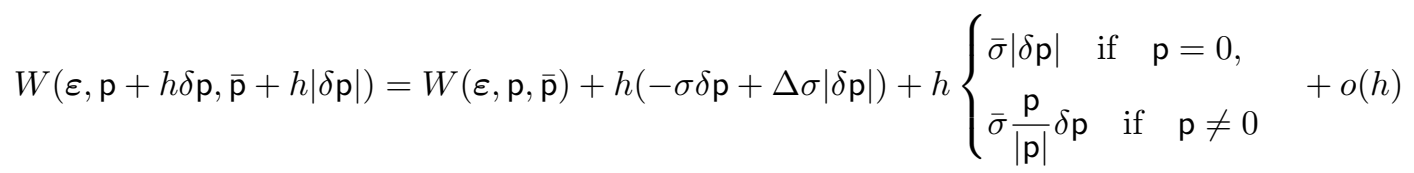

First, let us focus on the case $p=0$. In this case, invoking the material stability criterion (11), we have

$$
-\sigma \delta \mathrm{p}+\Delta \sigma|\delta \mathrm{p}|+\bar{\sigma}|\delta \mathrm{p}| \geq 0, \quad \forall \delta \mathrm{p}
$$

from which we deduce that

$$
|\sigma| \leq(\bar{\sigma}+\Delta \sigma)=\sigma_{\mathrm{Fw}}
$$


Now suppose that $p \neq 0$. Then, from the material stability criterion we have

$$
-\sigma \delta \mathrm{p}+\Delta \sigma|\delta \mathrm{p}|+\bar{\sigma} \frac{\mathrm{p}}{|\mathrm{p}|}|\delta \mathrm{p}| \geq 0, \quad \forall \delta \mathrm{p}
$$

from which we deduce

$$
\frac{\delta \mathrm{p}}{|\delta \mathrm{p}|}\left(\sigma-\frac{\mathrm{p}}{|\mathrm{p}|} \bar{\sigma}\right) \leq \Delta \sigma, \quad \forall \delta \mathrm{p} \neq 0
$$

Now, if $0 \leq p<p_{1}$, we get

$$
|\sigma-\bar{\sigma}| \leq \Delta \sigma \quad \Leftrightarrow \quad \sigma_{\mathrm{Rv}} \leq \sigma \leq \sigma_{\mathrm{Fw}}
$$

Conversely, if $-\mathrm{p}_{1}<\mathrm{p} \leq 0$, we get

$$
|\sigma+\bar{\sigma}| \leq \Delta \sigma \quad \Leftrightarrow \quad-\sigma_{\mathrm{Fw}} \leq \sigma \leq-\sigma_{\mathrm{Rv}}
$$

For the limiting case $\mathrm{p}=\mathrm{p}_{1}$ and $\mathrm{p}=-\mathrm{p}_{1}$, due to the imposed sign of the test direction $\delta \mathrm{p}$, we have

$$
\left\{\begin{array}{l}
\sigma-\bar{\sigma} \geq-\Delta \sigma \quad \Leftrightarrow \quad \sigma_{\mathrm{Rv}} \leq \sigma \quad \text { if } \quad \mathrm{p}=\mathrm{p}_{1}, \\
\sigma+\bar{\sigma} \geq \Delta \sigma \quad \Leftrightarrow \quad \sigma \leq-\sigma_{\mathrm{Rv}} \quad \text { if } \quad \mathrm{p}=-\mathrm{p}_{1} .
\end{array}\right.
$$

Thus, the material stability provide all the constitutive inequalities in any case that define the elastic domains of the SMA. To derive the consistency conditions of the constitutive behaviour, we now apply the energy balance condition. Taking the derivative of the total strain work (9) with respect to $t$, we have

$$
\dot{W}=\sigma \dot{\varepsilon}-\sigma \dot{\mathbf{p}}+\Delta \sigma|\dot{\mathbf{p}}|+\left\{\begin{array}{l}
\bar{\sigma}|\dot{\mathrm{p}}| \quad \text { if } \quad \mathrm{p}=0, \\
\bar{\sigma} \frac{\mathrm{p}}{|\mathrm{p}|} \dot{\mathbf{p}} \quad \text { if } \quad \mathrm{p} \neq 0 .
\end{array}\right.
$$

For $\mathrm{p}=0$, the energy balance condition gives $\sigma \dot{\mathrm{p}}=(\bar{\sigma}+\Delta \sigma)|\dot{\mathrm{p}}|$. Therefore, we deduce that for $|\sigma|<\bar{\sigma}+\Delta \sigma$, we have necessarily $\dot{\mathrm{p}}=0$ while for $\sigma=\bar{\sigma}+\Delta \sigma$ (resp. $\sigma=-\bar{\sigma}-\Delta \sigma)$, we have $\dot{\mathrm{p}} \geq 0$ (resp. $\dot{p} \leq 0)$. For $\mathrm{p} \neq 0$, the energy balance condition gives

$$
\sigma \dot{\mathrm{p}}=\left(\bar{\sigma} \frac{\mathrm{p}}{|\mathrm{p}|} \frac{\dot{\mathrm{p}}}{|\dot{\mathrm{p}}|}+\Delta \sigma\right)|\dot{\mathrm{p}}|
$$

and the procedure is the same than the case $p=0$ to obtain the expected consistency conditions in (3)(6).

Constitutive behaviour of the SMA (1)-(6) $\Rightarrow$ Energetic formulation $100-12$.

Proof. We assume now that the constitutive conditions [2]-(6) holds. A direct calculation shows that

$$
W\left(\varepsilon, \mathrm{p}^{*}, \overline{\mathrm{p}}+\left|\mathrm{p}^{*}-\mathrm{p}\right|\right)-W(\varepsilon, \mathrm{p}, \overline{\mathrm{p}})=\frac{1}{2} \mathrm{E}_{0}(\varepsilon-\mathrm{p})^{2}+\sigma\left(\mathrm{p}-\mathrm{p}^{*}\right)+\bar{\sigma}\left(\left|\mathrm{p}^{*}\right|-|\mathrm{p}|\right)+\Delta \sigma\left|\mathrm{p}^{*}-\mathrm{p}\right|
$$

The function $F: \mathrm{p}^{*} \mapsto W\left(\varepsilon, \mathrm{p}^{*}, \overline{\mathrm{p}}+\left|\mathrm{p}^{*}-\mathrm{p}\right|\right)-W(\varepsilon, \mathrm{p}, \overline{\mathrm{p}})$ is continuous on $\left[-\mathrm{p}_{1}, \mathrm{p}_{1}\right]$ and is differentiable on $\left[-\mathrm{p}_{1}, \mathrm{p}_{1}\right]$ except at $\mathrm{p}^{*}=0$ and $\mathrm{p}^{*}=\mathrm{p}$. Again, several cases have to be discriminated. Let us assume that 
$0<\mathrm{p}<\mathrm{p}_{1}$. If $\mathrm{p}^{*}>\mathrm{p}$, then $F^{\prime}\left(\mathrm{p}^{*}\right)=-\sigma+\bar{\sigma}+\Delta \sigma$ and $F^{\prime}\left(\mathrm{p}^{*}\right) \geq 0$ in virtue of (3). Otherwise if $\mathrm{p}^{*}<\mathrm{p}$ and $\mathrm{p}^{*}>0$, then $F^{\prime}\left(\mathrm{p}^{*}\right)=-\sigma+\bar{\sigma}-\Delta \sigma$ and again $F^{\prime}\left(\mathrm{p}^{*}\right) \leq 0$ in virtue of $(3)$. Finally, if $\mathrm{p}^{*}<\mathrm{p}$ and $\mathrm{p}^{*}<0$, then $F^{\prime}\left(\mathrm{p}^{*}\right)=-\sigma+\bar{\sigma}-\Delta \sigma$. Since in $\sigma \geq \bar{\sigma}-\Delta \sigma \geq-\bar{\sigma}-\Delta \sigma$, then we have $F^{\prime}\left(\mathrm{p}^{*}\right) \leq 0$. Thus, $\mathrm{p}^{*} \mapsto F\left(\mathrm{p}^{*}\right)$ is decreasing on $\left[-\mathrm{p}_{1}, \mathrm{p}\right]$ and increasing on $\left[\mathrm{p}, \mathrm{p}_{1}\right]$. Then $F(\mathrm{p})=\frac{1}{2} \mathrm{E}_{0}(\varepsilon-\mathrm{p})^{2} \geq 0$ is the global minimum of $F$ and we deduce that the material stability in the case $0<\mathrm{p}<\mathrm{p}_{1}$. We invite the reader to verify that the remaining cases lead to the same conclusions on the material stability by applying similar arguments.

The energy balance condition is derived from the consistency conditions of (2)-(66). For instance, in the case $0<\mathrm{p}<\mathrm{p}_{1}$, the chain rule gives $\dot{W}=\sigma \dot{\varepsilon}-\sigma \dot{\mathfrak{p}}+\bar{\sigma} \dot{\mathfrak{p}}+\Delta \sigma|\dot{\mathfrak{p}}|$. The consistency conditions (3) give $\dot{\mathrm{p}}(\sigma-\bar{\sigma})=\Delta \sigma|\dot{\mathrm{p}}|$ and thus, $\dot{W}=\sigma \dot{\varepsilon}$. Based on similar arguments, the remaining cases are shown to give the same result.

\section{Appendix 2: Values of constants $A, e$ and G}

To study the sign of such quadratic form, let us rewrite the second-order derivative as follows

$$
\begin{aligned}
\mathcal{E}^{\prime \prime}(\boldsymbol{u}, \mathbf{p}, \overline{\mathbf{p}})(\boldsymbol{\delta} \boldsymbol{u}, \boldsymbol{\delta} \mathbf{p},\|\boldsymbol{\delta} \mathbf{p}\|)= & \int_{\Omega_{0}}\left(2 \mu(0)\left\|\boldsymbol{\varepsilon}^{D}(\boldsymbol{\delta} \boldsymbol{u})-\boldsymbol{\delta} \mathbf{p}-\mathrm{s}^{\prime}(0) \boldsymbol{\sigma}^{D}\right\| \boldsymbol{\delta} \mathbf{p}\|\|^{2}+K(\operatorname{Tr}(\boldsymbol{\varepsilon}(\boldsymbol{\delta} \boldsymbol{u})))^{2}\right. \\
& \left.+\left(\bar{\sigma}^{\prime \prime}(0)-\frac{s^{\prime \prime}(0)}{2}\left\|\boldsymbol{\sigma}^{D}\right\|^{2}\right)\|\boldsymbol{\delta} \mathbf{p}\|^{2}\right) \mathrm{d} x \\
& +\int_{\Omega \backslash \Omega_{0}}\left(2 \mu(\|\mathbf{p}\|)\left\|\boldsymbol{\varepsilon}^{D}(\boldsymbol{\delta} \boldsymbol{u})-\boldsymbol{\delta} \mathbf{p}-\mathrm{s}^{\prime}(\|\mathbf{p}\|) \boldsymbol{\sigma}^{D} \frac{\mathbf{p} \cdot \boldsymbol{\delta} \mathbf{p}}{\|\mathbf{p}\|}\right\|^{2}+K(\operatorname{Tr}(\boldsymbol{\varepsilon}(\boldsymbol{\delta} \boldsymbol{u})))^{2}\right. \\
& +\left(\bar{\sigma}^{\prime}(\|\mathbf{p}\|)-\frac{\mathrm{s}^{\prime}(\|\mathbf{p}\|)}{2}\left\|\boldsymbol{\sigma}^{D}\right\|^{2}\right)\left(\frac{\|\boldsymbol{\delta} \mathbf{p}\|^{2}}{\|\mathbf{p}\|}-\frac{(\mathbf{p} \cdot \boldsymbol{\delta} \mathbf{p})^{2}}{\|\mathbf{p}\|^{3}}\right) \\
& \left.+\left(\bar{\sigma}^{\prime \prime}(\|\mathbf{p}\|)-\frac{s^{\prime \prime}(\|\mathbf{p}\|)}{2}\left\|\boldsymbol{\sigma}^{D}\right\|^{2}\right) \frac{(\mathbf{p} \cdot \boldsymbol{\delta} \mathbf{p})^{2}}{\|\mathbf{p}\|^{2}}\right) \mathrm{d} x
\end{aligned}
$$

with $\mathbf{s}(\|\mathbf{p}\|)=1 / 2 \mu(\|\mathbf{p}\|)$.

We give here the value of the constants $\boldsymbol{A}, \boldsymbol{e}$ and $\mathrm{G}$ involved in the Rayleigh ratio (71). Let us define $\boldsymbol{f}$ as

$$
\boldsymbol{f}=\left\{\begin{array}{l}
\boldsymbol{\sigma}^{D} \quad \text { if } \quad\|\mathbf{p}\|=0, \\
\boldsymbol{\sigma}^{D}-R\left(\|\mathbf{p}\|,\left\|\boldsymbol{\sigma}^{D}\right\|\right) \boldsymbol{n}_{\mathbf{p}} \quad \text { if } \quad 0<\|\mathbf{p}\|<1 \quad \text { or } \quad\|\mathbf{p}\|=\mathrm{p}_{1} \quad \text { with } \quad \mathbf{p} \cdot\left(\boldsymbol{\sigma}^{D}-R\left(\left\|\mathrm{p}_{1}\right\|,\left\|\boldsymbol{\sigma}^{D}\right\|\right) \boldsymbol{n}_{\mathbf{p}}\right)<0, \\
\operatorname{proj}_{\perp \mathbf{p}}\left(\boldsymbol{\sigma}^{D}-R\left(\mathbf{p}_{1},\left\|\boldsymbol{\sigma}^{D}\right\|\right) \boldsymbol{n}_{\mathbf{p}}\right) \quad \text { if } \quad\|\mathbf{p}\|=\mathrm{p}_{1} \quad \text { with } \quad \mathbf{p} \cdot\left(\boldsymbol{\sigma}^{D}-R\left(\left\|\mathrm{p}_{1}\right\|,\left\|\boldsymbol{\sigma}^{D}\right\|\right) \boldsymbol{n}_{\mathbf{p}}\right) \geq 0
\end{array}\right.
$$

Then, if $\|\mathbf{p}\|=0$, we have

$$
\left\{\begin{array}{l}
\boldsymbol{A} \varepsilon=2 \mu(0) \varepsilon^{D}+K(\operatorname{tr}(\boldsymbol{\varepsilon})) \mathbf{1} \\
\boldsymbol{e}=\boldsymbol{f}+\mathrm{s}^{\prime}(0)\|\boldsymbol{f}\| \boldsymbol{\sigma}^{D} \\
\mathrm{G}=\bar{\sigma}^{\prime \prime}(0)-\frac{1}{2} \mathrm{~s}^{\prime \prime}(0)\left(\left\|\boldsymbol{\sigma}^{D}\right\|\|\boldsymbol{f}\|\right)^{2}
\end{array}\right.
$$


while if $\|\mathbf{p}\|>0$, we have

$$
\left\{\begin{array}{l}
\boldsymbol{A} \boldsymbol{\varepsilon}=2 \mu(\|\mathbf{p}\|) \varepsilon^{D}+K(\operatorname{tr}(\boldsymbol{\varepsilon})) \mathbf{1} \\
\boldsymbol{e}=\boldsymbol{f}+\mathrm{s}^{\prime}(\|\mathbf{p}\|)\left(\boldsymbol{n}_{\mathbf{p}} \cdot \boldsymbol{f}\right) \boldsymbol{\sigma}^{D} \\
\mathrm{G}=\frac{1}{\|\mathbf{p}\|}\left(\bar{\sigma}^{\prime}(\|\mathbf{p}\|)-\frac{1}{2} \mathrm{~s}^{\prime}(\|\mathbf{p}\|)\left\|\boldsymbol{\sigma}^{D}\right\|^{2}\right)\left(\|\boldsymbol{f}\|^{2}-\left(\boldsymbol{n}_{\mathbf{p}} \cdot \boldsymbol{f}\right)^{2}\right)-\left(\bar{\sigma}^{\prime \prime}(\|\mathbf{p}\|)-\frac{1}{2} \mathrm{~s}^{\prime \prime}(\|\mathbf{p}\|)\left\|\boldsymbol{\sigma}^{D}\right\|^{2}\right)\left(\boldsymbol{n}_{\mathbf{p}} \cdot \boldsymbol{f}\right)^{2}
\end{array}\right.
$$

with $\mathbf{s}(\|\mathbf{p}\|)=1 / 2 \mu(\|\mathbf{p}\|)$.

[1] Alessi, R., Bernardini, D., Jun. 2015. Analysis of localization phenomena in Shape Memory Alloys bars by a variational approach. International Journal of Solids and Structures 7374, 113-133.

URL http://www.sciencedirect.com/science/article/pii/S0020768315002851

[2] Alessi, R., Marigo, J.-J., Vidoli, S., Jun. 2014. Gradient Damage Models Coupled with Plasticity and Nucleation of Cohesive Cracks. Archive for Rational Mechanics and Analysis 214 (2), 575-615.

URL http://link. springer .com/10.1007/s00205-014-0763-8

[3] Alessi, R., Marigo, J.-J., Vidoli, S., 2015. Gradient damage models coupled with plasticity: Variational formulation and main properties. Mechanics of Materials 80, Part B (0), 351-367.

URL http://www . sciencedirect .com/science/article/pii/S0167663614000039

[4] Auricchio, F., Mielke, A., Stefanelli, U., Nov. 2008. A rate-independent model for the isothermal quasistatic evolution of shape-memory materials. Mathematical Models and Methods in Applied Sciences 18 (125), 125-164.

URL http://www.worldscientific.com/doi/abs/10.1142/S0218202508002632

[5] Auricchio, F., Sacco, E., Nov. 1997. A one-dimensional model for superelastic shape-memory alloys with different elastic properties between austenite and martensite. International Journal of Non-Linear Mechanics 32 (6), 1101-1114.

URL http://www. sciencedirect.com/science/article/pii/S0020746296001308

[6] Bernardini, D., Pence, T. J., Dec. 2002. Models for one-variant shape memory materials based on dissipation functions. International Journal of Non-Linear Mechanics 37 (8), 1299-1317.

URL http://linkinghub.elsevier.com/retrieve/pii/S0020746202000203

[7] Bernardini, D., Pence, T. J., 2009. Mathematical Models for Shape Memory Materials. In: Schwartz, M. (Ed.), Smart Materials.

[8] Bourdin, B., Francfort, G. A., Marigo, J.-J., Mar. 2008. The Variational Approach to Fracture. Journal of Elasticity 91 (1-3), 5-148.

URL http://www . springerlink. com/content/31370771n83r2637 
[9] Bouvet, C., 2001. De L'uniaxial au multiaxial: comportement pseudoelastique des alliages a memoire de forme. Ph.D. thesis.

[10] Churchill, C. B., Shaw, J. A., Iadicola, M. A., Sep. 2009. Tips and tricks for characterizing shape memory alloy wire: Part 3-localization and propagation phenomena. Experimental Techniques 33 (5), $70-78$.

URL http://doi.wiley.com/10.1111/j.1747-1567.2009.00558.x

[11] Coleman, B. D., Gurtin, M. E., 1967. Thermodynamics with Internal State Variables. The Journal of Chemical Physics 47 (2), 597-613.

URL http://link . aip.org/link/?JCP/47/597/1

[12] Dal Maso, G., De Simone, A., Mora, M. G., 2006. Quasistatic Evolution Problems for Linearly Elasticity - Perfectly Plastic Materials. Archive for Rational Mechanics and Analysis 180 (2), 237-291.

URL http://dx.doi.org/10.1007/s00205-005-0407-0

[13] Echchorfi, R., Sep. 2013. Dialogue essais - simulation et identification de lois de comportement dalliage à mémoire de forme en chargement multiaxial. Ph.D. thesis.

URL http://www. theses.fr/2013ENAM0035

[14] Feng, P., Sun, Q.-P., Aug. 2006. Experimental investigation on macroscopic domain formation and evolution in polycrystalline NiTi microtubing under mechanical force. Journal of the Mechanics and Physics of Solids 54 (8), 1568-1603.

URL http://www.sciencedirect.com/science/article/pii/S0022509606000457

[15] Francfort, G. A., Marigo, J.-J., 1998. Revisiting brittle fracture as an energy minimization problem. Journal of the Mechanics and Physics of Solids 46 (8), 1319-1342.

URL

http://www.sciencedirect.com/science/article/B6TXB-3XDH3V4-2/2/ 6078bc5b38861a78a6b1044a60e0a4bb

[16] Gall, K., Jacobus, K., Sehitoglu, H., Maier, H. J., Mar. 1998. Stress-induced martensitic phase transformations in polycrystalline $\mathrm{CuZnAl}$ shape memory alloys under different stress states. Metallurgical and Materials Transactions A 29 (3), 765-773.

URL http://link.springer.com/10.1007/s11661-998-0267-y

[17] Hallai, J. F., Kyriakides, S., Aug. 2013. Underlying material response for Lüders-like instabilities. International Journal of Plasticity 47, 1-12.

URL http://www.sciencedirect.com/science/article/pii/S0749641912001854

[18] Halphen, B., Nguyen, Q. S., 1975. Generlazied Standard Materials. Journal de Mécanique 14 (1), 39-63. 
[19] Helm, D., Haupt, P., Jul. 2001. Thermomechanical Behaviour of Shape Memory Alloys. In: Lynch, C. S. (Ed.), SPIE's 8th Annual International Symposium on Smart Structures and Materials. International Society for Optics and Photonics, pp. 302-313.

URL http://proceedings.spiedigitallibrary.org/proceeding. aspx?articleid=908339

[20] Iadicola, M. A., Shaw, J. A., Feb. 2002. An Experimental Setup for Measuring Unstable Thermomechanical Behavior of Shape Memory Alloy Wire. Journal of Intelligent Materials Systems and Structures $13(2), 157-165$.

URL http://jim. sagepub.com/content/13/2-3/157.short

[21] Junker, P., Jaeger, S., Kastner, O., Eggeler, G., Hackl, K., Jul. 2015. Variational prediction of the mechanical behavior of shape memory alloys based on thermal experiments. Journal of the Mechanics and Physics of Solids 80, 86-102.

URL http://www.sciencedirect.com/science/article/pii/S0022509615000824

[22] Kohn, R. V., 1991. The relaxation of a double-well energy. Continuum Mechanics and Thermodynamics $3(3), 193-236$.

URL http://dx.doi.org/10.1007/BF01135336

[23] Lagoudas, D. C., Entchev, P. B., Popov, P., Patoor, E., Brinson, L. C., Gao, X., May 2006. Shape memory alloys, Part II: Modeling of polycrystals. Mechanics of Materials 38 (5-6), 430-462.

URL http://www.sciencedirect.com/science/article/pii/S0167663605001201

[24] León Baldelli, A. A., Maurini, C., Pham, K., Jan. 2015. A gradient approach for the macroscopic modeling of superelasticity in softening shape memory alloys. International Journal of Solids and Structures $52,45-55$.

URL http://www.sciencedirect.com/science/article/pii/S0020768314003564

[25] Lim, T. J., McDowell, D. L., Jan. 1999. Mechanical Behavior of an Ni-Ti Shape Memory Alloy Under Axial-Torsional Proportional and Nonproportional Loading. Journal of Engineering Materials and Technology 121 (1), 9.

URL http://materialstechnology . asmedigitalcollection . asme.org/article.aspx?articleid= 1425820

[26] Mielke, A., 2006. A Mathematical Framework for Generalized Standard Materials in the RateIndependent Case. In: Helmig, R., Mielke, A., Wohlmuth, B. (Eds.), Multifield Problems in Solid and Fluid Mechanics. Vol. 28 of Lecture Notes in Applied and Computational Mechanics. Springer Berlin / Heidelberg, pp. 399-428.

URL http://dx.doi .org/10.1007/978-3-540-34961-7_12 
[27] Mohd Jani, J., Leary, M., Subic, A., Gibson, M. A., Apr. 2014. A review of shape memory alloy research, applications and opportunities. Materials \& Design 56, 1078-1113.

URL http://www.sciencedirect.com/science/article/pii/S0261306913011345

[28] Moumni, Z., Zaki, W., Maitournam, H., 2009. Cyclic behavior and energy approach of the fatigue of Shape Memory Alloys. Journal of Mechanics of Materials and Structures 4 (February), 395-411.

[29] Patoor, E., Lagoudas, D. C., Entchev, P. B., Brinson, L. C., Gao, X., May 2006. Shape memory alloys, Part I: General properties and modeling of single crystals. Mechanics of Materials 38 (5-6), 391-429.

URL http://www.sciencedirect.com/science/article/pii/S0167663605001195

[30] Pham, K., 2014. An energetic formulation of a one-dimensional model of superelastic SMA. Continuum Mechanics and Thermodynamics 26 (6), 833-857.

[31] Pham, K., Marigo, J.-J., 2010. The variational approach to damage: I. The foundations. Comptes Rendus Mecanique 338 (4), 191-198.

[32] Pham, K., Marigo, J.-J., Apr. 2012. Stability of Homogeneous States with Gradient Damage Models: Size Effects and Shape Effects in the Three-Dimensional Setting. Journal of Elasticity 110 (1), 63-93.

URL http://link.springer.com/10.1007/s10659-012-9382-5http://dx.doi.org/10.1007/ s10659-012-9382-5

[33] Pham, K., Marigo, J.-J., Maurini, C., 2011. The issues of the uniqueness and the stability of the homogeneous response in uniaxial tests with gradient damage models. Journal of the Mechanics and Physics of Solids 59 (6), 1163-1190.

URL http://www.sciencedirect.com/science/article/B6TXB-52GXVJG-2/2/ 76efa9d53fe4f29eff9170a066d1302e

[34] Popov, P., Lagoudas, D. C., Oct. 2007. A 3-D constitutive model for shape memory alloys incorporating pseudoelasticity and detwinning of self-accommodated martensite. International Journal of Plasticity 23 (10-11), 1679-1720.

URL http://www.sciencedirect.com/science/article/pii/S0749641907000460

[35] Schwartz, M. (Ed.), 2008. Smart Materials. CRC.

[36] Shaw, J. A., Kyriakides, S., 1995. Thermomechanical aspects of NiTi. Journal of Mechanics Physics of Solids 43 (8), 1243-1281.

[37] Sittner, P., Hara, Y., Tokuda, M., Nov. 1995. Experimental study on the thermoelastic martensitic transformation in shape memory alloy polycrystal induced by combined external forces. Metallurgical 
and Materials Transactions A 26 (11), 2923-2935.

URL http://link.springer .com/10.1007/BF02669649

[38] Sittner, P., Takakura, M., Hara, Y., Tokuda, M., Jan. 1996. On Transformation Pathways of General Stress Controlled Thermoelastic Martensitic Transformation in Shape Memory Alloys. Le Journal de Physique IV 06 (C1), C1-357-C1-366.

URL http://dx.doi .org/10.1051/jp4:1996134

[39] Souza, A. C., Mamiya, E. N., Zouain, N., Sep. 1998. Three-dimensional model for solids undergoing stress-induced phase transformations. European Journal of Mechanics - A/Solids 17 (5), 789-806.

URL http://www.sciencedirect.com/science/article/pii/S0997753898800053

[40] Sun, Q.-P., Hwang, K. C., Jan. 1993. Micromechanics modelling for the constitutive behavior of polycrystalline shape memory alloysI. Derivation of general relations. Journal of the Mechanics and Physics of Solids 41 (1), 1-17.

URL http://www.sciencedirect.com/science/article/pii/002250969390060S

[41] Sun, Q.-P., Hwang, K. C., Jan. 1993. Micromechanics modelling for the constitutive behavior of polycrystalline shape memory alloysII. Study of the individual phenomena. Journal of the Mechanics and Physics of Solids 41 (1), 19-33.

URL http://www.sciencedirect.com/science/article/pii/002250969390061J

[42] Sun, Q.-P., Li, Z.-Q., Jun. 2002. Phase transformation in superelastic NiTi polycrystalline micro-tubes under tension and torsionfrom localization to homogeneous deformation. International Journal of Solids and Structures 39 (13-14), 3797-3809.

URL http://linkinghub.elsevier.com/retrieve/pii/S0020768302001828

[43] Wolfram Research, I., 2008. Mathematica 7.0.

[44] Zaki, W., Moumni, Z., Nov. 2007. A three-dimensional model of the thermomechanical behavior of shape memory alloys. Journal of the Mechanics and Physics of Solids 55 (11), 2455-2490.

URL http://www.sciencedirect.com/science/article/pii/S0022509607000749

[45] Ziólkowski, A., Oct. 2007. Three-dimensional phenomenological thermodynamic model of pseudoelasticity of shape memory alloys at finite strains. Continuum Mechanics and Thermodynamics 19 (6), 379-398.

URL http://link.springer.com/10.1007/s00161-007-0060-y 